\title{
A New Concept for the Separation of Anomeric Mixture of $\alpha / \beta$-D-Nucleosides through Regioselective Enzymatic Acylation or Hydrolysis Processes
}

\author{
Javier García, ${ }^{a}$ Alba Díaz-Rodríguez, ${ }^{\text {a }}$ Susana Fernández,, Yogesh S. Sanghvi, ${ }^{\text {b }}$ \\ Miguel Ferrero, ${ }^{a}$ and Vicente Gotor ${ }^{a, *}$
}
aDepartamento de Química Orgánica e Inorgánica and Instituto Universitario de Biotecnología de Asturias, Universidad de Oviedo, 33006-Oviedo (Asturias), Spain
${ }^{b}$ Rasayan Inc., 2802 Crystal Ridge Road, Encinitas, CA 92004-6615, USA

VGS@fq.uniovi.es

\section{SUPPORTING INFORMATION (page 1 of 79 pages)}

Table of Contents

\section{General Spectroscopic and Experimental Data (S3)}

\section{Experimental Procedures and Spectroscopical Data}

- General procedure for the regioselective enzymatic acylation of $\alpha-D-2$ 'deoxynucleosides 1 (S3)

- Spectroscopical Data of: 2a (S4), 2b (S4), 2c (S4), 2d (S4)

- Separation of an 1:1 anomeric mixture of $\alpha / \beta$-D-thymidine (S5)

- Spectroscopical Data of 4a (S5)

- Separation of an $1: 1$ anomeric mixture of $N^{6}$-benzoyl- $\alpha / \beta-D-2$ deoxyadenosine (S5)

- Spectroscopical Data of 4c (S5)

- 3',5'-di-O-levulinyl- $\alpha$-D-2'-deoxynucleosides 5 (S5)

- Spectroscopical Data of: 5a (S5), 5b (S6)

- General procedure for the enzymatic hydrolysis of 3',5'-di-O-levulinyl- $\alpha-D-2$ 'deoxynucleosides 5 (S6)

- Spectroscopical Data of: 6a (S6), 6b (S6)

- Separation of an 8:2 mixture of 3',5'-di-O-( $p$-chlorobenzoyl)- $\alpha / \beta$-D-thymidine from industrial synthesis of thymidine (S7)

- Spectroscopical Data of: 8 (S7), 9 (S7)

- General procedure for the enzymatic hydrolysis of 1-(3',5'-di-O-benzoyl-2'fluoro-2'-deoxy- $\alpha / \beta$-D-arabinofuranosyl)thymine (10/11) (S7)

- Spectroscopical Data of: 10:11 (S8), 12 (S8), 13 (S8)

- 1-(2'-Fluoro-2'-deoxy- $\alpha / \beta-D$-arabinofuranosyl)thymine (14/15) (S8)

- Enzymatic acylation of 1-(2'-fluoro-2'-deoxy- $\alpha / \beta$-D-arabinofuranosyl)thymine $(\mathbf{1 4} / \mathbf{1 5})(\mathrm{S} 9)$

- Spectroscopical Data of: 18 (S9), 19 (S9)

Copy of ${ }^{1} \mathrm{H}-,{ }^{13} \mathrm{C}$-, and DEPT-NMR Spectra of:

2a (S11-S13), 2b (S14-S16), 2c (S17-S19), 2d (S20-S22), 5a (S23-S25), 5b (S26-S28), 6a (S29-S31), 6b (S36-S38), 8 (S39-S41), 9 (S42-S44), 10:11 (S45S47), 12 (S52-S54), 13 (S60-S62), 14:15 (S68-S70), 18 (S72-S74), 19 (S76-S78). 
Copy of ${ }^{19} \mathrm{~F}$-NMR Spectra of:

10:11 (S48), 12 (S55), 13 (S63), 14:15 (S71), 18 (S75), 19 (S79).

Copy of 2D-NMR Spectra of 6a:

- HSQC $\left({ }^{1} \mathrm{~J}_{\mathrm{CH}}\right)(\mathrm{S} 32)$

- HMBC $\left({ }^{3} J_{\mathrm{CH}}\right)(\mathrm{S} 33)$

- COSY (S34)

- NOESY (S35)

Copy of 2D-NMR Spectra of 10:11:

- HSQC $\left({ }^{1} \mathrm{~J}_{\mathrm{CH}}\right)(\mathrm{S} 49)$

- HMBC $\left({ }^{3} J_{\mathrm{CH}}\right)(\mathrm{S} 50)$

- COSY (S51)

Copy of 2D-NMR Spectra of 12:

- HSQC $\left({ }^{1} J_{C H}\right)(S 56)$

- HMBC $\left({ }^{3} \mathrm{~J}_{\mathrm{CH}}\right)(\mathrm{S} 57)$

- COSY (S58)

- NOESY (S59)

Copy of 2D-NMR Spectra of 13:

- HSQC $\left({ }^{1} J_{\mathrm{CH}}\right)(\mathrm{S} 64)$

- HMBC $\left({ }^{3} J_{\mathrm{CH}}\right)(\mathrm{S} 65)$

- COSY (S66)

- NOESY (S67) 


\section{General Spectroscopic and Experimental Data}

General. Candida antarctica lipase B (CAL-B, Novozym 435, 10000 PLU/g) was purchased from Novo Nordisk. Pseudomonas cepacia lipase (PSL-C, $904 \mathrm{U} / \mathrm{g}$ ) was purchased from Amano Pharmaceuticals. Candida antarctica lipase A (CAL-A, $50 \mathrm{KU} / 19.24 \mathrm{~g})$ was purchased from Roche. Esterase BS2 (998 U/mL) was a gift from Julich. Pig liver esterase $(24 \mathrm{U} / \mathrm{mg})$ was purchased from Sigma Solvents were distilled over an adequate desiccant under nitrogen. HPLC analysis was performed at $254 \mathrm{~nm}$ using three methods: Method A: spherisorb W $5 \mu$ column $(250 \times 46 \mathrm{~mm}), 1.5 \mathrm{~mL} / \mathrm{min}$ flow, $25^{\circ} \mathrm{C}, \mathrm{CH}_{2} \mathrm{Cl}_{2} / \mathrm{PrOH}(9: 1)$ as eluent. Method $B$ : spherisorb ODS2 $5 \mu$ column $(250 \times 46 \mathrm{~mm}), 0.5 \mathrm{~mL} / \mathrm{min}$ flow, $25^{\circ} \mathrm{C}$, and two mobile phase (A: MeCN; B: $1.54 \mathrm{~g}$ ammonium acetate in $1000 \mathrm{~mL}$ water containing $1 \% \mathrm{MeCN})$. Gradient condition:

\begin{tabular}{ccc}
\hline Time $(\min )$ & A (\%) & B (\%) \\
\hline 0.0 & 1 & 99 \\
10.0 & 4 & 96 \\
15.0 & 4 & 96 \\
20.0 & 10 & 90 \\
25.0 & 20 & 80 \\
30.0 & 50 & 50 \\
40.0 & 70 & 30 \\
\hline
\end{tabular}

Method C: Waters X-Terra column $(250 \times 46 \mathrm{~mm}), 0.5 \mathrm{~mL} / \mathrm{min}$ flow, $25^{\circ} \mathrm{C}$, and two mobile phase (A: MeCN; B: $1.54 \mathrm{~g}$ ammonium acetate in $1000 \mathrm{~mL}$ water containing $1 \% \mathrm{MeCN})$. Gradient condition:

\begin{tabular}{ccc}
\hline Time $(\min )$ & A (\%) & B (\%) \\
\hline 0.0 & 30 & 70 \\
20.0 & 30 & 70 \\
20.1 & 50 & 50
\end{tabular}

Melting points were taken on samples in open capillary tubes and are uncorrected. IR spectra were recorded on an Infrared Fourier Transform spectrophotometer using $\mathrm{NaCl}$ plates or $\mathrm{KBr}$ pellets. Flash chromatography was performed using silica gel 60 (230-400 mesh). For routine experiments, ${ }^{1} \mathrm{H},{ }^{13} \mathrm{C}$ NMR, and DEPT were obtained using 200.13 or $300.13 \mathrm{MHz}$ for ${ }^{1} \mathrm{H}$, and 50.3 or $75.5 \mathrm{MHz}$ for ${ }^{13} \mathrm{C}$. An spectrometer operating at 400.13 and $100.61 \mathrm{MHz}$ for ${ }^{1} \mathrm{H}$ and ${ }^{13} \mathrm{C}$, respectively, was used for the acquisition of ${ }^{1} \mathrm{H}-{ }^{1} \mathrm{H}$ homonuclear (COSY and NOESY) and ${ }^{1} \mathrm{H}-{ }^{13} \mathrm{C}$ heteronuclear (HSQC and $\mathrm{HMBC}$ ) correlations. $\mathrm{ESI}^{+}$were used to record mass spectra (MS).

\section{Experimental Procedures and Spectroscopical Data}

General procedure for the regioselective enzymatic acylation of $\alpha-D-2$ 'deoxynucleosides 1 . In a standard procedure, THF $(2 \mathrm{~mL})$ was added to an Erlenmeyer flask that contained $1(0.2 \mathrm{mmol})$, O-levulinyl acetonoxime (102.6 $\mathrm{mg}$, $0.6 \mathrm{mmol}$ ), and the enzyme under nitrogen (the ratio of enzyme and time are indicated in Table 1). The reaction mixture was stirred at $30{ }^{\circ} \mathrm{C}$ and $250 \mathrm{rpm}$, and 
monitored by TLC $\left(10 \% \mathrm{MeOH} / \mathrm{CH}_{2} \mathrm{Cl}_{2}\right)$. The enzyme was filtered off, washed with $\mathrm{CH}_{2} \mathrm{Cl}_{2}$ and $\mathrm{MeOH}$, and the solvents evaporated under vacuum. Flash chromatography (EtOAc and then $20 \% \mathrm{MeOH} / \mathrm{CH}_{2} \mathrm{Cl}_{2}$ for $\mathbf{2 a}, \mathbf{2 b}$, and $\mathbf{2 c}$; gradient elution with $5-20 \% \mathrm{MeOH} / \mathrm{CH}_{2} \mathrm{Cl}_{2}$ for $2 \mathbf{d}$ ) of the residue afforded derivatives $\mathbf{2 a}-\mathbf{d}$ (yields are indicated in Table 1).

5'-O-Levulinyl- $\alpha$-D-thymidine (2a). $\underline{R}_{f}\left(10 \% \mathrm{MeOH} / \mathrm{CH}_{2} \mathrm{Cl}_{2}\right): 0.38 ; \mathrm{mp}: 87-89{ }^{\circ} \mathrm{C}$; $\underline{\text { IR }}(\mathrm{KBr}): \mathrm{v} \quad 3055,2987,1740,1706$, and $1700 \mathrm{~cm}^{-1} ;{ }^{1} \mathrm{H}$ NMR $\left(\mathrm{MeOH}-d_{4}, 200\right.$ $\mathrm{MHz}): \delta 2.08(\mathrm{~s}, 3 \mathrm{H}, \mathrm{Me}), 2.22-2.31\left(\mathrm{~m}, 1 \mathrm{H}, \mathrm{H}_{2^{\prime}}\right), 2.36(\mathrm{~s}, 3 \mathrm{H}, \mathrm{Me}-\mathrm{Lev}), 2.76(\mathrm{~m}$, $2 \mathrm{H}, \mathrm{CH}_{2}$-Lev), $2.97\left(\mathrm{~m}, 3 \mathrm{H}, \mathrm{CH}_{2}\right.$-Lev $\left.+\mathrm{H}_{2}\right), 4.32\left(\mathrm{~m}, 2 \mathrm{H}, \mathrm{H}_{5^{\prime}}\right), 4.52\left(\mathrm{~m}, 1 \mathrm{H}, \mathrm{H}_{4^{\prime}}\right), 4.62$ $\left(\mathrm{m}, 1 \mathrm{H}, \mathrm{H}_{3^{\prime}}\right), 6.40\left(\mathrm{dd}, 1 \mathrm{H}, \mathrm{H}_{1},{ }^{3} \mathrm{~J}_{\mathrm{HH}} 7.4,{ }^{3} \mathrm{~J}_{\mathrm{HH}} 3.0 \mathrm{~Hz}\right)$, and $7.96\left(\mathrm{~d}, 1 \mathrm{H}, \mathrm{H}_{6},{ }^{4} \mathrm{~J}_{\mathrm{HH}}|1.2|\right.$ $\mathrm{Hz}) ;{ }^{13} \mathrm{C} \mathrm{NMR}\left(\mathrm{MeOH}-d_{4}, 75.5 \mathrm{MHz}\right): \delta 12.6(\mathrm{Me}), 28.8\left(\mathrm{CH}_{2}\right.$-Lev), 29.7 (Me-Lev), 38.6, $41.2\left(\mathrm{CH}_{2}-\mathrm{Lev}+\mathrm{C}_{2^{\prime}}\right), 65.3\left(\mathrm{C}_{5^{\prime}}\right), 72.5\left(\mathrm{C}_{3^{\prime}}\right), 87.6,87.9\left(\mathrm{C}_{1^{\prime}}+\mathrm{C}_{4^{\prime}}\right), 110.9\left(\mathrm{C}_{5}\right)$, $138.8\left(\mathrm{C}_{6}\right), 152.4\left(\mathrm{C}_{2}\right), 166.5\left(\mathrm{C}_{4}\right), 174.1(\mathrm{C}=\mathrm{O})$, and $209.6(\mathrm{C}=\mathrm{O}) ; \mathrm{MS}\left(\mathrm{ESI}^{+}, \mathrm{m} / \mathrm{z}\right)$ : $363\left[(\mathrm{M}+\mathrm{Na})^{+}, 100 \%\right]$ and $379\left[(\mathrm{M}+\mathrm{K})^{+}, 60\right]$; Anal. Calcd. $(\%)$ for $\mathrm{C}_{15} \mathrm{H}_{20} \mathrm{~N}_{2} \mathrm{O}_{7}: \mathrm{C}$, 52.92; $\mathrm{H}, 5.93$; N, 8.23. Found: $\mathrm{C}, 52.8 ; \mathrm{H}, 5.8 ; \mathrm{N}, 8.3$.

$N^{4}$-Benzoyl-5'-O-levulinyl- $\alpha$-D-2'-deoxycytidine (2b). $\quad \underline{R}_{f}\left(10 \% \mathrm{MeOH} / \mathrm{CH}_{2} \mathrm{Cl}_{2}\right)$ : 0.40 ; mp: $130-132^{\circ} \mathrm{C}$; IR $(\mathrm{KBr}):$ v 1740,1706 , and $1663 \mathrm{~cm}^{-1} ;{ }^{1} \mathrm{H}$ NMR $(\mathrm{MeOH}-$ $\left.d_{4}, 300 \mathrm{MHz}\right): \delta 2.36(\mathrm{~m}, 3 \mathrm{H}, M e-L e v), 2.66\left(\mathrm{~m}, 1 \mathrm{H}, \mathrm{H}_{2^{\prime}}\right), 2.78\left(\mathrm{~m}, 2 \mathrm{H}, \mathrm{CH}_{2}-\mathrm{Lev}\right)$,

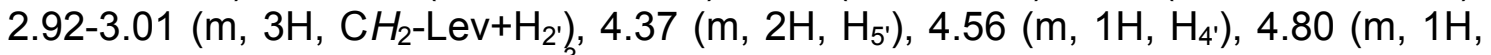
$\left.\mathrm{H}_{3^{\prime}}\right), 6.35$ (apparent d, $\left.1 \mathrm{H}, \mathrm{H}_{1^{\prime}},{ }^{3} \mathrm{~J}_{\mathrm{HH}} 6.9 \mathrm{~Hz}\right), 7.73\left(\mathrm{~m}, 4 \mathrm{H}, \mathrm{H}_{5}+\mathrm{H}_{\mathrm{m}}+\mathrm{H}_{\mathrm{p}}\right), 8.14(\mathrm{~m}, 2 \mathrm{H}$, $\mathrm{H}_{\mathrm{o}}$ ), and $8.46\left(\mathrm{~d}, 1 \mathrm{H}, \mathrm{H}_{6},{ }^{3} \mathrm{~J}_{\mathrm{HH}} 7.4 \mathrm{~Hz}\right) ; \stackrel{13}{\mathrm{C} ~ N M R}\left(\mathrm{MeOH}-d_{4}, 50.3 \mathrm{MHz}\right): \delta 28.7$ $\left(\mathrm{CH}_{2}\right.$-Lev), 29.7 (Me-Lev), 38.6, $41.8\left(\mathrm{CH}_{2}\right.$-Lev+C $\left.{ }^{\prime}\right), 65.2\left(\mathrm{C}_{5^{\prime}}\right), 72.6\left(\mathrm{C}_{3^{\prime}}\right), 88.8$, $90.7\left(\mathrm{C}_{1}, \mathrm{C}_{4^{\prime}}\right), 97.7\left(\mathrm{C}_{5}\right), 129.1,129.8\left(\mathrm{C}_{\mathrm{o}}+\mathrm{C}_{\mathrm{m}}\right), 134.0\left(\mathrm{C}_{\mathrm{p}}\right), 134.6\left(\mathrm{C}_{\mathrm{i}}\right), 146.8\left(\mathrm{C}_{6}\right)$, $157.7\left(\mathrm{C}_{2}\right), 164.5\left(\mathrm{C}_{4}\right), 168.8(\mathrm{PhC}=\mathrm{O}), 174.1(\mathrm{C}=\mathrm{O})$, and $209.4(\mathrm{C}=\mathrm{O}) ; \mathrm{MS}\left(\mathrm{ESI}^{+}\right.$, $\mathrm{m} / \mathrm{z}): 430\left[(\mathrm{M}+\mathrm{H})^{+}, 50 \%\right]$ and $452\left[(\mathrm{M}+\mathrm{Na})^{+}, 60\right] ;$ Anal. Calcd. (\%) for $\mathrm{C}_{21} \mathrm{H}_{23} \mathrm{~N}_{3} \mathrm{O}_{7}$ : C, 58.74; H, 5.40; N, 9.79. Found: C, 58.8; H, 5.5; N, 9.8.

$N^{6}$-Benzoyl-5'-O-levulinyl- $\alpha$-D-2'-deoxyadenosine (2c). $\underline{R}_{f}\left(10 \%{ }^{i} \mathrm{PrOH} / \mathrm{CH}_{2} \mathrm{Cl}_{2}\right)$ : 0.30; IR (KBr): v 2354, 1737, and $1713 \mathrm{~cm}^{-1} ;{ }^{1} \underline{\mathrm{H} N M R}\left(\mathrm{MeOH}-d_{4}, 300 \mathrm{MHz}\right): \delta$ $2.35\left(\mathrm{~s}, 3 \mathrm{H}\right.$, Me-Lev), $2.68\left(\mathrm{~m}, 1 \mathrm{H}, 1 \mathrm{H}_{2^{\prime}}\right), 2.77\left(\mathrm{~m}, 2 \mathrm{H}, \mathrm{CH}_{2}\right.$-Lev), $3.00\left(\mathrm{~m}, 2 \mathrm{H}, \mathrm{CH}_{2^{-}}\right.$ Lev), $3.09\left(\mathrm{~m}, 1 \mathrm{H}, 1 \mathrm{H}_{2^{\prime}}\right), 4.39\left(\mathrm{~m}, 2 \mathrm{H}, \mathrm{H}_{5^{\prime}}\right), 4.68\left(\mathrm{~m}, 2 \mathrm{H}, \mathrm{H}_{3^{\prime}}+\mathrm{H}_{4^{\prime}}\right), 6.78\left(\mathrm{dd}, 1 \mathrm{H}, \mathrm{H}_{1^{\prime}}\right.$, $\left.{ }^{3} J_{\mathrm{HH}} 7.7,2.6 \mathrm{~Hz}\right), 7.76\left(\mathrm{~m}, 3 \mathrm{H}, \mathrm{H}_{\mathrm{m}}+\mathrm{H}_{\mathrm{p}}\right), 8.26\left(\mathrm{~m}, 2 \mathrm{H}, \mathrm{H}_{\mathrm{o}}\right), 8.89\left(\mathrm{~s}, 1 \mathrm{H}, \mathrm{H}_{2}\right.$ or $\left.\mathrm{H}_{8}\right)$, and $8.92\left(\mathrm{~s}, 1 \mathrm{H}, \mathrm{H}_{8}\right.$ or $\left.\mathrm{H}_{2}\right), \stackrel{13}{\mathrm{C} ~ N M R}\left(\mathrm{MeOH}-d_{4}, 75.5 \mathrm{MHz}\right): \delta 28.8\left(\mathrm{CH}_{2}-\mathrm{Lev}\right), 29.7$ (Me-Lev), 38.6, $41.2\left(\mathrm{C}_{2^{\prime}}+\mathrm{CH}_{2}\right.$-Lev $), 65.2\left(\mathrm{C}_{5^{\prime}}\right), 72.9\left(\mathrm{C}_{3^{\prime}}\right)$, 86.6, $87.8\left(\mathrm{C}_{1^{\prime}}+\mathrm{C}_{4^{\prime}}\right), 124.9$ $\left(\mathrm{C}_{5}\right), 129.4,129.7\left(\mathrm{C}_{\mathrm{o}}+\mathrm{C}_{\mathrm{m}}\right), 133.9\left(\mathrm{C}_{\mathrm{p}}\right), 134.9\left(\mathrm{C}_{\mathrm{i}}\right), 144.9\left(\mathrm{C}_{8}\right), 150.9\left(\mathrm{C}_{6}\right), 152.9$, $153.0\left(\mathrm{C}_{2}+\mathrm{C}_{4}\right), 168.1(\mathrm{PhC}=\mathrm{O}), 174.2(\mathrm{C}=\mathrm{O})$, and $209.6(\mathrm{C}=\mathrm{O}) ; \quad \mathrm{MS}\left(\mathrm{ESI}^{+}, \mathrm{m} / \mathrm{z}\right)$ : $454\left[(\mathrm{M}+\mathrm{H})^{+}, 15 \%\right]$ and $476\left[(\mathrm{M}+\mathrm{Na})^{+}, 100\right]$; Anal. Calcd. (\%) for $\mathrm{C}_{22} \mathrm{H}_{23} \mathrm{~N}_{5} \mathrm{O}_{6}$ : C, 58.27; $\mathrm{H}, 5.11 ; \mathrm{N}, 15.44$. Found: $\mathrm{C}, 58.3 ; \mathrm{H}, 5.1 ; \mathrm{N}, 15.3$.

$N^{2}$-Isobutyryl-5'-O-levulinyl- $\alpha-D-2$ '-deoxyguanosine $\quad(2 d) . \quad \underline{R}_{f} \quad(15 \%$ $\left.\mathrm{MeOH} / \mathrm{CH}_{2} \mathrm{Cl}_{2}\right): 0.61$; IR $(\mathrm{NaCl}): \cup \quad 2370,1734$, and $1664 \mathrm{~cm}^{-1} ;{ }^{1} \underline{\mathrm{H}} \mathrm{NMR}(\mathrm{MeOH}-$ $\left.d_{4}, 200 \mathrm{MHz}\right): \delta 1.42\left(\mathrm{~d}, 6 \mathrm{H}, \mathrm{Me}-{ }^{\mathrm{i} B u},{ }^{3} \mathrm{~J}_{\mathrm{HH}} 7.1 \mathrm{~Hz}\right), 2.34$ (s, 3H, Me-Lev), 2.65-3.05 $\left(\mathrm{m}, 7 \mathrm{H}, 2 \mathrm{CH}_{2}-\mathrm{Lev}+\mathrm{H}_{2}, \mathrm{CH}-{ }^{\mathrm{i}} \mathrm{Bu}\right), 4.38\left(\mathrm{~m}, 2 \mathrm{H}, \mathrm{H}_{5^{\prime}}\right), 4.63\left(\mathrm{~m}, 2 \mathrm{H}, \mathrm{H}_{3^{\prime}}, \mathrm{H}_{4^{\prime}}\right), 6.51$ (dd, $\left.1 \mathrm{H}, \mathrm{H}_{1},{ }^{3} \mathrm{~J}_{\mathrm{HH}} 7.7,2.8 \mathrm{~Hz}\right)$, and $8.46\left(\mathrm{~s}, 1 \mathrm{H}, \mathrm{H}_{8}\right) ;{ }^{13} \mathrm{C} \mathrm{NMR}\left(\mathrm{MeOH}-d_{4}, 75.5 \mathrm{MHz}\right): \delta$ 19.2 (Me-'Bu), $28.7\left(\mathrm{CH}_{2}-\mathrm{Lev}\right), 29.6$ (Me-Lev), $36.9\left(\mathrm{CH}-{ }^{\mathrm{i}} \mathrm{Bu}\right), 38.5,40.8\left(\mathrm{C}_{2^{\prime}}+\mathrm{CH}_{2^{-}}\right.$ Lev), $65.1\left(\mathrm{C}_{5^{\prime}}\right), 72.6\left(\mathrm{C}_{3^{\prime}}\right), 86.0,86.9\left(\mathrm{C}_{1^{\prime}}+\mathrm{C}_{4^{\prime}}\right), 121.2\left(\mathrm{C}_{5}\right), 140.3\left(\mathrm{C}_{8}\right), 149.6,150.1$ $\left(\mathrm{C}_{2}+\mathrm{C}_{4}\right), 157.6\left(\mathrm{C}_{6}\right), 174.0(\mathrm{C}=\mathrm{O}), 181.6\left({ }^{\mathrm{i}} \mathrm{Bu}-\mathrm{C}=\mathrm{O}\right)$, and $209.4(\mathrm{C}=\mathrm{O}) ; \mathrm{MS}\left(\mathrm{ESI}^{+}\right.$, $\mathrm{m} / \mathrm{z}): 436\left[(\mathrm{M}+\mathrm{H})^{+}, 40 \%\right], 458\left[(\mathrm{M}+\mathrm{Na})^{+}, 30\right]$, and $474\left[(\mathrm{M}+\mathrm{K})^{+}, 42\right] ;$ Anal. Calcd. (\%) for $\mathrm{C}_{19} \mathrm{H}_{25} \mathrm{~N}_{5} \mathrm{O}_{7}: \mathrm{C}, 52.41 ; \mathrm{H}, 5.79 ; \mathrm{N}, 16.08$. Found: $\mathrm{C}, 52.3 ; \mathrm{H}, 5.7 ; \mathrm{N}, 16.2$. 
Separation of an 1:1 anomeric mixture of $\alpha / \beta$-D-thymidine. A similar procedure as that described for 2 was followed at $0.2 \mathrm{M}$ concentration. The reaction was monitored by HPLC (method A). Reaction time: $4 \mathrm{~h}$. Flash chromatography $\left(5 \%{ }^{i} \mathrm{PrOH} / \mathrm{CH}_{2} \mathrm{Cl}_{2}\right)$ of the reaction crude afforded $97 \%$ yield of 5'-O-levulinyl- $\alpha$-Dthymidine (2a) and $95 \%$ yield of 3'-O-levulinyl- $\beta$-D-thymidine (4a).

3'-O-Levulinyl- $\beta$-D-thymidine (4a). ${ }^{1}[\alpha]_{\mathrm{D}}^{20}=-3.0$ (c 0.8, $\left.\mathrm{CHCl}_{3}\right) ;{ }^{1} \underline{\mathrm{H}} \mathrm{NMR}(\mathrm{MeOH}-$ $d_{4}, 200 \mathrm{MHz}$ ): $\delta 2.09\left(\mathrm{~d}, 3 \mathrm{H}, \mathrm{Me},{ }^{4} \mathrm{~J}_{\mathrm{HH}}|1.3| \mathrm{Hz}\right), 2.39$ (s, 3H, Me-Lev), 2.57 (m, $\left.2 \mathrm{H}, \mathrm{H}_{2^{\prime}}\right), 2.80\left(\mathrm{t}, 2 \mathrm{H}, \mathrm{CH}_{2}\right.$-Lev, $\left.{ }^{3} \mathrm{~J}_{\mathrm{HH}} 6.1 \mathrm{~Hz}\right), 3.05\left(\mathrm{t}, 2 \mathrm{H}, \mathrm{CH}_{2}\right.$-Lev, $\left.{ }^{3} \mathrm{~J}_{\mathrm{HH}} 6.1 \mathrm{~Hz}\right), 4.01$ $\left(\mathrm{m}, 2 \mathrm{H}, \mathrm{H}_{5^{\prime}}\right), 4.29\left(\mathrm{~m}, 1 \mathrm{H}, \mathrm{H}_{4^{\prime}}\right), 5.02\left(\mathrm{~m}, 1 \mathrm{H}, \mathrm{H}_{3^{\prime}}\right), 6.50\left(\mathrm{dd}, 1 \mathrm{H}, \mathrm{H}_{1^{\prime}},{ }^{3} \mathrm{~J}_{\mathrm{HH}} 8.1,{ }^{3} \mathrm{~J}_{\mathrm{HH}} 6.5\right.$ $\mathrm{Hz})$, and $8.04\left(\mathrm{~d}, 1 \mathrm{H}, \mathrm{H}_{6},{ }^{4} \mathrm{~J}_{\mathrm{HH}}|1.3| \mathrm{Hz}\right)$.

Separation of an 1:1 anomeric mixture of $N^{6}$-benzoyl- $\alpha / \beta-D-2$ 'deoxyadenosine. A similar procedure as that described for 2 was followed at 0.2 $\mathrm{M}$ concentration. The reaction was monitored by HPLC (method A). Reaction time: $8 \mathrm{~h}$. Flash chromatography $\left(2 \%{ }^{i} \mathrm{PrOH} / \mathrm{CH}_{2} \mathrm{Cl}_{2}\right)$ of the crude afforded $91 \%$ yield of $N^{6}$-Benzoyl-5'-O-levulinyl- $\alpha$-D-2'-deoxyadenosine (2c) and $88 \%$ yield of $N^{6}$ Benzoyl-3'-O-levulinyl- $\beta-D-2$ '-deoxyadenosine (4c).

$\boldsymbol{N}^{6}$-Benzoyl-3'-O-levulinyl- $\beta$-D-2'-deoxyadenosine (4c). ${ }^{2} \quad[\alpha]_{\mathrm{D}}^{20}=-26.7$ (c 0.9 , $\left.\mathrm{CHCl}_{3}\right) ;{ }^{1} \underline{\mathrm{H} N M R}\left(\mathrm{CDCl}_{3}, 200 \mathrm{MHz}\right): \delta 2.22$ (s, 3H, Me-Lev), $2.61\left(\mathrm{~m}, 3 \mathrm{H}, \mathrm{CH}_{2}-\right.$ Lev $\left.+\mathrm{H}_{2^{\prime}}\right), 2.82\left(\mathrm{~m}, 2 \mathrm{H}, \mathrm{CH}_{2}\right.$-Lev), $3.18\left(\mathrm{~m}, 1 \mathrm{H}, \mathrm{H}_{2^{\prime}}\right), 3.97\left(\mathrm{~m}, 2 \mathrm{H}, \mathrm{H}_{5^{\prime}}\right), 4.31(\mathrm{~s}, 1 \mathrm{H}$, $\mathrm{H}_{4^{\prime}}$ ), 5.57 (apparent d, $1 \mathrm{H}, \mathrm{H}_{3^{\prime}},{ }^{3} \mathrm{~J}_{\mathrm{HH}} 5.4 \mathrm{~Hz}$ ), 6.38 (dd, $1 \mathrm{H}, \mathrm{H}_{1},{ }^{3} \mathrm{~J}_{\mathrm{HH}} 9.8,5.2 \mathrm{~Hz}$ ), $7.57\left(\mathrm{~m}, 3 \mathrm{H}, \mathrm{H}_{\mathrm{m}}+\mathrm{H}_{\mathrm{p}}\right), 8.05\left(\mathrm{~m}, 2 \mathrm{H}, \mathrm{H}_{\mathrm{o}}\right), 8.17\left(\mathrm{~s}, 1 \mathrm{H}, \mathrm{H}_{2}\right.$ or $\left.\mathrm{H}_{8}\right)$, and $8.79\left(\mathrm{~s}, 1 \mathrm{H}, \mathrm{H}_{8}\right.$ or $\mathrm{H}_{2}$ ).

3',5'-Di-O-levulinyl- $\alpha$-D-2'-deoxynucleosides 5. To a stirred mixture of $\mathbf{1}$ $(0.75 \mathrm{mmol})$ and $\mathrm{Et}_{3} \mathrm{~N}(0.3 \mathrm{~mL}, 1.9 \mathrm{mmol})$ in 1,4-dioxane $(5 \mathrm{~mL})$ under nitrogen, was added levulinic acid $(0.40 \mathrm{~mL}, 3.9 \mathrm{mmol}), \mathrm{DCC}(803.4 \mathrm{mg}, 3.9 \mathrm{mmol})$, and DMAP $(7.3 \mathrm{mg}, 0.06 \mathrm{mmol})$. The reaction was stirred at $\mathrm{rt}$ during $2.5 \mathrm{~h}$ for $1 \mathrm{a}$ and 40 min for 1b. The insoluble material was collected by filtration and the filtrate was evaporated under vacuum. The residue was taken up in $\mathrm{NaHCO}_{3}$ (aq) and extracted with $\mathrm{CH}_{2} \mathrm{Cl}_{2}$. The combined organic extracts were dried over $\mathrm{Na}_{2} \mathrm{SO}_{4}$ and evaporated. The residue was subjected to flash chromatography (EtOAc and then $5 \% \mathrm{MeOH} / \mathrm{CH}_{2} \mathrm{Cl}_{2}$ for $5 \mathbf{a}$; gradient elution with $0-30 \% \mathrm{MeOH} / \mathrm{EtOAc}$ for $5 \mathbf{b}$ ) to afford $\mathbf{5 a}$ in $93 \%$ yield and $\mathbf{5 b}$ in $94 \%$ yield.

3',5'-Di-O-levulinyl- $\alpha$-D-thymidine (5a). $\underline{R}_{f}\left(10 \% \mathrm{MeOH} / \mathrm{CH}_{2} \mathrm{Cl}_{2}\right): 0.40$; $\underline{\mathrm{IR}}(\mathrm{NaCl})$ : v 2926, 1742, and $1701 \mathrm{~cm}^{-1}$; ${ }^{1} \mathrm{H}$ NMR $\left(\mathrm{CDCl}_{3}, 300 \mathrm{MHz}\right): \delta 1.84(\mathrm{~s}, 3 \mathrm{H}, \mathrm{Me})$, 2.10 (s, 3H, Me-Lev), 2.12 (s, 3H, Me-Lev), $2.36\left(\mathrm{~m}, 1 \mathrm{H}, \mathrm{H}_{2^{\prime}}\right), 2.53-2.71(\mathrm{~m}, 9 \mathrm{H}$, $4 \mathrm{CH}_{2}$-Lev $\left.+\mathrm{H}_{2^{\prime}}\right), 4.15\left(\mathrm{~m}, 2 \mathrm{H}, \mathrm{H}_{5^{\prime}}\right), 4.58\left(\mathrm{~m}, 1 \mathrm{H}, \mathrm{H}_{4^{\prime}}\right), 5.13$ (apparent $\mathrm{d}, 1 \mathrm{H}, \mathrm{H}_{3^{\prime}},{ }^{3} \mathrm{~J}_{\mathrm{HH}}$ $5.7 \mathrm{~Hz}$ ), 6.23 (apparent d, $\left.1 \mathrm{H}, \mathrm{H}_{1},{ }^{3} \mathrm{~J}_{\mathrm{HH}} 7.1 \mathrm{~Hz}\right), 7.40\left(\mathrm{~s}, 1 \mathrm{H}, \mathrm{H}_{6}\right)$, and 9.91 (br.s, $1 \mathrm{H}$, $\mathrm{NH}) ; \stackrel{\underline{13}}{\mathrm{C} N M R}\left(\mathrm{CDCl}_{3}, 75.5 \mathrm{MHz}\right): \delta 12.2(\mathrm{Me}), 27.5\left(\mathrm{CH}_{2}-\mathrm{Lev}\right), 27.5\left(\mathrm{CH}_{2}\right.$-Lev $)$, 29.3 (Me-Lev), 29.5 (Me-Lev), 37.5, 37.7, $37.9\left(2 \mathrm{CH}_{2}-\mathrm{Lev}+\mathrm{C}_{2^{\prime}}\right), 63.7\left(\mathrm{C}_{5^{\prime}}\right), 74.5$ $\left(\mathrm{C}_{3^{\prime}}\right), 84.2,86.9\left(\mathrm{C}_{1^{\prime}}+\mathrm{C}_{4^{\prime}}\right), 109.7\left(\mathrm{C}_{5}\right), 135.5\left(\mathrm{C}_{6}\right), 150.3\left(\mathrm{C}_{2}\right), 164.2\left(\mathrm{C}_{4}\right), 172.0$

\footnotetext{
${ }^{1}$ previously reported in García, J.; Fernández, S.; Ferrero. M.; Sanghvi, Y. S.; Gotor, V. J. Org. Chem. 2002, 67, 4513-4519.

2 previously reported in García, J.; Fernández, S.; Ferrero. M.; Sanghvi, Y. S.; Gotor, V.

Tetrahedron: Asymmetry 2003, 14, 3197-3201.
} 
$(\mathrm{C}=\mathrm{O}), 206.3(\mathrm{C}=\mathrm{O})$, and $206.4(\mathrm{C}=\mathrm{O}) ; \quad \mathrm{MS}\left(\mathrm{ESI}^{+}, \mathrm{m} / \mathrm{z}\right): 439\left[(\mathrm{M}+\mathrm{H})^{+}, 20 \%\right]$ and $461\left[(\mathrm{M}+\mathrm{Na})^{+}, 80\right]$; Anal. Calcd. (\%) for $\mathrm{C}_{20} \mathrm{H}_{26} \mathrm{~N}_{2} \mathrm{O}_{9}$ : C, 54.77; $\mathrm{H}, 5.98 ; \mathrm{N}, 6.39$. Found: C, 54.9; H, 5.9; N, 6.5.

$N^{4}$-Benzoyl-3',5'-di-O-levulinyl- $\alpha$-D-2'-deoxycytidine $\quad$ (5b). $\quad \underline{R}_{f} \quad(10 \%$ $\mathrm{MeOH} / \mathrm{CH}_{2} \mathrm{Cl}_{2}$ ): 0.60; $\mathrm{mp}: 98-100^{\circ} \mathrm{C}$; IR (NaCl): v 3054, 2988, 1743, and 1717 $\mathrm{cm}^{-1} ;{ }^{1} \mathrm{H}$ NMR $\left(\mathrm{CDCl}_{3}, 300 \mathrm{MHz}\right): \delta 2.12$ (s, 3H, Me-Lev), 2.14 (s, 3H, Me-Lev), $2.28\left(\mathrm{~m}, 1 \mathrm{H}, \mathrm{H}_{2^{\prime}}\right), 2.55-2.85\left(\mathrm{~m}, 9 \mathrm{H}, \mathrm{CH}_{2}\right.$-Lev $\left.+\mathrm{H}_{2}\right), 4.22\left(\mathrm{~m}, 2 \mathrm{H}, \mathrm{H}_{5^{\prime}}\right), 4.70$ (apparent $\mathrm{t}, 1 \mathrm{H}, \mathrm{H}_{4},{ }^{3} \mathrm{~J}_{\mathrm{HH}} 4.0 \mathrm{~Hz}$ ), $5.15\left(\mathrm{~m}, 1 \mathrm{H}, \mathrm{H}_{3^{\prime}}\right), 6.22$ (apparent d, $1 \mathrm{H}, \mathrm{H}_{1^{\prime}},{ }^{3} J_{\mathrm{HH}} 6.6 \mathrm{~Hz}$ ), 7.44-7.55 (m, 4H, $\left.\mathrm{H}_{5}+\mathrm{H}_{\mathrm{m}}+\mathrm{H}_{\mathrm{p}}\right), 7.88\left(\mathrm{~m}, 2 \mathrm{H}, \mathrm{H}_{\mathrm{o}}\right)$, and $8.09\left(\mathrm{~d}, 1 \mathrm{H}, \mathrm{H}_{6},{ }^{3} \mathrm{~J}_{\mathrm{HH}} 7.4 \mathrm{~Hz}\right)$; ${ }^{13} \mathrm{C} \mathrm{NMR}\left(\mathrm{CDCl}_{3}, 75.5 \mathrm{MHz}\right): \delta 27.5\left(\mathrm{CH}_{2}\right.$-Lev $), 27.7\left(\mathrm{CH}_{2}\right.$-Lev), 29.4 (Me-Lev), 29.6 (Me-Lev), 37.6, 37.9, 38.1 $\left(\mathrm{C}_{2}+2 \mathrm{CH}_{2}\right.$-Lev), $63.6\left(\mathrm{C}_{5^{\prime}}\right), 74.6\left(\mathrm{C}_{3^{\prime}}\right), 85.3,89.3$ $\left(\mathrm{C}_{1},+\mathrm{C}_{4^{\prime}}\right), 95.5\left(\mathrm{C}_{5}\right), 127.6,128.6\left(\mathrm{C}_{\mathrm{o}}+\mathrm{C}_{\mathrm{m}}\right), 132.8\left(\mathrm{C}_{\mathrm{i}}\right), 132.9\left(\mathrm{C}_{\mathrm{p}}\right), 144.7\left(\mathrm{C}_{6}\right), 154.6$ $\left(\mathrm{C}_{2}\right), 162.5\left(\mathrm{C}_{4}\right), 166.7(\mathrm{PhC}=\mathrm{O}), 172.1(\mathrm{C}=\mathrm{O}), 172.1(\mathrm{C}=\mathrm{O}), 206.2(\mathrm{C}=\mathrm{O})$, and $206.5(\mathrm{C}=\mathrm{O}) ; \quad \mathrm{MS}\left(\mathrm{ESI}^{+}, \mathrm{m} / \mathrm{z}\right): 528\left[(\mathrm{M}+\mathrm{H})^{+}, 90 \%\right]$ and $550\left[(\mathrm{M}+\mathrm{Na})^{+}, 100\right] ;$ Anal. Calcd. (\%) for $\mathrm{C}_{26} \mathrm{H}_{29} \mathrm{~N}_{3} \mathrm{O}_{9}$ : C, 59.18; $\mathrm{H}, 5.54 ; \mathrm{N}, 7.97$. Found: $\mathrm{C}, 59.2 ; \mathrm{H}, 5.6 ; \mathrm{N}$, 7.9 .

General procedure for the enzymatic hydrolysis of 3',5'-di-O-levulinyl- $\alpha$ D-2'-deoxynucleosides $\mathbf{5}$. To a solution of $5(0.2 \mathrm{mmol})$ in 1,4-dioxane $(0.35 \mathrm{~mL})$ was added $0.15 \mathrm{M}$ phosphate buffer $\mathrm{pH} 7(1.65 \mathrm{~mL})$ and the corresponding lipase (the ratio of enzyme and time are indicated in Table 2). The reaction mixture was stirred at $30{ }^{\circ} \mathrm{C}$ and $250 \mathrm{rpm}$, and monitored by TLC $\left(10 \% \mathrm{MeOH} / \mathrm{CH}_{2} \mathrm{Cl}_{2}\right)$ and HPLC (Method B). The enzyme was filtered off, washed with $\mathrm{CH}_{2} \mathrm{Cl}_{2}$ and $\mathrm{MeOH}$, and the solvents evaporated under vacuum. For $\mathbf{6 b}$ the residue was taken up in $\mathrm{NaHCO}_{3}(\mathrm{aq})$ and extracted with $\mathrm{CH}_{2} \mathrm{Cl}_{2}$. The combined organic layers were dried over $\mathrm{Na}_{2} \mathrm{SO}_{4}$ and concentrated to give pure $6 \mathbf{b}$ in $96 \%$ yield (entry 3 , Table 2). For $6 a$ (entry 1 , Table 2) the residue was subjected to flash chromatography $(10 \%$ $\mathrm{MeOH} / \mathrm{CH}_{2} \mathrm{Cl}_{2}$ ) to afford $\mathbf{6 a}$ in $91 \%$ yield. For $\mathbf{6 b}$ (entry 4 , Table 2) the residue was subjected to flash chromatography $\left(5 \% \mathrm{MeOH} / \mathrm{CH}_{2} \mathrm{Cl}_{2}\right)$ to give pure $\mathbf{6 b}$ in $80 \%$ yield.

3'-O-levulinyl- $\alpha$-D-thymidine (6a). $\underline{R}_{f}\left(10 \% \mathrm{MeOH} / \mathrm{CH}_{2} \mathrm{Cl}_{2}\right): 0.35 ; \underline{\mathrm{IR}}(\mathrm{NaCl}): v$ 3455,1736 , and $1710 \mathrm{~cm}^{-1} ;{ }^{1} \underline{\mathrm{H} N M R}\left(\mathrm{CDCl}_{3}, 300 \mathrm{MHz}\right): \delta 1.89(\mathrm{~s}, 3 \mathrm{H}, \mathrm{Me}), 2.15$ (s, 3H, Me-Lev), 2.22 (s, 1H, $\mathrm{H}_{2^{\prime}}$ ), 2.40 (m, 2H, CH $\left.\mathrm{CH}_{2} \mathrm{Lev}\right), 2.78\left(\mathrm{~m}, 3 \mathrm{H}, \mathrm{CH}_{2^{-}}\right.$ Lev $\left.+\mathrm{H}_{2^{\prime}}\right), 3.73\left(\mathrm{~m}, 2 \mathrm{H}, \mathrm{H}_{5^{\prime}}\right), 4.47\left(\mathrm{~m}, 1 \mathrm{H}, \mathrm{H}_{4^{\prime}}\right), 5.25$ (apparent $\mathrm{d}, 1 \mathrm{H}, \mathrm{H}_{3^{\prime}},{ }^{3} \mathrm{~J}_{\mathrm{HH}} 6.3$ $\mathrm{Hz}$ ), 6.32 (apparent d, $\left.1 \mathrm{H}, \mathrm{H}_{1},{ }^{3} \mathrm{~J}_{\mathrm{HH}} 5.7 \mathrm{~Hz}\right)$, and $7.46\left(\mathrm{~s}, 1 \mathrm{H}, \mathrm{H}_{6}\right) ; \stackrel{13}{{ }_{\mathrm{C} \mathrm{NMR}}}\left(\mathrm{CDCl}_{3}\right.$, $75.5 \mathrm{MHz}): \delta 12.4(\mathrm{Me}), 27.9\left(\mathrm{CH}_{2}\right.$-Lev), 29.5 (Me-Lev), 37.9, 38.4 $\left(\mathrm{CH}_{2}\right.$-Lev+C $\left.2_{2^{\prime}}\right)$, $62.7\left(\mathrm{C}_{5^{\prime}}\right), 75.2\left(\mathrm{C}_{3^{\prime}}\right), 86.9\left(\mathrm{C}_{4^{\prime}}\right), 87.5\left(\mathrm{C}_{1}\right), 110.0\left(\mathrm{C}_{5}\right), 136.0\left(\mathrm{C}_{6}\right), 150.7\left(\mathrm{C}_{2}\right), 164.3$ $\left(\mathrm{C}_{4}\right), 172.4(\mathrm{C}=\mathrm{O})$, and $206.6(\mathrm{C}=\mathrm{O}) ; \mathrm{MS}\left(\mathrm{ESI}^{+}, \mathrm{m} / \mathrm{z}\right): 341\left[(\mathrm{M}+\mathrm{H})^{+}, 100 \%\right]$ and 363 $\left[(\mathrm{M}+\mathrm{Na})^{+}, 80\right]$; Anal. Calcd. (\%) for $\mathrm{C}_{15} \mathrm{H}_{20} \mathrm{~N}_{2} \mathrm{O}_{7}: \mathrm{C}, 52.92 ; \mathrm{H}, 5.93 ; \mathrm{N}, 8.23$. Found: C, $52.7 ; \mathrm{H}, 6.1 ; \mathrm{N}, 8.4$.

$N^{4}$-Benzoyl-3'-O-levulinyl- $\alpha$-D-2'-deoxycytidine (6b). $\quad R_{f}\left(10 \% \mathrm{MeOH} / \mathrm{CH}_{2} \mathrm{Cl}_{2}\right)$ : 0.28; IR (NaCl): v 3055, 2984, 1716, and $1651 \mathrm{~cm}^{-1} ;{ }^{1} \underline{\mathrm{H}} \mathrm{NMR}\left(\mathrm{MeOH}-d_{4}, 300\right.$ $\mathrm{MHz}): \delta 2.29\left(\mathrm{~s}, 3 \mathrm{H}\right.$, Me-Lev), $2.40\left(\mathrm{~m}, 1 \mathrm{H}, \mathrm{H}_{2^{\prime}}\right), 2.52\left(\mathrm{~m}, 2 \mathrm{H}, \mathrm{CH}_{2}\right.$-Lev), $2.90(\mathrm{~m}$, $2 \mathrm{H}, \mathrm{CH}_{2}$-Lev), $3.02\left(\mathrm{~m}, 1 \mathrm{H}, \mathrm{H}_{2^{\prime}}\right), 3.90\left(\mathrm{~m}, 2 \mathrm{H}, \mathrm{H}_{5^{\prime}}\right), 4.82\left(\mathrm{~m}, 1 \mathrm{H}, \mathrm{H}_{4^{\prime}}\right), 5.43$ (apparent $\left.\mathrm{d}, 1 \mathrm{H}, \mathrm{H}_{3},{ }^{3} \mathrm{~J}_{\mathrm{HH}} 5.7 \mathrm{~Hz}\right), 6.39$ (apparent d, $\left.1 \mathrm{H}, \mathrm{H}_{1},{ }^{3} \mathrm{~J}_{\mathrm{HH}} 6.5 \mathrm{~Hz}\right), 7.70-7.80(\mathrm{~m}, 4 \mathrm{H}$, $\left.\mathrm{H}_{5}+\mathrm{H}_{\mathrm{m}}+\mathrm{H}_{\mathrm{p}}\right), 8.13\left(\mathrm{~m}, 2 \mathrm{H}, \mathrm{H}_{\mathrm{o}}\right)$, and $8.46\left(\mathrm{~d}, 1 \mathrm{H}, \mathrm{H}_{6},{ }^{3} \mathrm{~J}_{\mathrm{HH}} 7.7 \mathrm{~Hz}\right) ; \stackrel{13}{\mathrm{C} N M R}(\mathrm{MeOH}-$ $\left.d_{4}, 75.5 \mathrm{MHz}\right): \delta 29.0\left(\mathrm{CH}_{2}\right.$-Lev), 29.6 (Me-Lev), 38.7, $39.9\left(\mathrm{CH}_{2}-\mathrm{Lev}+\mathrm{C}_{2^{\prime}}\right), 63.3$ $\left(\mathrm{C}_{5^{\prime}}\right), 76.4\left(\mathrm{C}_{3^{\prime}}\right), 90.2,91.1\left(\mathrm{C}_{1^{\prime}}+\mathrm{C}_{4^{\prime}}\right), 97.6\left(\mathrm{C}_{5}\right), 129.1,129.8\left(\mathrm{C}_{\mathrm{o}}+\mathrm{C}_{\mathrm{m}}\right), 134.1\left(\mathrm{C}_{\mathrm{p}}\right)$, 
$134.6\left(\mathrm{C}_{\mathrm{i}}\right), 146.4\left(\mathrm{C}_{6}\right), 157.7\left(\mathrm{C}_{2}\right), 164.8\left(\mathrm{C}_{4}\right), 169.0(\mathrm{PhC}=\mathrm{O}), 173.8(\mathrm{C}=\mathrm{O})$, and $209.4(\mathrm{C}=\mathrm{O}) ; \mathrm{MS}\left(\mathrm{ESI}^{+}, \mathrm{m} / \mathrm{z}\right): 430\left[(\mathrm{M}+\mathrm{H})^{+}, 30 \%\right]$ and $452\left[(\mathrm{M}+\mathrm{Na})^{+}, 100\right] ;$ Anal. Calcd. (\%) for $\mathrm{C}_{21} \mathrm{H}_{23} \mathrm{~N}_{3} \mathrm{O}_{7}$ : C, 58.74; $\mathrm{H}, 5.40 ; \mathrm{N}, 9.79$. Found: $\mathrm{C}, 58.7 ; \mathrm{H}, 5.5 ; \mathrm{N}$, 9.9 .

Separation of an 8:2 mixture of $3^{\prime}, 5^{\prime}-d i-O-(p$-chlorobenzoyl)- $\alpha / \beta$-Dthymidine from industrial synthesis of thymidine. To a solution of $7 / 8$ (52 $\mathrm{mg}$, $0.1 \mathrm{mmol})$ in 1,4-dioxane $(0.18 \mathrm{~mL})$ was added $0.15 \mathrm{M}$ phosphate buffer $\mathrm{pH} 7$ (0.83 $\mathrm{mL})$ and PSL-C (156 mg) or CAL-B $(52 \mathrm{mg})$. The mixture was stirred at $250 \mathrm{rpm}$ and $60{ }^{\circ} \mathrm{C}$ during $164 \mathrm{~h}$ or $104 \mathrm{~h}$, respectively. The enzyme was filtered off, washed with $\mathrm{CH}_{2} \mathrm{Cl}_{2}$ and $\mathrm{MeOH}$, and the solvents were evaporated under vacuum. Flash chromatography $\left(0.5 \%{ }^{i} \mathrm{PrOH} / \mathrm{CH}_{2} \mathrm{Cl}_{2}\right)$ give 8 in $80-84 \%$ yield and 9 in $67-71 \%$ yield.

3',5'-Di-O-(p-chlorobenzoyl)- $\beta$-D-thymidine (8). $\underline{R}_{f}\left(10 \%{ }^{i} \mathrm{PrOH} / \mathrm{CH}_{2} \mathrm{Cl}_{2}\right): 0.51$; $\underline{\mathrm{IR}}$

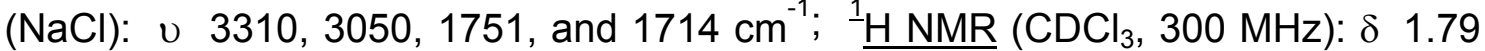
(s, 3H, Me), $2.32\left(\mathrm{~m}, 1 \mathrm{H}, \mathrm{H}_{2^{\prime}}\right), 2.72\left(\mathrm{~m}, 1 \mathrm{H}, \mathrm{H}_{2^{\prime}}\right), 4.52\left(\mathrm{~m}, 1 \mathrm{H}, \mathrm{H}_{4^{\prime}}\right), 4.69$ (dd, $1 \mathrm{H}, \mathrm{H}_{5^{\prime}}$, $\left.{ }^{3} \mathrm{~J}_{\mathrm{HH}} 4.0,{ }^{3} \mathrm{~J}_{\mathrm{HH}} 12.1 \mathrm{~Hz}\right), 4.74\left(\mathrm{dd}, 1 \mathrm{H}, \mathrm{H}_{5},{ }^{3} \mathrm{~J}_{\mathrm{HH}} 3.5,{ }^{3} \mathrm{~J}_{\mathrm{HH}} 12.1 \mathrm{~Hz}\right.$ ), 5.61 (apparent d, $\left.1 \mathrm{H}, \mathrm{H}_{3},{ }^{3} \mathrm{~J}_{\mathrm{HH}} 6.9 \mathrm{~Hz}\right), 6.42\left(\mathrm{dd}, 1 \mathrm{H}, \mathrm{H}_{1},{ }^{3} \mathrm{~J}_{\mathrm{HH}} 5.6,{ }^{3} \mathrm{~J}_{\mathrm{HH}} 8.8 \mathrm{~Hz}\right), 7.22\left(\mathrm{~s}, 1 \mathrm{H}, \mathrm{H}_{6}\right), 7.47$ $\left(\mathrm{m}, 4 \mathrm{H}, \mathrm{H}_{\mathrm{o}}\right), 7.99\left(\mathrm{~m}, 1 \mathrm{H}, \mathrm{H}_{\mathrm{m}}\right)$, and $8.54(\mathrm{~s}, 1 \mathrm{H}, \mathrm{NH}) ; \stackrel{13}{\mathrm{C}} \mathrm{NMR}\left(\mathrm{MeOH}-d_{4}, 100.6\right.$ $\mathrm{MHz}): \delta 12.3(\mathrm{Me}), 30.8\left(\mathrm{C}_{2^{\prime}}\right), 66.9\left(\mathrm{C}_{5^{\prime}}\right), 76.8\left(\mathrm{C}_{3^{\prime}}\right), 83.6,86.9\left(\mathrm{C}_{1^{\prime}}+\mathrm{C}_{4^{\prime}}\right), 112.0\left(\mathrm{C}_{5}\right)$, $129.8\left(\mathrm{C}_{\mathrm{p}}\right), 130.0,132.4\left(\mathrm{C}_{\mathrm{o}}+\mathrm{C}_{\mathrm{m}}\right), 137.3\left(\mathrm{C}_{6}\right), 141.0\left(\mathrm{C}_{\mathrm{i}}\right), 152.2\left(\mathrm{C}_{2}\right), 166.2\left(\mathrm{C}_{4}\right)$, and $166.6(\mathrm{C}=\mathrm{O}) ; \mathrm{MS}\left(\mathrm{ESI}^{+}, \mathrm{m} / \mathrm{z}\right): 520\left[(\mathrm{M}+\mathrm{H})^{+}, 10 \%\right]$ and $542\left[(\mathrm{M}+\mathrm{Na})^{+}, 20\right] ;$ Anal. Calcd. (\%) for $\mathrm{C}_{24} \mathrm{H}_{20} \mathrm{~N}_{2} \mathrm{O}_{7} \mathrm{Cl}_{2}$ : C, 55.51; $\mathrm{H}, 3.88 ; \mathrm{N}, 5.39$. Found: $\mathrm{C}, 55.2 ; \mathrm{H}, 4.0 ; \mathrm{N}$, 5.2 .

3'-O-(p-Chlorobenzoyl)- $\alpha$-D-thymidine (9). $\quad \underline{R}_{f}\left(10 \%{ }^{i} \mathrm{PrOH} / \mathrm{CH}_{2} \mathrm{Cl}_{2}\right): 0.25 ; \underline{\mathrm{IR}}$ $(\mathrm{NaCl}): \quad v \quad 3280,3100,1760$, and $1720 \mathrm{~cm}^{-1} ;{ }^{1} \mathrm{H}$ NMR $\left(\mathrm{MeOH}-d_{4}, 300 \mathrm{MHz}\right)$ : $\delta 1.99(\mathrm{~s}, 3 \mathrm{H}, \mathrm{Me}), 2.61\left(\mathrm{~m}, 1 \mathrm{H}, \mathrm{H}_{2}\right), 3.65\left(\mathrm{~m}, 1 \mathrm{H}, \mathrm{H}_{2^{\prime}}\right), 3.94$ (apparent $\mathrm{d}, 2 \mathrm{H}, \mathrm{H}_{5}$, ${ }^{3} \mathrm{~J}_{\mathrm{HH}} 3.5 \mathrm{~Hz}$ ), 4.90 (apparent $\mathrm{t}, 1 \mathrm{H}, \mathrm{H}_{4},{ }^{3} \mathrm{~J}_{\mathrm{HH}} 3.5 \mathrm{~Hz}$ ), 5.69 (apparent $\mathrm{d}, 1 \mathrm{H}, \mathrm{H}_{3},{ }^{3} \mathrm{~J}_{\mathrm{HH}}$ $5.9 \mathrm{~Hz}$ ), 6.42 (apparent d, $1 \mathrm{H}, \mathrm{H}_{1},{ }^{3} \mathrm{~J}_{\mathrm{HH}} 5.5 \mathrm{~Hz}$ ), 7.65 (apparent d, $2 \mathrm{H}, \mathrm{H}_{\mathrm{o}},{ }^{3} \mathrm{~J}_{\mathrm{HH}} 8.6$ $\mathrm{Hz}), 7.82\left(\mathrm{~s}, 1 \mathrm{H}, \mathrm{H}_{6}\right)$, and 8.07 (apparent $\mathrm{d}, 2 \mathrm{H}, \mathrm{H}_{\mathrm{m}},{ }^{3} \mathrm{~J}_{\mathrm{HH}} 8.6 \mathrm{~Hz}$ ); ${ }^{13} \underline{\mathrm{C}} \mathrm{NMR}$ $\left(\mathrm{MeOH}-d_{4}, 100.6 \mathrm{MHz}\right): \delta 12.6(\mathrm{Me}), 40.0\left(\mathrm{C}_{2^{\prime}}\right), 63.4\left(\mathrm{C}_{5^{\prime}}\right), 77.2\left(\mathrm{C}_{3^{\prime}}\right), 89.2,89.5$ $\left(\mathrm{C}_{1},+\mathrm{C}_{4^{\prime}}\right), 110.5\left(\mathrm{C}_{5}\right), 129.5\left(\mathrm{C}_{\mathrm{p}}\right), 130.0,132.1\left(\mathrm{C}_{\mathrm{o}}+\mathrm{C}_{\mathrm{m}}\right), 137.9\left(\mathrm{C}_{6}\right), 140.9\left(\mathrm{C}_{\mathrm{i}}\right), 152.1$ $\left(\mathrm{C}_{2}\right), 166.1\left(\mathrm{C}_{4}\right)$, and $166.6(\mathrm{C}=\mathrm{O}) ; \mathrm{MS}\left(\mathrm{ESI}^{+}, \mathrm{m} / \mathrm{z}\right): 403\left[(\mathrm{M}+\mathrm{Na})^{+}, 100 \%\right]$; Anal. Calcd. (\%) for $\mathrm{C}_{17} \mathrm{H}_{17} \mathrm{~N}_{2} \mathrm{O}_{6} \mathrm{Cl}: \mathrm{C}, 53.62 ; \mathrm{H}, 4.50 ; \mathrm{N}, 7.36$. Found: $\mathrm{C}, 53.9 ; \mathrm{H}, 4.6 ; \mathrm{N}$, 7.2 .

General procedure for the enzymatic hydrolysis of 1-(3',5'-di-O-benzoyl2'-fluoro-2'-deoxy- $\alpha / \beta-D$-arabinofuranosyl)thymine (10/11). To a solution of $10 / 11(0.214 \mathrm{mmol})$ in an appropriate solvent was added phosphate buffer $\mathrm{pH} 7$ and the corresponding enzyme. Reaction concentration: $0.1 \mathrm{M}$. The temperature, reaction time, and ratios of enzyme, buffer, and co-solvent are indicated in Table 3). The reaction mixture was stirred at $250 \mathrm{rpm}$, and monitored by HPLC (Method C). The enzyme was filtered off, washed with $\mathrm{CH}_{2} \mathrm{Cl}_{2}$ and $\mathrm{MeOH}$, and the solvents evaporated under vacuum. The residue was taken up in $\mathrm{NaHCO}_{3}$ (aq) and extracted with $\mathrm{CH}_{2} \mathrm{Cl}_{2}$. The combined organic layers were dried over $\mathrm{Na}_{2} \mathrm{SO}_{4}$ and evaporated. The crude was subjected to flash cromatography $\left(3 \%{ }^{i} \mathrm{PrOH} / \mathrm{CHCl}_{3}\right)$ to give 12 in $82 \%$ yield and 13 in $86 \%$ yield (entry 10, Table 3 ). 
1-(3',5'-Di-O-benzoyl-2'-fluoro-2'-deoxy- $\alpha / \beta$-D-arabinofuranosyl)thymine 10( $\alpha): 11(\beta) . \underline{R}_{f}\left(4 \%{ }^{i} \mathrm{PrOH} / \mathrm{CHCl}_{3}\right): 0.35 ;{ }^{1} \underline{\mathrm{H} \mathrm{NMR}}\left(\mathrm{CDCl}_{3}, 400 \mathrm{MHz}\right): \delta 1.73(\mathrm{~s}, 3 \mathrm{H}$, $\left.\mathrm{Me}_{\beta}\right), 1.86\left(\mathrm{~s}, 3 \mathrm{H}, \mathrm{Me}_{\alpha}\right), 4.50\left(\mathrm{~m}, 1 \mathrm{H}, \mathrm{H}_{4^{\prime} \beta}\right), 4.62\left(\mathrm{~m}, 2 \mathrm{H}, \mathrm{H}_{5^{\prime} \alpha}\right), 4.83\left(\mathrm{~m}, 2 \mathrm{H}, \mathrm{H}_{5^{\prime} \beta}\right)$, $5.00\left(\mathrm{~m}, 1 \mathrm{H}, \mathrm{H}_{4^{\prime} \alpha}\right), 5.35$ (dd, $\left.1 \mathrm{H}, \mathrm{H}_{2}{ }^{\beta},{ }^{2} \mathrm{~J}_{\mathrm{HF}} 50.4 \mathrm{~Hz},{ }^{3} \mathrm{~J}_{\mathrm{HH}} 2.8 \mathrm{~Hz}\right), 5.54$ (apparent d, $1 \mathrm{H}, \mathrm{H}_{2^{\prime} \alpha},{ }^{2} \mathrm{~J}_{\mathrm{HF}} 49.2 \mathrm{~Hz}$ ), 5.64 (dd, $1 \mathrm{H}, \mathrm{H}_{3^{\prime} \beta}{ }^{3} \mathrm{~J}_{\mathrm{HF}} 18.0 \mathrm{~Hz},{ }^{3} \mathrm{~J}_{\mathrm{HH}} 2.8 \mathrm{~Hz}$ ), 5.71 (apparent $\mathrm{d}, 1 \mathrm{H}, \mathrm{H}_{3^{\prime} \alpha},{ }^{3} \mathrm{~J}_{\mathrm{HF}} 16.4 \mathrm{~Hz}$ ), 6.18 (apparent d, $1 \mathrm{H}, \mathrm{H}_{1^{\prime} \alpha},{ }^{3} \mathrm{~J}_{\mathrm{HF}} 15.2 \mathrm{~Hz}$ ), 6.37 (dd, $1 \mathrm{H}$, $\left.\mathrm{H}_{1^{\prime} \beta},{ }^{3} J_{\mathrm{HF}} 22.0 \mathrm{~Hz},{ }^{3} \mathrm{~J}_{\mathrm{HH}} 2.8 \mathrm{~Hz}\right), 7.25\left(\mathrm{~s}, 1 \mathrm{H}, \mathrm{H}_{6 \alpha}\right), 7.39\left(\mathrm{~s}, 1 \mathrm{H}, \mathrm{H}_{6 \beta}\right), 7.42-7.63(\mathrm{~m}$, $\left.12 \mathrm{H}, \mathrm{H}_{\mathrm{m}}+\mathrm{H}_{\mathrm{p}}\right), 7.88\left(\mathrm{~m}, 2 \mathrm{H}, \mathrm{H}_{\circ}\right), 7.95-8.15\left(\mathrm{~m}, 6 \mathrm{H}, \mathrm{H}_{\mathrm{o}}\right), 9.97$ (br s, $\left.1 \mathrm{H}, \mathrm{NH}_{\beta}\right)$, and $10.11\left(\mathrm{br} \mathrm{s}, 1 \mathrm{H}, \mathrm{NH}_{\alpha}\right) ; \stackrel{13}{\mathrm{C} \mathrm{NMR}}\left(\mathrm{CDCl}_{3}, 100.6 \mathrm{MHz}\right): \delta 12.3\left(\mathrm{Me}_{\beta}\right), 12.5\left(\mathrm{Me}_{\alpha}\right)$, $63.2\left(\mathrm{C}_{5^{\prime} \beta}\right), 63.5\left(\mathrm{C}_{5^{\prime} \alpha}\right), 76.4\left(\mathrm{~d}, \mathrm{C}_{3^{\prime}}{ }^{\alpha},{ }^{2} \mathrm{~J}_{\mathrm{CF}} 30.2 \mathrm{~Hz}\right), 76.6\left(\mathrm{~d}, \mathrm{C}_{3^{\prime} \beta},{ }^{2} \mathrm{~J}_{\mathrm{CF}} 20.1 \mathrm{~Hz}\right), 81.2$ $\left(\mathrm{C}_{4^{\prime} \beta}\right), 84.5\left(\mathrm{~d}, \mathrm{C}_{1^{\prime} \beta},{ }^{2} \mathrm{~J}_{\mathrm{CF}} 17.1 \mathrm{~Hz}\right), 84.8\left(\mathrm{C}_{4^{\prime} \alpha}\right), 91.8\left(\mathrm{~d}, \mathrm{C}_{1^{\prime} \alpha},{ }^{2} \mathrm{~J}_{\mathrm{CF}} 37.2 \mathrm{~Hz}\right), 92.8(\mathrm{~d}$, $\left.\mathrm{C}_{2^{\prime} \beta},{ }^{1} \mathrm{~J}_{\mathrm{CF}} 191.1 \mathrm{~Hz}\right), 97.4\left(\mathrm{~d}, \mathrm{C}_{2^{\prime} \alpha},{ }^{1} \mathrm{~J}_{\mathrm{CF}} 181.1 \mathrm{~Hz}\right), 110.6\left(\mathrm{C}_{5 \beta}\right), 110.9\left(\mathrm{C}_{5 \alpha}\right), 128.2$, 128.5, 128.6, 128.7, 129.4, 129.7, 129.8, $130.0\left(\mathrm{C}_{\mathrm{i}}+\mathrm{C}_{\mathrm{m}}+\mathrm{C}_{\mathrm{o}}\right), 133.4,133.7,134.1$ $\left(\mathrm{C}_{\mathrm{p}}\right), 135.7\left(\mathrm{C}_{6 \alpha}\right), 136.4\left(\mathrm{~d}, \mathrm{C}_{6 \beta},{ }^{3} \mathrm{~J}_{\mathrm{CF}} 3.7 \mathrm{~Hz}\right), 151.5\left(\mathrm{C}_{2 \alpha}\right), 151.6\left(\mathrm{C}_{2 \beta}\right), 164.0\left(\mathrm{C}_{4 \beta}\right)$, $164.3\left(\mathrm{C}_{4 \alpha}\right), 164.9,165.3,166.2(\mathrm{C}=\mathrm{O}) ; \stackrel{19}{\mathrm{~F} N M R}\left(\mathrm{CDCl}_{3}, 282.4 \mathrm{MHz}\right) \delta-200.74$ $(\alpha),-186.21(\beta)$.

1-(3'-O-Benzoyl-2'-fluoro-2'-deoxy- $\beta$-D-arabinofuranosyl)thymine (12). $\underline{R}_{f}$ (4\% $\left.\mathrm{MeOH} / \mathrm{CHCl}_{3}\right): 0.20 ; \underline{\mathrm{mp}}: 192-193^{\circ} \mathrm{C}$; IR $(\mathrm{KBr}): \mathrm{v} 3420,3350,3166,3029,2837$, 1710, 1658, 1294, 1268, 1123 and $716 \mathrm{~cm}^{-1}$; ${ }^{1} \mathrm{H} \mathrm{NMR}\left(\mathrm{CDCl}_{3}, 400 \mathrm{MHz}\right): \delta 1.97$ (d, $\left.3 \mathrm{H}, \mathrm{Me},\left|{ }^{4} \mathrm{~J}_{\mathrm{HH}}\right| 0.9 \mathrm{~Hz}\right), 2.25(\mathrm{br} \mathrm{s}, 1 \mathrm{H}, \mathrm{OH}), 4.05\left(\mathrm{~m}, 2 \mathrm{H}, \mathrm{H}_{5^{\prime}}\right), 4.20\left(\mathrm{~m}, 1 \mathrm{H}, \mathrm{H}_{4^{\prime}}\right)$, 5.34 (ddd, $1 \mathrm{H}, \mathrm{H}_{2},{ }^{2} J_{\mathrm{HF}} 50.8 \mathrm{~Hz},{ }^{3} \mathrm{~J}_{\mathrm{HH}} 3.2,1.7 \mathrm{~Hz}$ ), 5.54 (ddd, $1 \mathrm{H}, \mathrm{H}_{3},{ }^{3} \mathrm{~J}_{\mathrm{HF}} 19.6 \mathrm{~Hz}$, $\left.{ }^{3} J_{\mathrm{HH}} 4.4,1.7 \mathrm{~Hz}\right), 6.32\left(\mathrm{dd}, 1 \mathrm{H}, \mathrm{H}_{1},{ }^{3} J_{\mathrm{HF}} 19.8 \mathrm{~Hz},{ }^{3} \mathrm{~J}_{\mathrm{HH}} 3.2 \mathrm{~Hz}\right), 7.45\left(\mathrm{~s}, 1 \mathrm{H}, \mathrm{H}_{6}\right), 7.51$ $\left(\mathrm{t}, 2 \mathrm{H}, \mathrm{H}_{\mathrm{m}},{ }^{3} \mathrm{~J}_{\mathrm{HH}} 7.3 \mathrm{~Hz}\right), 7.65\left(\mathrm{tt}, 1 \mathrm{H}, \mathrm{H}_{\mathrm{p}},{ }^{3} \mathrm{~J}_{\mathrm{HH}} 7.3 \mathrm{~Hz},\left.\right|^{4} \mathrm{~J}_{\mathrm{HH}} \mid 1.2 \mathrm{~Hz}\right), 8.06(\mathrm{dd}, 2 \mathrm{H}$,

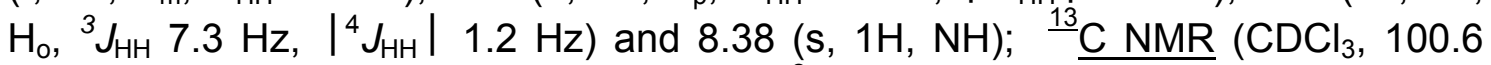
$\mathrm{MHz}): \delta 12.6(\mathrm{Me}), 62.0\left(\mathrm{C}_{5^{\prime}}\right), 76.2\left(\mathrm{~d}, \mathrm{C}_{3^{\prime}},{ }^{2} \mathrm{~J}_{\mathrm{CF}} 29.8 \mathrm{~Hz}\right), 82.6\left(\mathrm{C}_{4^{\prime}}\right), 83.7\left(\mathrm{~d}, \mathrm{C}_{1^{\prime}}\right.$, $\left.{ }^{2} J_{\mathrm{CF}} 16.8 \mathrm{~Hz}\right), 93.1\left(\mathrm{~d}, \mathrm{C}_{2},{ }^{1} \mathrm{~J}_{\mathrm{CF}} 193.2 \mathrm{~Hz}\right), 110.8\left(\mathrm{C}_{5}\right), 128.3\left(\mathrm{C}_{\mathrm{i}}\right), 128.8\left(\mathrm{C}_{\mathrm{m}}\right), 130.0$ $\left(\mathrm{C}_{\mathrm{o}}\right), 134.2\left(\mathrm{C}_{\mathrm{p}}\right), 136.4\left(\mathrm{~d}, \mathrm{C}_{6},{ }^{3} \mathrm{~J}_{\mathrm{CF}} 3.9 \mathrm{~Hz}\right), 150.2\left(\mathrm{C}_{2}\right), 163.4\left(\mathrm{C}_{4}\right), 165.7(\mathrm{C}=\mathrm{O}) ;{ }^{19} \mathrm{~F}$ NMR $\left(\mathrm{CDCl}_{3}, 282.4 \mathrm{MHz}\right) \delta-200.26 ; \underline{\mathrm{MS}}\left(\mathrm{ESI}^{+}, \mathrm{m} / \mathrm{z}\right): 365\left[(\mathrm{M}+\mathrm{H})^{+}, 80 \%\right]$; Anal. Calcd. (\%) for $\mathrm{C}_{17} \mathrm{H}_{17} \mathrm{FN}_{2} \mathrm{O}_{6}$ : C, 56.04; $\mathrm{H}, 4.7 ; \mathrm{N}, 7.69 ; \mathrm{O}, 26.35 ; \mathrm{F}, 5.21$. Found: $\mathrm{C}$, $56.1 ; \mathrm{H}, 4.7 ; \mathrm{N}, 7.5 ; \mathrm{O}, 26.2 ; \mathrm{F}, 5.2$.

1-(3'-O-Benzoyl-2'-fluoro-2'-deoxy- $\alpha$-D-arabinofuranosyl)thymine (13). $R_{f}(4 \%$ $\left.\mathrm{MeOH} / \mathrm{CHCl}_{3}\right): 0.13$; $\underline{\mathrm{mp}}: 188-189^{\circ} \mathrm{C}$; IR $(\mathrm{KBr}): \mathrm{v} 3469,3147,3033,2896,2812$, $1725,1688,1274,1121$, and $710 \mathrm{~cm}^{-1} ;{ }^{1} \underline{\mathrm{H} \mathrm{NMR}}\left(\mathrm{MeOH}-d_{4}, 400 \mathrm{MHz}\right): \delta 2.05$ (d, $\left.3 \mathrm{H}, \mathrm{Me},\left|{ }^{4} \mathrm{~J}_{\mathrm{HH}}\right| 1.2 \mathrm{~Hz}\right), 4.00\left(\mathrm{~m}, 2 \mathrm{H}, \mathrm{H}_{5^{\prime}}\right), 4.95\left(\mathrm{~m}, 1 \mathrm{H}, \mathrm{H}_{4}{ }^{\prime}\right), 5.69\left(\mathrm{~d}, 1 \mathrm{H}, \mathrm{H}_{2},{ }^{2} \mathrm{~J}_{\mathrm{HF}}\right.$ $49.1 \mathrm{~Hz}), 5.80\left(\mathrm{dt}, 1 \mathrm{H}, \mathrm{H}_{3},{ }^{3} J_{\mathrm{HF}} 16.1 \mathrm{~Hz},{ }^{3} \mathrm{~J}_{\mathrm{HH}} 1.7 \mathrm{~Hz}\right), 6.30\left(\mathrm{dd}, 1 \mathrm{H}, \mathrm{H}_{1},{ }^{3} \mathrm{~J}_{\mathrm{HF}} 14.9\right.$ $\left.\mathrm{Hz},{ }^{3} J_{\mathrm{HH}} 1.2 \mathrm{~Hz}\right), 7.67\left(\mathrm{t}, 2 \mathrm{H}, \mathrm{H}_{\mathrm{m}},{ }^{3} \mathrm{~J}_{\mathrm{HH}} 8.0 \mathrm{~Hz}\right), 7.76\left(\mathrm{~d}, 1 \mathrm{H}, \mathrm{H}_{6},\left.\right|^{4} J_{\mathrm{HH}} \mid 1.2 \mathrm{~Hz}\right), 7.83$ (tt, $\left.1 \mathrm{H}, \mathrm{H}_{\mathrm{p}},{ }^{3} \mathrm{~J}_{\mathrm{HH}} 8.0 \mathrm{~Hz},\left|{ }^{4} \mathrm{~J}_{\mathrm{HH}}\right| 1.2 \mathrm{~Hz}\right)$ and $8.15\left(\mathrm{dd}, 2 \mathrm{H}, \mathrm{H}_{\mathrm{o}},{ }^{3} \mathrm{~J}_{\mathrm{HH}} 8.0 \mathrm{~Hz},\left|{ }^{4} J_{\mathrm{HH}}\right|\right.$ $1.2 \mathrm{~Hz}) ; \stackrel{13}{\mathrm{C} ~ N M R}\left(\mathrm{MeOH}-\mathrm{d}_{4}, 100.6 \mathrm{MHz}\right): \delta 12.6(\mathrm{Me}), 62.8\left(\mathrm{C}_{5}\right), 77.5\left(\mathrm{~d}, \mathrm{C}_{3^{\prime}},{ }^{2} \mathrm{~J}_{\mathrm{CF}}\right.$ $29.3 \mathrm{~Hz}$ ), $88.7\left(\mathrm{C}_{4^{\prime}}\right), 92.4\left(\mathrm{~d}, \mathrm{C}_{1^{\prime}},{ }^{2} \mathrm{~J}_{\mathrm{CF}} 36.6 \mathrm{~Hz}\right), 98.9\left(\mathrm{~d}, \mathrm{C}_{2^{\prime}},{ }^{1} \mathrm{~J}_{\mathrm{CF}} 186.0 \mathrm{~Hz}\right), 111.4$ $\left(\mathrm{C}_{5}\right), 129.9\left(\mathrm{C}_{\mathrm{m}}\right), 130.3\left(\mathrm{C}_{\mathrm{i}}\right), 130.9\left(\mathrm{C}_{\mathrm{o}}\right), 135.1\left(\mathrm{C}_{\mathrm{p}}\right), 138.0\left(\mathrm{C}_{6}\right), 152.5\left(\mathrm{C}_{2}\right), 166.5$, $166.6\left(\mathrm{C}_{4}+\mathrm{C}=\mathrm{O}\right) ; \stackrel{19}{\mathrm{~F} \mathrm{NMR}}\left(\mathrm{MeOH}-\mathrm{d}_{4}, 282.4 \mathrm{MHz}\right) \delta-189.61 \underline{\mathrm{MS}}\left(\mathrm{ESI}^{+}, \mathrm{m} / \mathrm{z}\right): 365$ $\left[(\mathrm{M}+\mathrm{H})^{+}, 90 \%\right]$; Anal. Calcd. (\%) for $\mathrm{C}_{17} \mathrm{H}_{17} \mathrm{FN}_{2} \mathrm{O}_{6}: \mathrm{C}, 56.04 ; \mathrm{H}, 4.7 ; \mathrm{N}, 7.69 ; \mathrm{O}$, 26.35; F, 5.21. Found: C, 56.2; $\mathrm{H}, 4.7 ; \mathrm{N}, 7.6$; O, 26.3; F, 5.3.

1-(2'-Fluoro-2'-deoxy- $\alpha / \beta-D-a r a b i n o f u r a n o s y l) t h y m i n e \quad(14 / 15) . \quad$ Sodium methoxide ( $252 \mathrm{mg}, 4.487 \mathrm{mmol}$ ) was added to a solution of 10/11 (350 mg, 0.048 $\mathrm{mmol})$ in anhydrous $\mathrm{MeOH}(4.9 \mathrm{~mL})$. The mixture was stirred at $\mathrm{rt}$ during $1 \mathrm{~h}$. The 
reaction was neutralized with Dowex $50 \mathrm{~W} x 4-400$ ion-exchange resin. Then, the residue was filtered off, washed with $\mathrm{MeOH}$, and the solvent evaporated under vacuum. The crude product was subjected to flash chromatography (50-100\% EtOAc/hexane) to afford 14/15 (mixture $\alpha / \beta$ 40:60) in 93\% yield. $\underline{R}_{f}$ (EtOAc): 0.19 ; ${ }^{1} \underline{\mathrm{H} \mathrm{NMR}}\left(\mathrm{MeOH}-d_{4}, 400 \mathrm{MHz}\right): \delta 2.06,2.07(2 \mathrm{~s}, 6 \mathrm{H}, 2 \mathrm{Me}), 3.87\left(\mathrm{~m}, 2 \mathrm{H}, \mathrm{H}_{5}{ }^{\prime}\right), 4.03$ $\left(\mathrm{m}, 2 \mathrm{H}, \mathrm{H}_{5^{\prime} \alpha}\right), 4.10\left(\mathrm{~m}, 1 \mathrm{H}, \mathrm{H}_{4^{\prime} \beta}\right), 4.47-4.56\left(\mathrm{~m}, 3 \mathrm{H}, \mathrm{H}_{3^{\prime} \beta}+\mathrm{H}_{3^{\prime} \alpha}+\mathrm{H}_{4^{\prime} \alpha}\right), 5.21$ (ddd, 1H, $\left.\mathrm{H}_{2^{\prime} \beta},{ }^{2} J_{\mathrm{HF}} 52.3 \mathrm{~Hz},{ }^{3} J_{\mathrm{HH}} 4.0,2.6 \mathrm{~Hz}\right), 5.39$ (dt, $1 \mathrm{H}, \mathrm{H}_{2^{\prime} \alpha},{ }^{2} J_{\mathrm{HF}} 51.3 \mathrm{~Hz},{ }^{3} J_{\mathrm{HH}} 2.5 \mathrm{~Hz}$ ), $6.19\left(\mathrm{dd}, 1 \mathrm{H}, \mathrm{H}_{1}{ }^{\prime},{ }^{3} \mathrm{~J}_{\mathrm{HF}} 16.3 \mathrm{~Hz},{ }^{3} \mathrm{~J}_{\mathrm{HH}} 2.5 \mathrm{~Hz}\right), 6.36\left(\mathrm{dd}, 1 \mathrm{H}, \mathrm{H}_{1}{ }^{\prime} \beta,{ }^{3} J_{\mathrm{HF}} 17.0 \mathrm{~Hz},{ }^{3} J_{\mathrm{HH}}\right.$ $4.0 \mathrm{~Hz}$ ), $7.72(\mathrm{~s}, 1 \mathrm{H}, \mathrm{NH}), 7.86(\mathrm{~s}, 1 \mathrm{H}, \mathrm{NH}) ; \stackrel{13}{\mathrm{C}} \mathrm{NMR}\left(\mathrm{MeOH}-d_{4}, 100.6 \mathrm{MHz}\right): \delta$ $12.4\left(\mathrm{Me}_{\alpha}\right), 12.5\left(\mathrm{Me}_{\beta}\right), 61.8\left(\mathrm{C}_{5^{\prime} \beta}\right), 62.5\left(\mathrm{C}_{5^{\prime} \alpha}\right), 74.8\left(\mathrm{~d}, \mathrm{C}_{3^{\prime} \beta},{ }^{2} \mathrm{~J}_{\mathrm{CF}} 25.0 \mathrm{~Hz}\right), 75.1$ (d, $\mathrm{C}_{3^{\prime} \beta},{ }^{2} J_{\mathrm{CF}} 24.1 \mathrm{~Hz}$ ), $85.0\left(\mathrm{~d}, \mathrm{C}_{1{ }^{\prime} \beta},{ }^{2} \mathrm{~J}_{\mathrm{CF}} 17.1 \mathrm{~Hz}\right), 85.3\left(\mathrm{~d}, \mathrm{C}_{4}{ }^{\prime} \beta,{ }^{3} J_{\mathrm{CF}} 4.0 \mathrm{~Hz}\right), 89.5$ (d, $\left.\mathrm{C}_{4^{\prime} \alpha},{ }^{3} J_{\mathrm{CF}} 2.0 \mathrm{~Hz}\right), 91.6\left(\mathrm{~d}, \mathrm{C}_{1^{\prime} \alpha},{ }^{2} \mathrm{~J}_{\mathrm{CF}} 37.2 \mathrm{~Hz}\right), 97.0\left(\mathrm{~d}, \mathrm{C}_{2^{\prime} \beta},{ }^{1} \mathrm{~J}_{\mathrm{CF}} 191.1 \mathrm{~Hz}\right), 101.0(\mathrm{~d}$, $\left.\mathrm{C}_{2^{\prime} \alpha},{ }^{1} J_{\mathrm{CF}} 186.1 \mathrm{~Hz}\right), 110.7\left(\mathrm{C}_{5 \beta}\right), 111.2\left(\mathrm{C}_{5 \alpha}\right), 138.7\left(\mathrm{C}_{6 \beta}\right), 138.8\left(\mathrm{C}_{6 \alpha}\right), 152.1\left(\mathrm{C}_{2 \beta}\right)$, $152.3\left(\mathrm{C}_{2 \alpha}\right), 166.3\left(\mathrm{C}_{4 \beta}\right)$ and $166.4\left(\mathrm{C}_{4 \alpha}\right)$; ${ }^{19} \mathrm{~F} \mathrm{NMR}\left(\mathrm{MeOH}-d_{4}, 282.4 \mathrm{MHz}\right) \delta-$ $200.72(\alpha),-189.55(\beta)$.

Enzymatic acylation of 1-(2'-fluoro-2'-deoxy- $\alpha / \beta-D$ arabinofuranosyl)thymine (14/15). In a standard procedure, solvent $(1 \mathrm{~mL})$ was added to an Erlenmeyer flask that contained 14/15 $(0.1 \mathrm{mmol})$, O-levulinyl acetonoxime or vinyl benzoate (3 equiv), and the enzyme under nitrogen (ratio substrate:CAL-B is $1: 1 \mathrm{w} / \mathrm{w}$; ratio substrate:PSL-C is $1: 3 \mathrm{w} / \mathrm{w})$. The reaction mixture was stirred at $250 \mathrm{rpm}$ and $60{ }^{\circ} \mathrm{C}$, and monitored by HPLC (Method C). The enzyme was filtered off, washed with $\mathrm{CH}_{2} \mathrm{Cl}_{2}$ and $\mathrm{MeOH}$, and the solvents evaporated under vacuum to afford an $\alpha / \beta$ mixture of 5 '-O-acyl derivatives. A successful separation by flash chromatography was not achieved.

1-(5'-O-Benzoyl-2'-fluoro-2'-deoxy- $\alpha$-D-arabinofuranosyl)thymine (18). $\underline{R}_{f}(4 \%$ $\left.{ }^{i} \mathrm{PrOH} / \mathrm{CHCl}_{3}\right): 0.10$; IR (KBr): v 3406, 2962, 2934, 1712, 1693, 1661, 1274, 1094 and $708 \mathrm{~cm}^{-1} ;{ }^{1}{ }^{\mathrm{H}} \mathrm{NMR}\left(\mathrm{MeOH}-d_{4}, 600 \mathrm{MHz}\right): \delta 1.90\left(\mathrm{~d}, 3 \mathrm{H}, \mathrm{Me},\left|{ }^{4} \mathrm{~J}_{\mathrm{HH}}\right| 1.2 \mathrm{~Hz}\right)$, $4.46\left(\mathrm{c}, 1 \mathrm{H}, \mathrm{H}_{4},{ }^{3} \mathrm{~J}_{\mathrm{HH}} 3.6 \mathrm{~Hz}\right), 4.66$ (ddd, $1 \mathrm{H}, \mathrm{H}_{3},{ }^{3} \mathrm{~J}_{\mathrm{HF}} 17.8 \mathrm{~Hz},{ }^{3} \mathrm{~J}_{\mathrm{HH}} 3.6,1.7 \mathrm{~Hz}$ ), $4.84\left(\mathrm{~m}, 2 \mathrm{H}, \mathrm{H}_{5}\right), 5.23$ (ddd, $1 \mathrm{H}, \mathrm{H}_{2}$, $\left.{ }^{2} J_{\mathrm{HF}} 52.0 \mathrm{~Hz},{ }^{3} J_{\mathrm{HH}} 3.4,1.7 \mathrm{~Hz}\right), 6.42(\mathrm{dd}, 1 \mathrm{H}$, $\left.\mathrm{H}_{1},{ }^{3}{ }^{3} \mathrm{HF}_{2} 20.0 \mathrm{~Hz},{ }^{3} \mathrm{~J}_{\mathrm{HH}} 3.4 \mathrm{~Hz}\right), 7.67\left(\mathrm{~m}, 1 \mathrm{H}, \mathrm{H}_{6}\right), 7.76\left(\mathrm{t}, 2 \mathrm{H}, \mathrm{H}_{\mathrm{m}},{ }^{3} \mathrm{~J}_{\mathrm{HH}} 8.3 \mathrm{~Hz}\right), 7.83$ (tt, $\left.1 \mathrm{H}, \mathrm{H}_{\mathrm{p}},{ }^{3} \mathrm{~J}_{\mathrm{HH}} 8.3 \mathrm{~Hz},\left|{ }^{4} \mathrm{~J}_{\mathrm{HH}}\right| 1.3 \mathrm{~Hz}\right)$ and $8.27\left(\mathrm{dd}, 2 \mathrm{H}, \mathrm{H}_{\mathrm{o}},{ }^{3} \mathrm{~J}_{\mathrm{HH}} 8.3 \mathrm{~Hz},\left|{ }^{4} J_{\mathrm{HH}}\right|\right.$ $1.3 \mathrm{~Hz}) ;{ }^{13} \mathrm{C} \mathrm{NMR}\left(\mathrm{MeOH}-d_{4}, 150.9 \mathrm{MHz}\right): \delta 12.5(\mathrm{Me}), 64.6\left(\mathrm{C}_{5^{\prime}}\right), 76.0\left(\mathrm{~d}, \mathrm{C}_{3^{\prime}},{ }^{2} \mathrm{~J}_{\mathrm{CF}}\right.$ $25.7 \mathrm{~Hz}$ ), $84.1\left(\mathrm{C}_{4^{\prime}}\right), 85.9\left(\mathrm{~d}, \mathrm{C}_{1},{ }^{2} \mathrm{~J}_{\mathrm{CF}} 16.6 \mathrm{~Hz}\right), 96.6\left(\mathrm{~d}, \mathrm{C}_{2},{ }^{1} \mathrm{~J}_{\mathrm{CF}} 190.1 \mathrm{~Hz}\right), 110.8$ $\left(\mathrm{C}_{5}\right), 129.9\left(\mathrm{C}_{\mathrm{m}}\right), 131.0\left(\mathrm{C}_{\mathrm{o}}\right), 131.2\left(\mathrm{C}_{\mathrm{i}}\right), 134.7\left(\mathrm{C}_{\mathrm{p}}\right), 138.0\left(\mathrm{~d}, \mathrm{C}_{6},{ }^{4} \mathrm{~J}_{\mathrm{CF}} 3.0 \mathrm{~Hz}\right), 152.2$ $\left(\mathrm{C}_{2}\right), 166.4\left(\mathrm{C}_{4}\right)$ and $167.8(\mathrm{C}=\mathrm{O}) ; \stackrel{19}{\mathrm{~F} N M R}\left(\mathrm{MeOH}-d_{4}, 282.4 \mathrm{MHz}\right) \delta-202.01 ; \underline{\mathrm{MS}}$ $\left(\mathrm{ESI}^{+}, \mathrm{m} / \mathrm{z}\right): 365\left[(\mathrm{M}+\mathrm{H})^{+}, 90 \%\right]$; Anal. Calcd. (\%) for $\mathrm{C}_{17} \mathrm{H}_{17} \mathrm{FN}_{2} \mathrm{O}_{6}: \mathrm{C}, 56.04 ; \mathrm{H}$, 4.7; N, 7.69; O, 26.35; F, 5.21. Found: C, 56.1; H, 4.6; N, 7.6; O, 26.4; F, 5.2.

1-(5'-O-Benzoyl-2'-fluoro-2'-deoxy- $\beta$-D-arabinofuranosyl)thymine (19). $\underline{R}_{f}(4 \%$ $\left.{ }^{i} \mathrm{PrOH} / \mathrm{CHCl}_{3}\right): 0.12$; $\mathrm{mp}: 73-74^{\circ} \mathrm{C}$; IR $(\mathrm{KBr}):$ v 3415, 3208, 3247, 3057, 2925, 1717, 1693, 1273, 1097 and $713 \mathrm{~cm}^{-1}$; $\underline{\mathrm{H} ~ \mathrm{NMR}}\left(\mathrm{MeOH}-d_{4}, 400 \mathrm{MHz}\right): \delta 2.06(\mathrm{~d}$, $\left.3 \mathrm{H}, \mathrm{Me},\left|{ }^{4} \mathrm{~J}_{\mathrm{HH}}\right| 1.1 \mathrm{~Hz}\right), 4.64\left(\mathrm{~m}, 1 \mathrm{H}, \mathrm{H}_{3^{\prime}}\right), 4.68\left(\mathrm{~m}, 2 \mathrm{H}, \mathrm{H}_{5^{\prime}}\right), 4.91\left(\mathrm{~m}, 1 \mathrm{H}, \mathrm{H}_{4^{\prime}}\right), 5.45$ (dt, $\left.1 \mathrm{H}, \mathrm{H}_{2},{ }^{2} \mathrm{~J}_{\mathrm{HF}} 51.0 \mathrm{~Hz},{ }^{3} \mathrm{~J}_{\mathrm{HH}} 2.1 \mathrm{~Hz}\right), 6.21\left(\mathrm{dd}, 1 \mathrm{H}, \mathrm{H}_{1},{ }^{3} \mathrm{~J}_{\mathrm{HF}} 16.8 \mathrm{~Hz},{ }^{3} \mathrm{~J}_{\mathrm{HH}} 2.1 \mathrm{~Hz}\right.$ ), $7.66\left(\mathrm{t}, 2 \mathrm{H}, \mathrm{H}_{\mathrm{m}},{ }^{3} \mathrm{~J}_{\mathrm{HH}} 7.9 \mathrm{~Hz}\right), 7.75\left(\mathrm{~d}, 1 \mathrm{H}, \mathrm{H}_{6},\left|{ }^{4} \mathrm{~J}_{\mathrm{HH}}\right| 1.1 \mathrm{~Hz}\right), 7.80\left(\mathrm{tt}, 1 \mathrm{H}, \mathrm{H}_{\mathrm{p}},{ }^{3} \mathrm{~J}_{\mathrm{HH}}\right.$ $\left.7.9 \mathrm{~Hz},\left|{ }^{4} \mathrm{~J}_{\mathrm{HH}}\right| 1.3 \mathrm{~Hz}\right)$, and $8.24\left(\mathrm{dd}, 2 \mathrm{H}, \mathrm{H}_{\mathrm{O}},{ }^{3} \mathrm{~J}_{\mathrm{HH}} 7.9 \mathrm{~Hz},\left|{ }^{4} \mathrm{~J}_{\mathrm{HH}}\right| 1.3 \mathrm{~Hz}\right) ;{ }^{13} \underline{\mathrm{C}}$ NMR $\left(\mathrm{MeOH}-d_{4}, 100.6 \mathrm{MHz}\right): \delta 12.6(\mathrm{Me}), 65.1\left(\mathrm{C}_{5}\right), 75.9\left(\mathrm{~d}, \mathrm{C}_{3^{3}},{ }^{2} \mathrm{~J}_{\mathrm{CF}} 25.2 \mathrm{~Hz}\right)$, $\overline{87.1}\left(\mathrm{~d}, \mathrm{C}_{4},{ }^{3} \mathrm{~J}_{\mathrm{CF}} 3.0 \mathrm{~Hz}\right), 92.2\left(\mathrm{~d}, \mathrm{C}_{1},{ }^{2} \mathrm{~J}_{\mathrm{CF}} 36.2 \mathrm{~Hz}\right), 100.9\left(\mathrm{~d}, \mathrm{C}_{2},{ }^{1} \mathrm{~J}_{\mathrm{CF}} 185.1 \mathrm{~Hz}\right)$, $111.4\left(\mathrm{C}_{5}\right), 129.8\left(\mathrm{C}_{\mathrm{m}}\right), 130.8\left(\mathrm{C}_{\mathrm{o}}\right), 131.2\left(\mathrm{C}_{\mathrm{i}}\right), 134.6\left(\mathrm{C}_{\mathrm{p}}\right), 138.9\left(\mathrm{~d}, \mathrm{C}_{6},{ }^{3} J_{\mathrm{CF}} 3.7 \mathrm{~Hz}\right)$, $152.4\left(\mathrm{C}_{2}\right), 166.6\left(\mathrm{C}_{4}\right), 167.8(\mathrm{C}=\mathrm{O}) ; \stackrel{19}{\mathrm{~F} \mathrm{NMR}}\left(\mathrm{MeOH}-d_{4}, 282.4 \mathrm{MHz}\right) \delta-188.93$ 
MS (ESI $\left.{ }^{+}, \mathrm{m} / \mathrm{z}\right): 365\left[(\mathrm{M}+\mathrm{H})^{+}, 100 \%\right]$; Anal. Calcd. (\%) for $\mathrm{C}_{17} \mathrm{H}_{17} \mathrm{FN}_{2} \mathrm{O}_{6}: \mathrm{C}, 56.04$; H, 4.7; N, 7.69; O, 26.35; F, 5.21. Found: C, 56.0; H, 4.6; N, 7.7; O, 26.3; F, 5.3. 
5'-O-Levulinyl- $\alpha$-D-thymidine

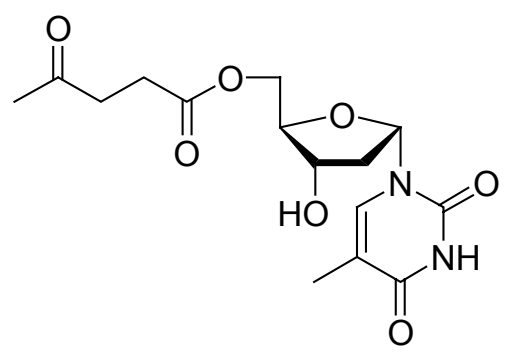

$2 a$

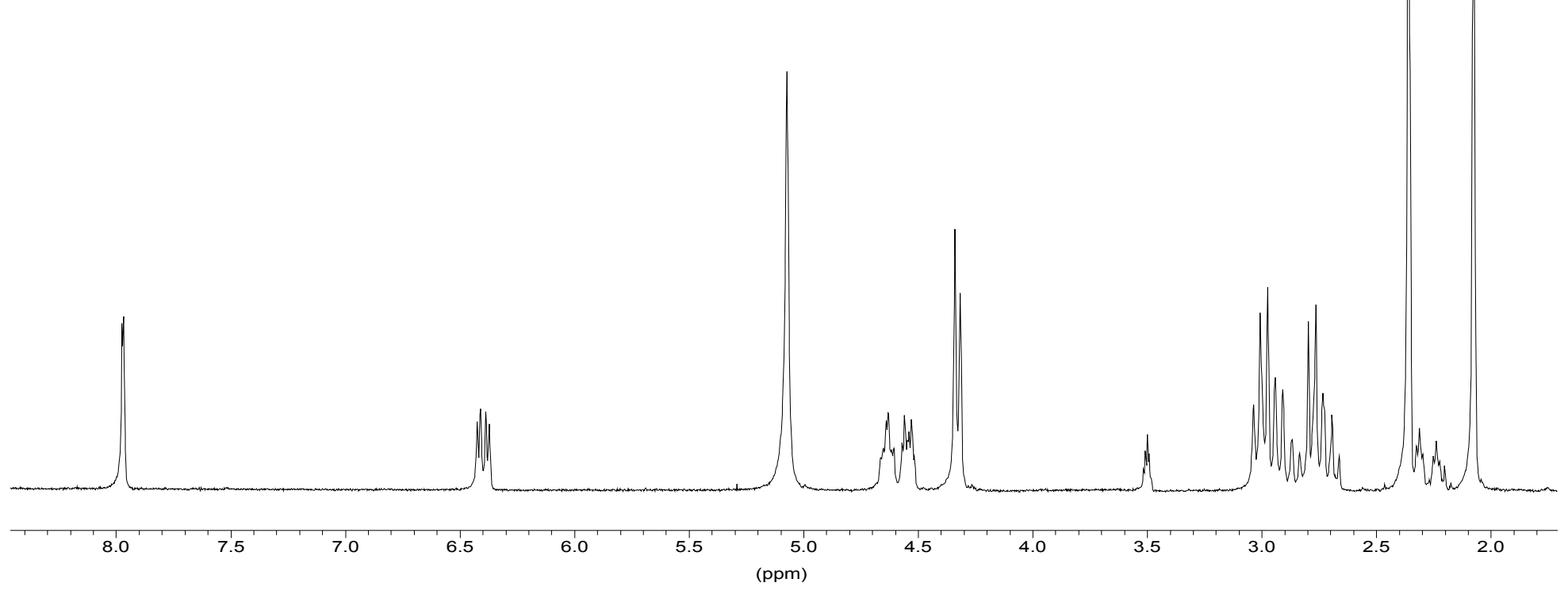


5'-O-Levulinyl- $\alpha$-D-thymidine

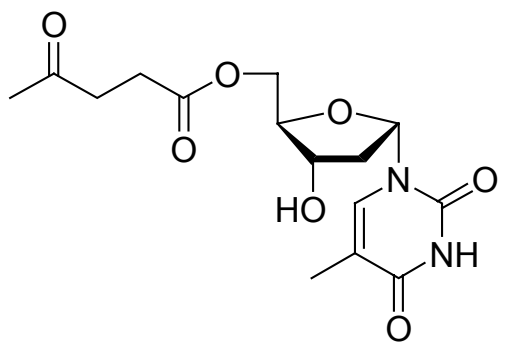

$2 a$ 


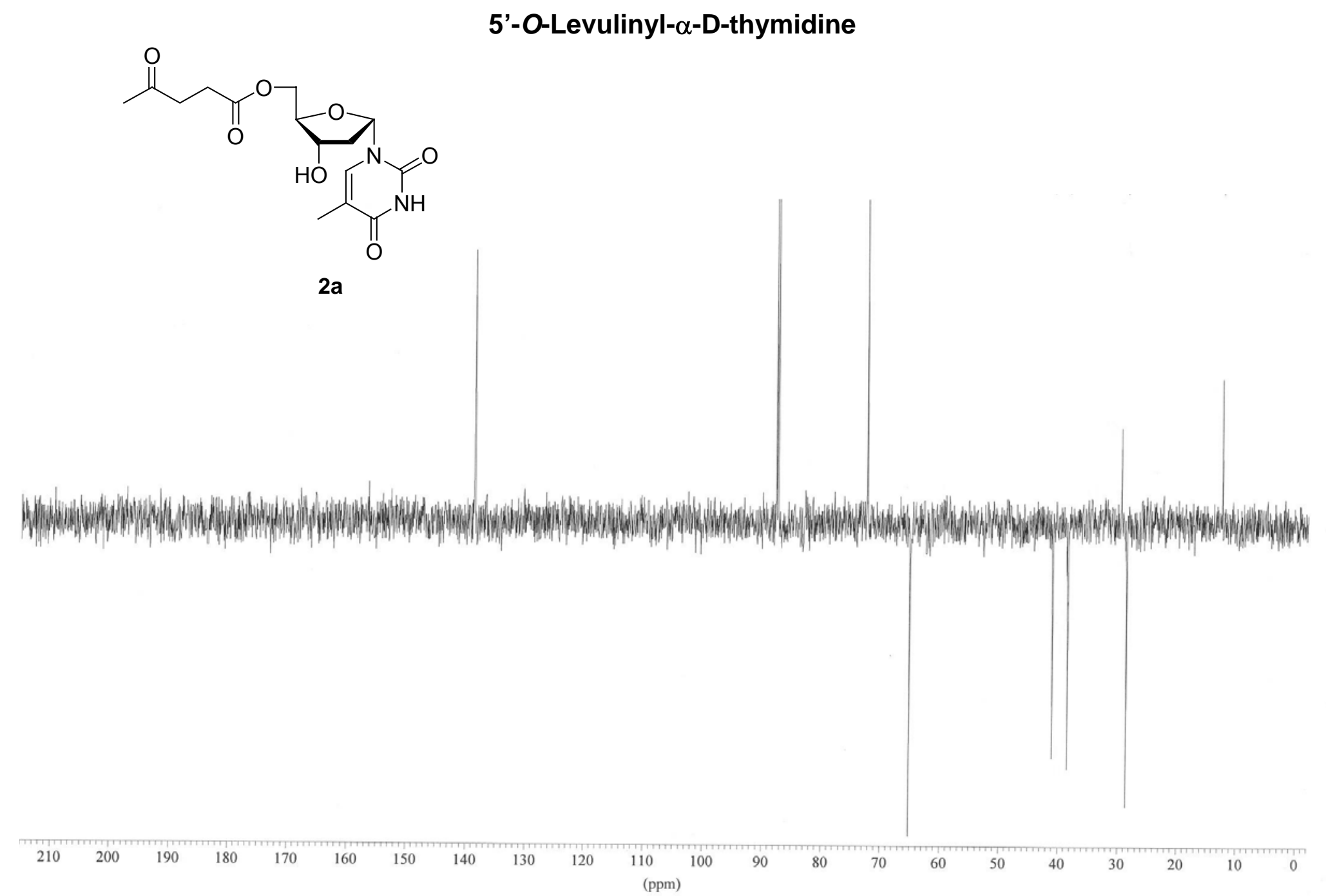


N-Benzoyl-5'-O-levulinyl- $\alpha$-D-2'-deoxycytidine

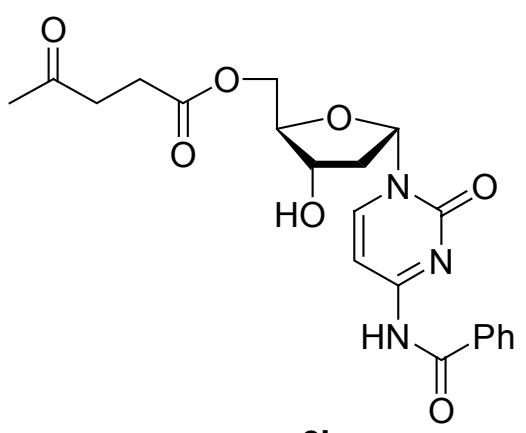

$2 b$

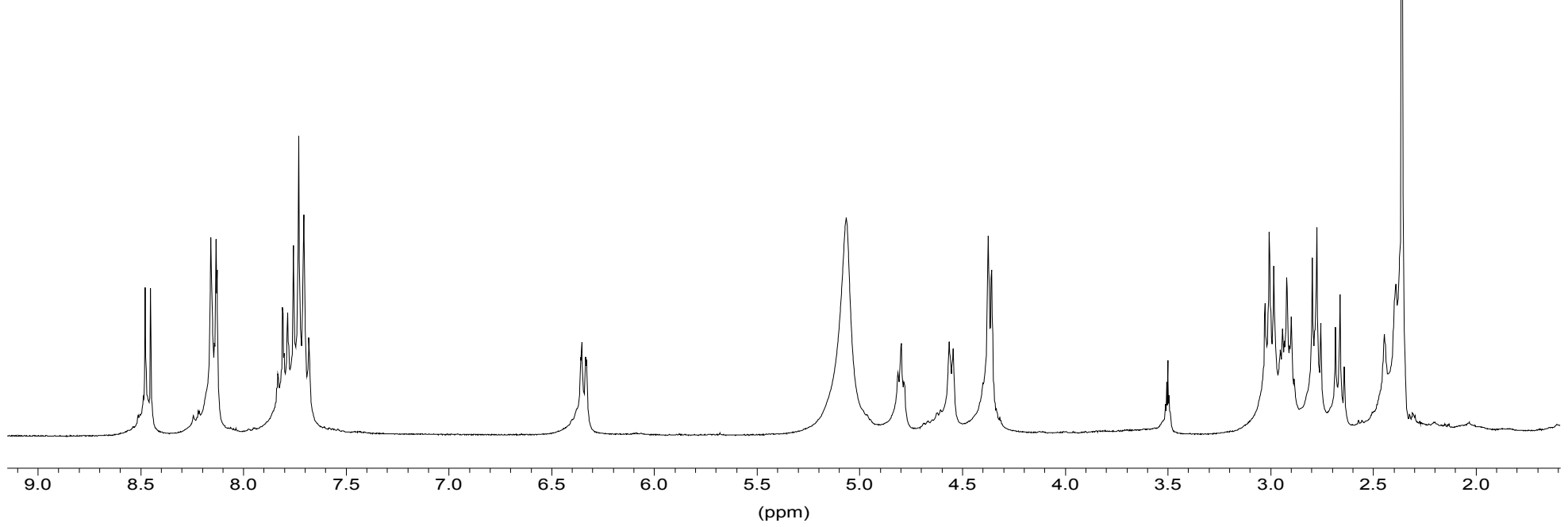




\section{N-Benzoyl-5'-O-levulinyl- $\alpha$-D-2'-deoxycytidine}

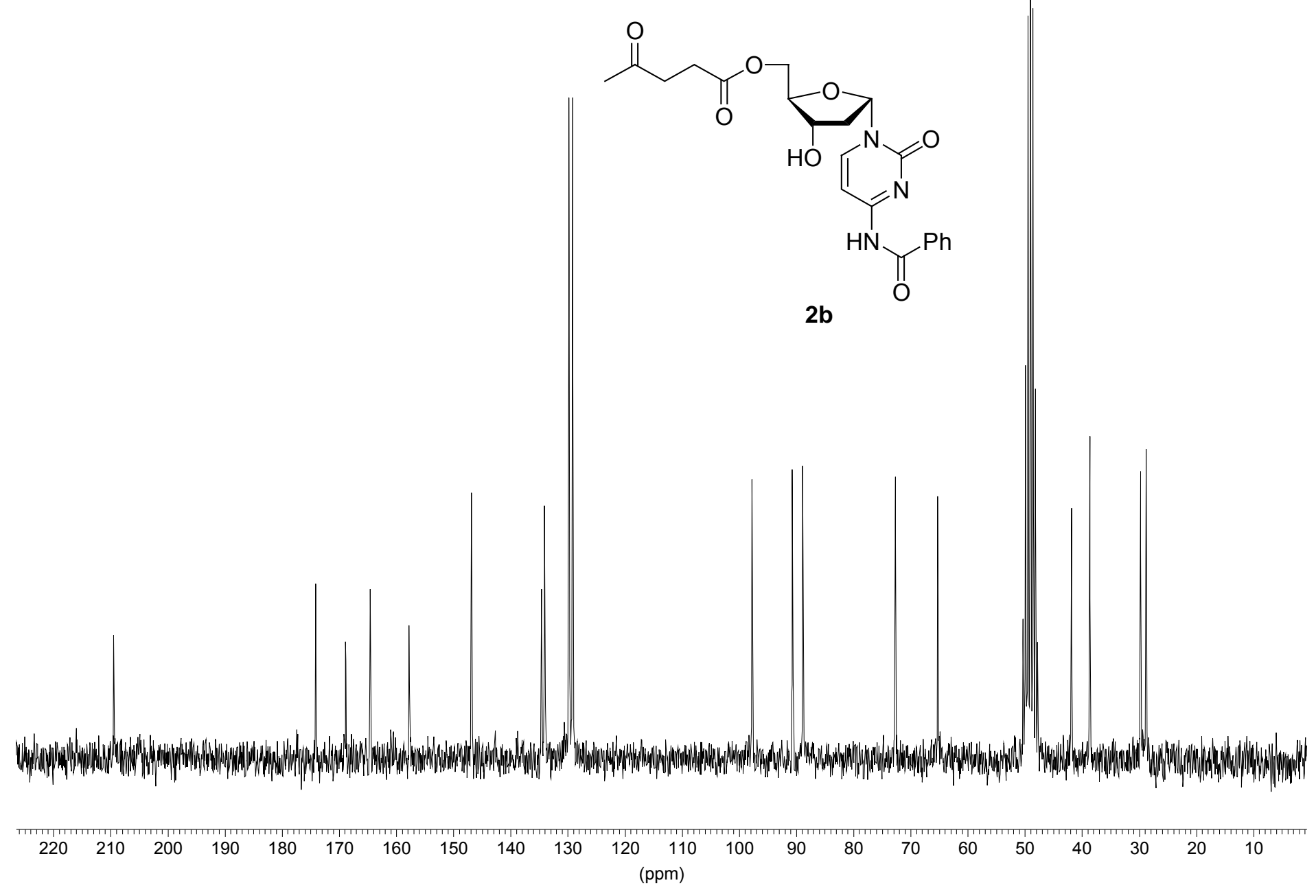




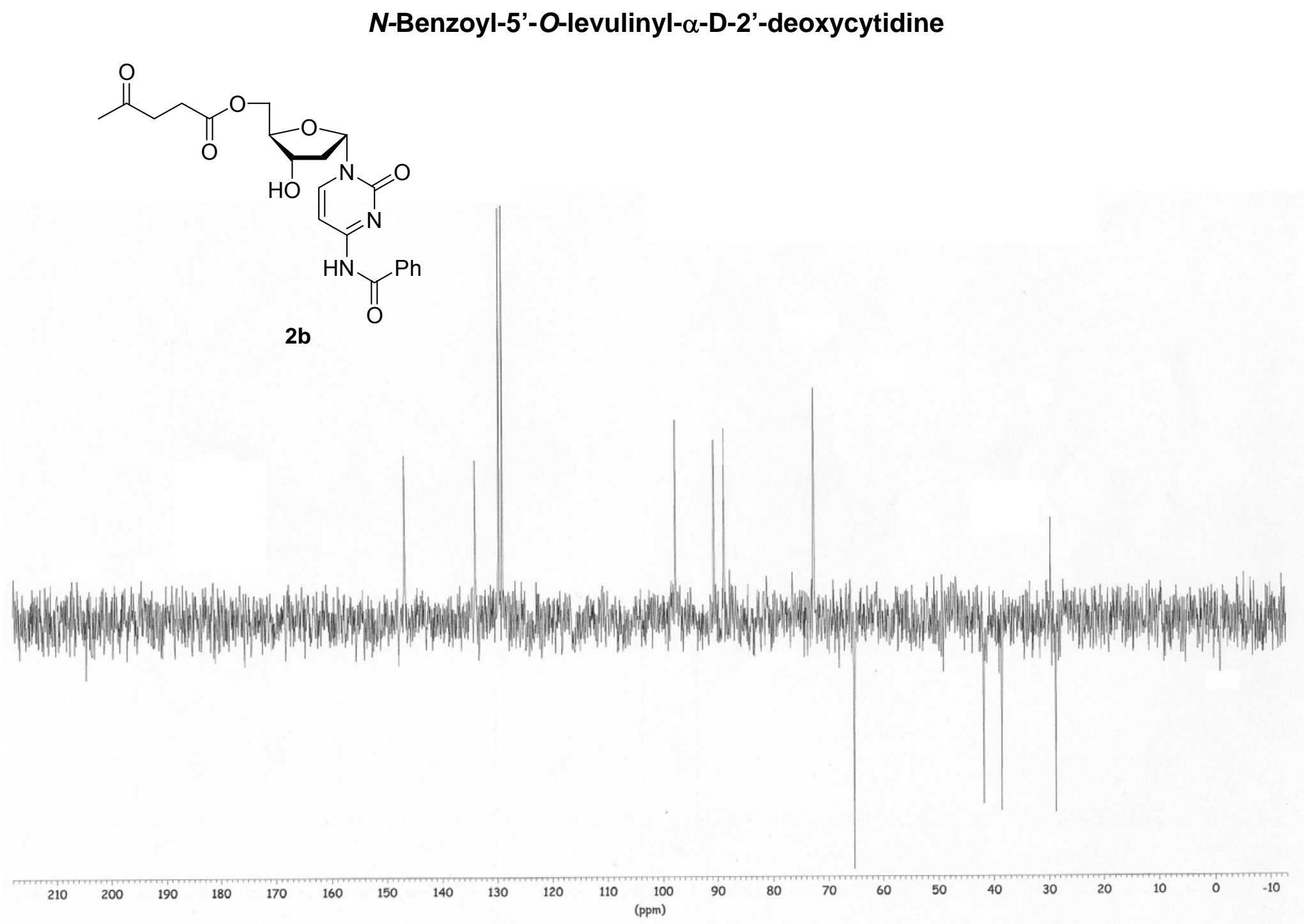


$N$-Benzoyl-5'-O-levulinyl- $\alpha$-D-2'-deoxyadenosine

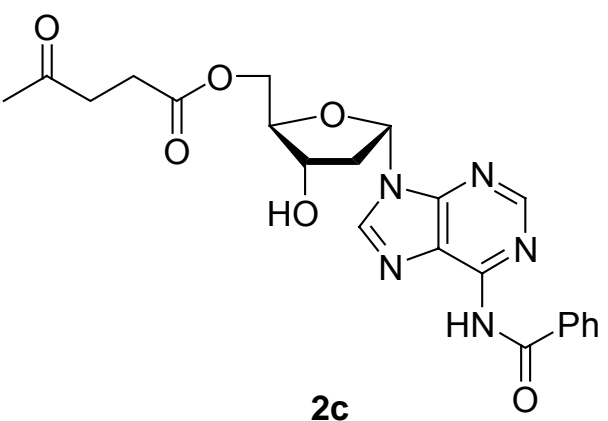

2c

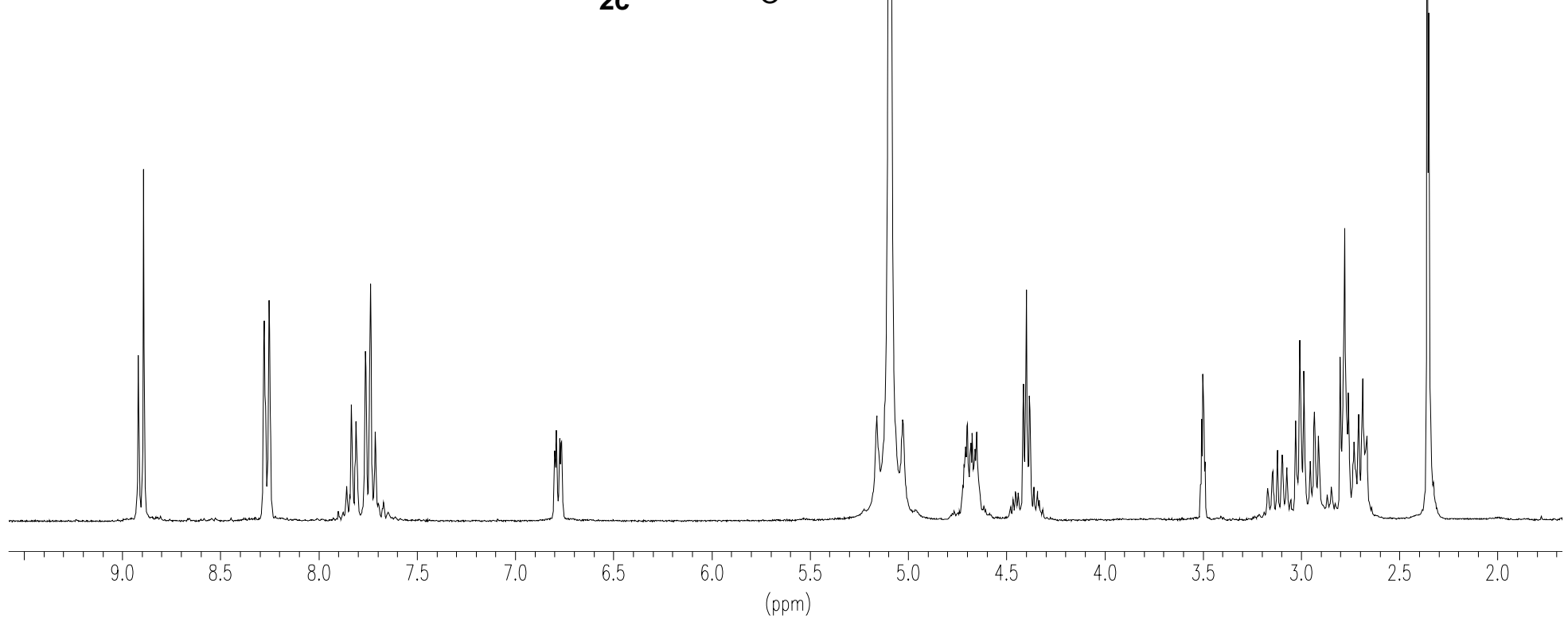


N-Benzoyl-5'-O-levulinyl- $\alpha-D-2$ '-deoxyadenosine

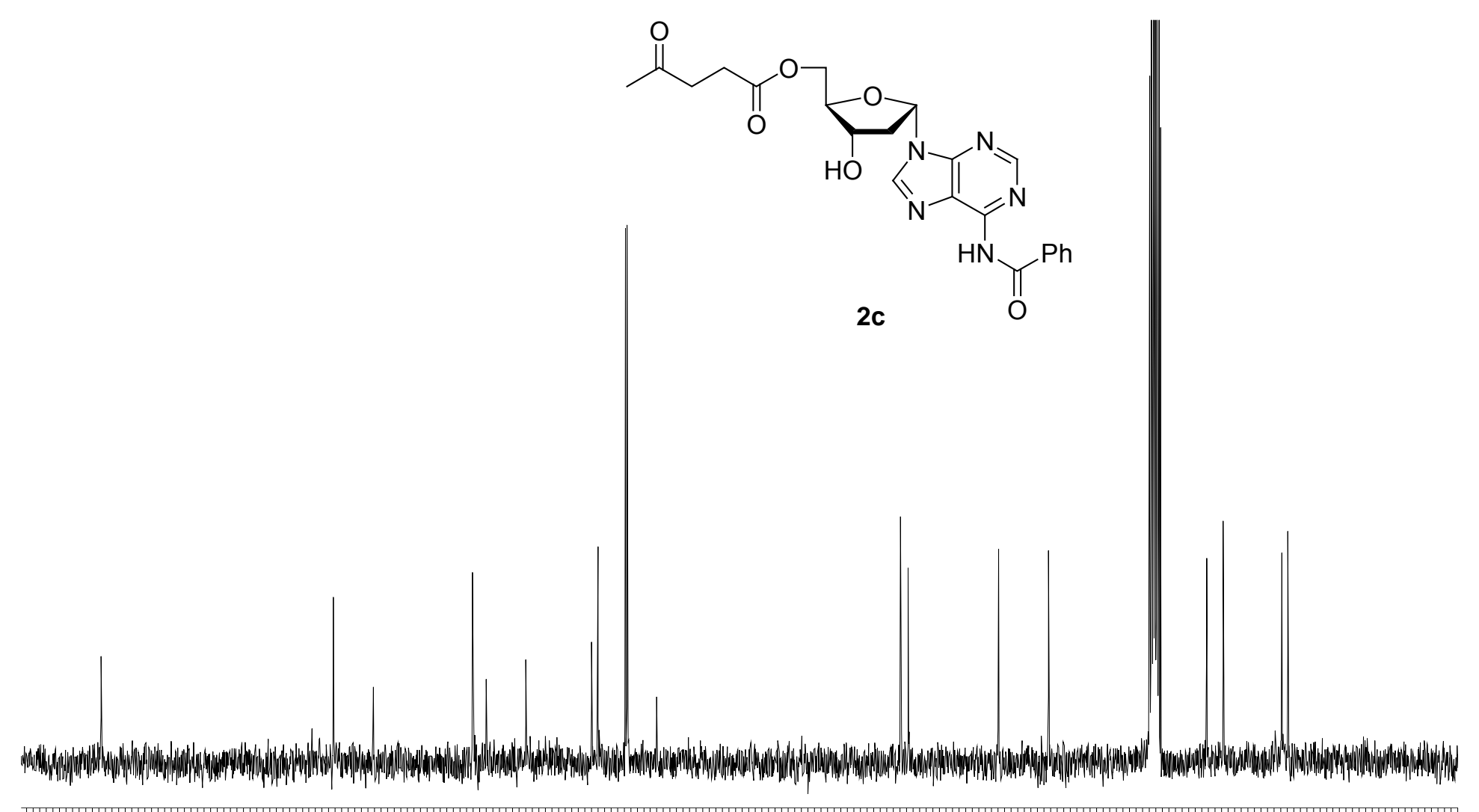


N-Benzoyl-5'-O-levulinyl- $\alpha-D-2$ '-deoxyadenosine

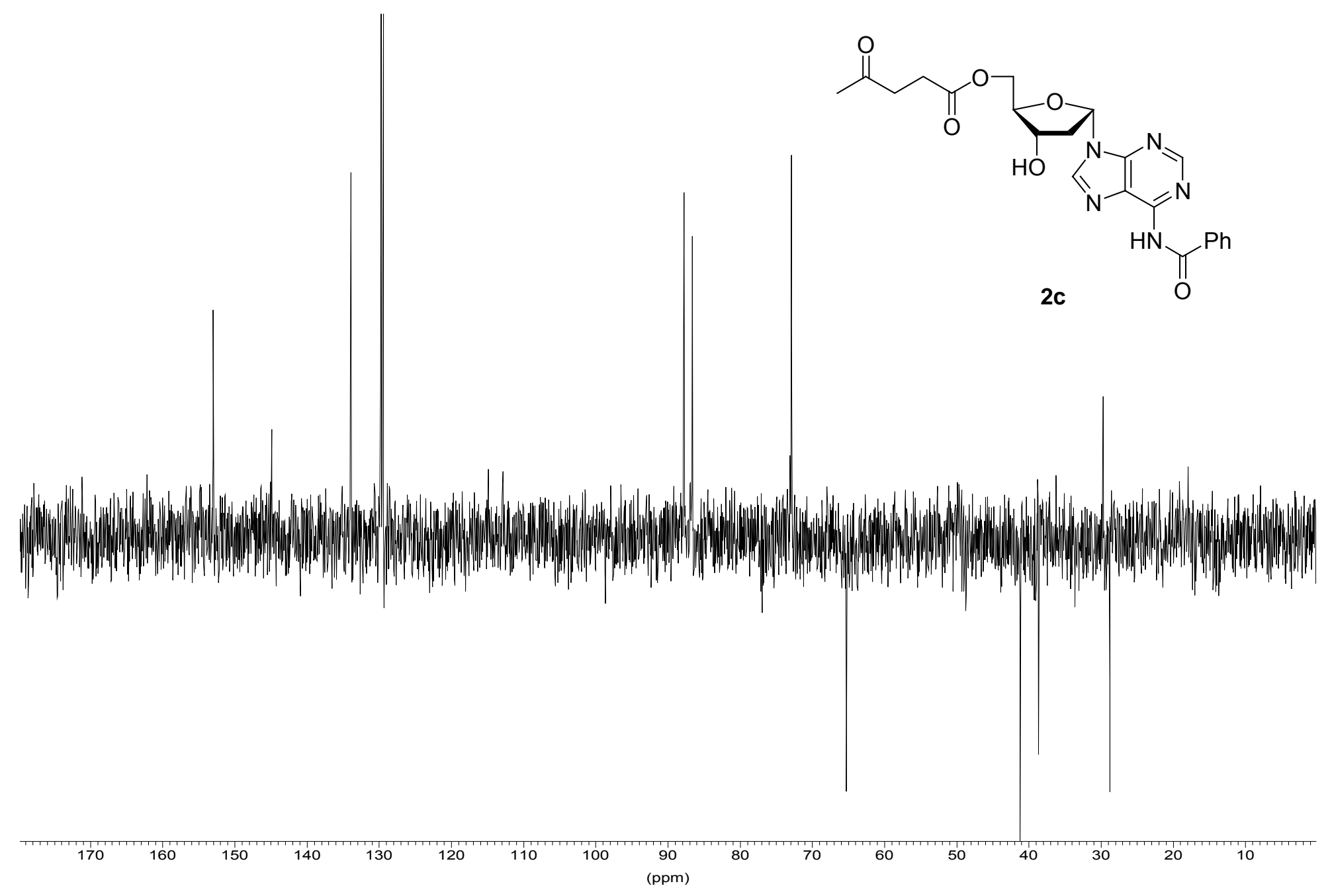


$N$-Isobutyryl-5'-O-levulinyl- $\alpha-D-2$ '-deoxyguanosine

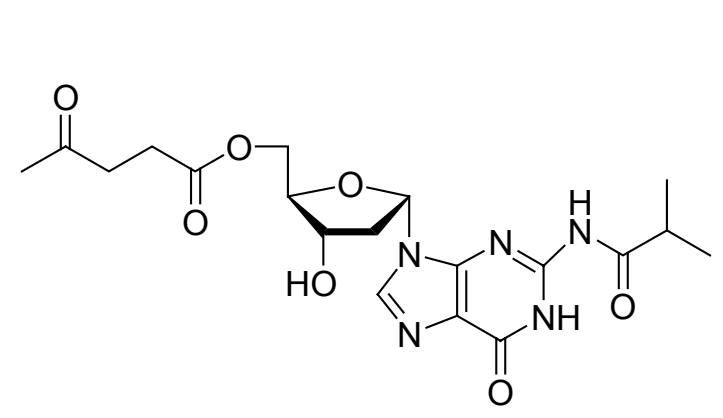

$2 d$ 
$N$-Isobutyryl-5'-O-levulinyl- $\alpha-D-2$ '-deoxyguanosine

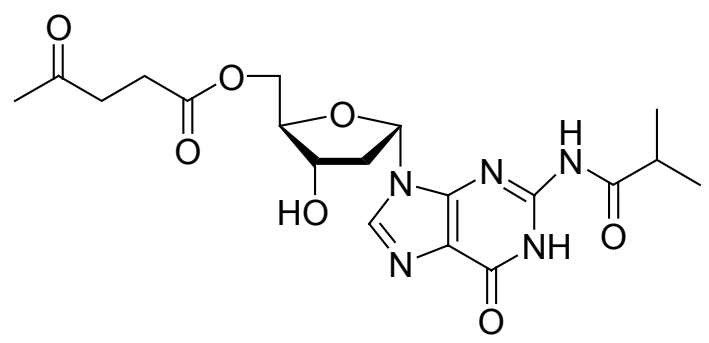

2d
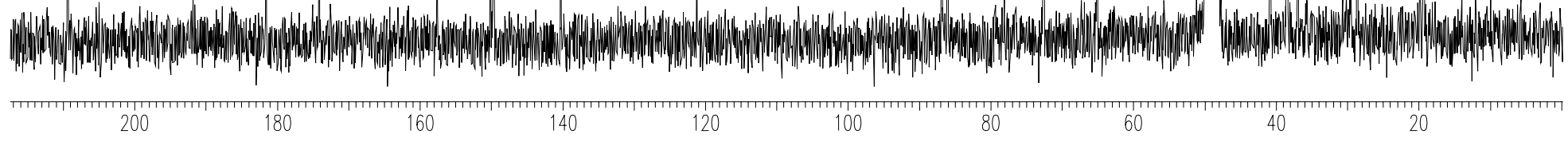
$N$-Isobutyryl-5'-O-levulinyl- $\alpha$-D-2'-deoxyguanosine

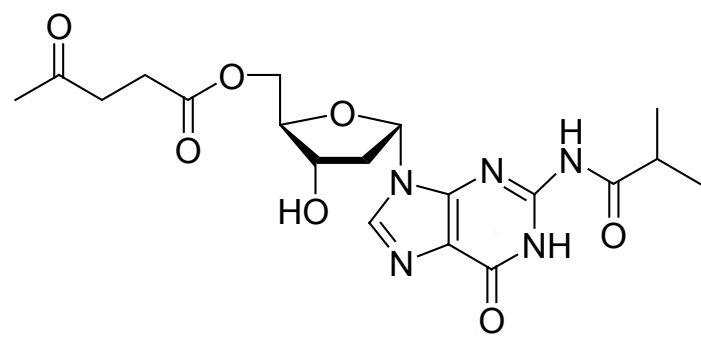

2d

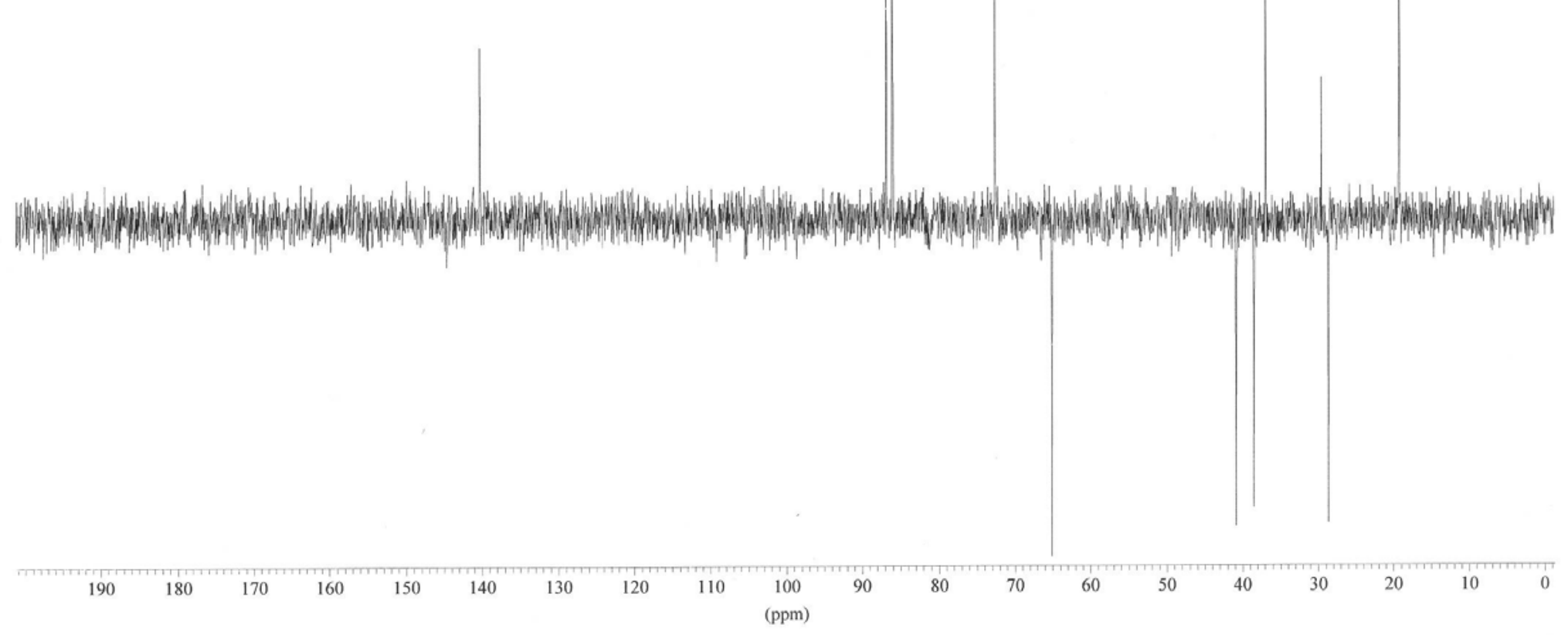


3',5'-Di-O-levulinyl- $\alpha$-D-thymidine

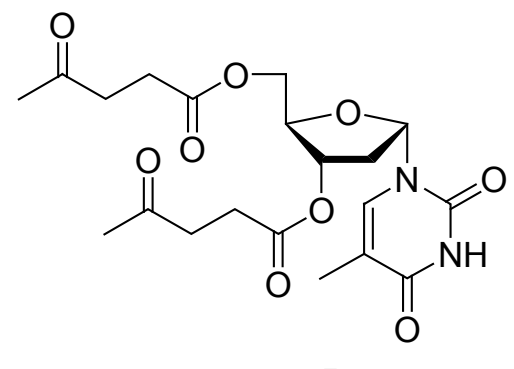

$5 a$ 
3',5'-Di-O-levulinyl- $\alpha$-D-thymidine

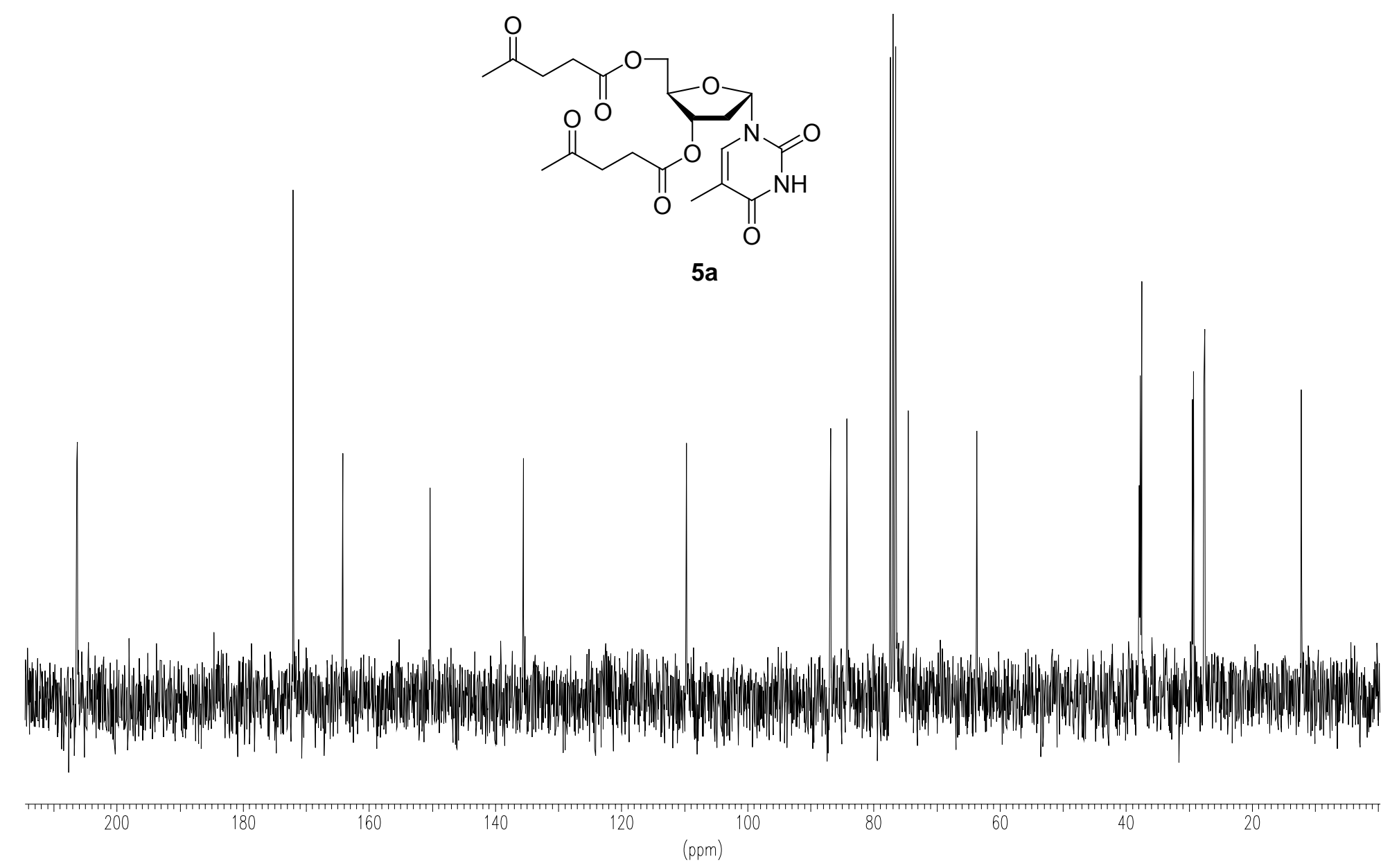


3',5'-Di-O-levulinyl- $\alpha$-D-thymidine

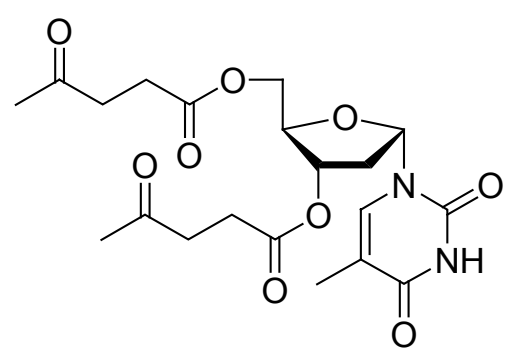

$5 \mathbf{a}$ 
N-Benzoyl-3',5'-di-O-levulinyl- $\alpha-D-2$ '-deoxycytidine

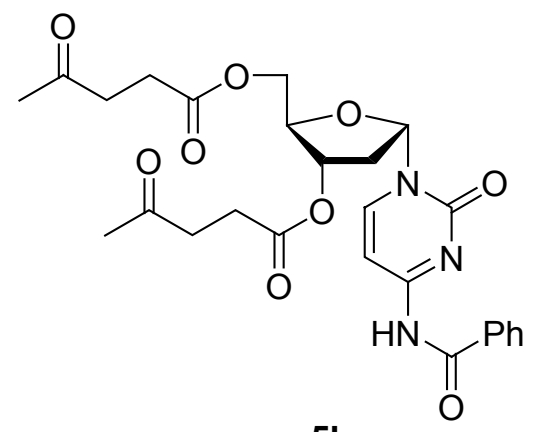

$5 b$ 
N-Benzoyl-3',5'-di-O-levulinyl- $\alpha-D-2$ '-deoxycytidine

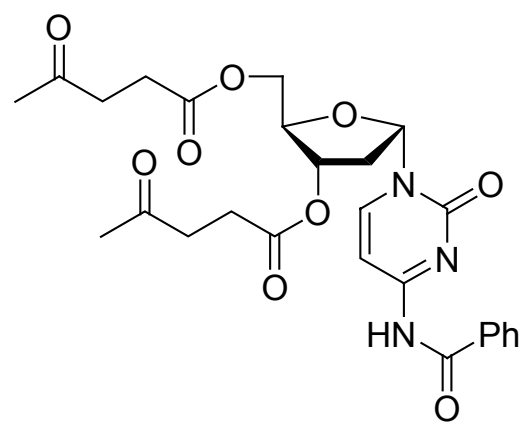

$5 b$

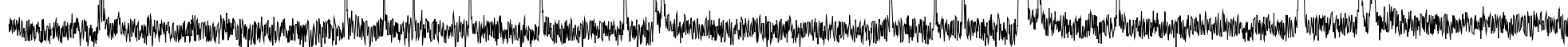


N-Benzoyl-3',5'-di-O-levulinyl- $\alpha-D-2$ '-deoxycytidine

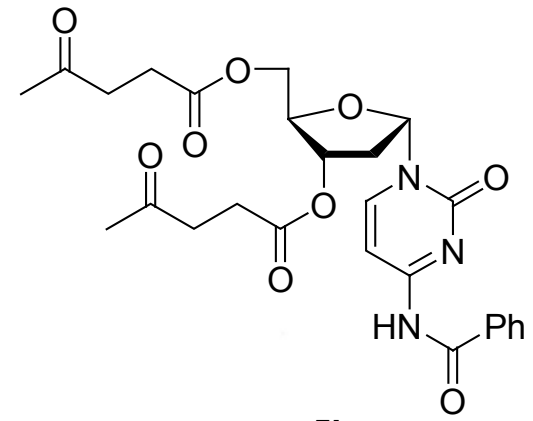

$5 b$

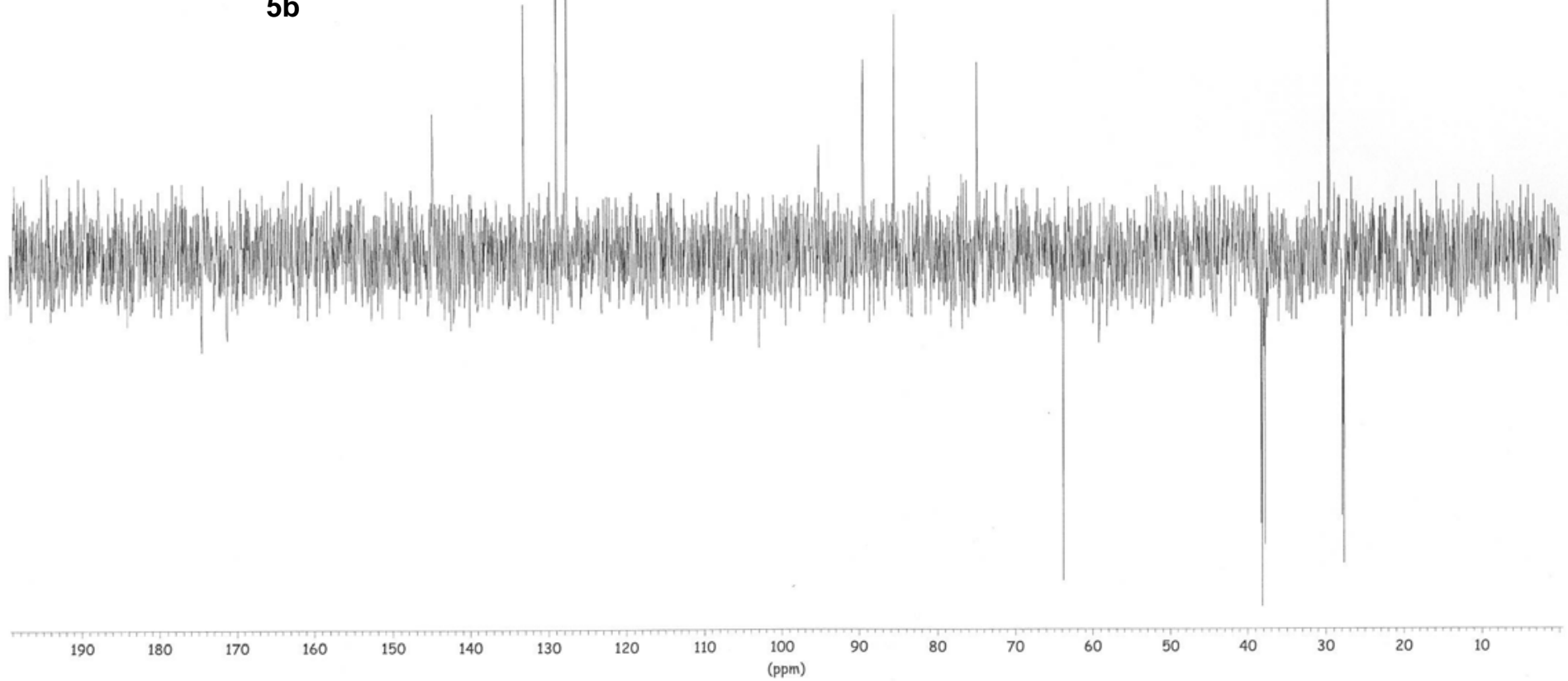


3'-O-Levulinyl- $\alpha$-D-thymidine

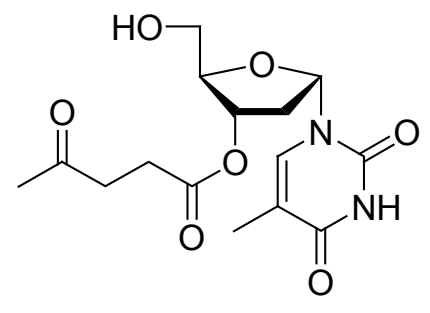

$6 a$ 
3'-O-Levulinyl- $\alpha$-D-thymidine

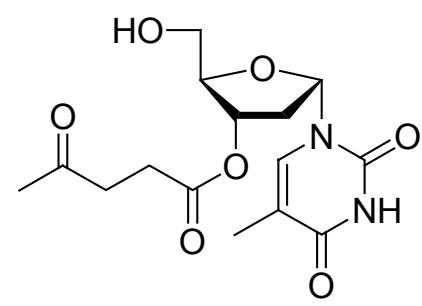

$6 a$

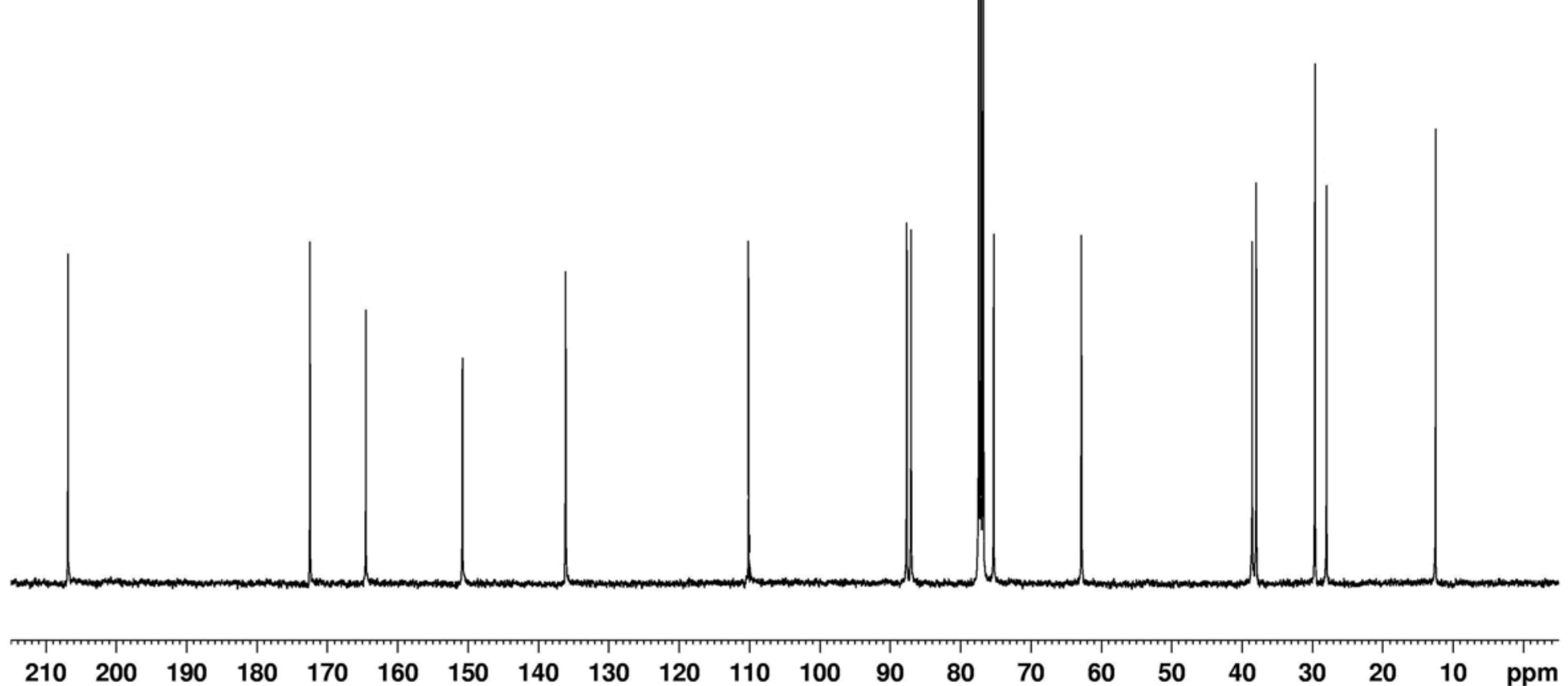


3'-O-Levulinyl- $\alpha$-D-thymidine

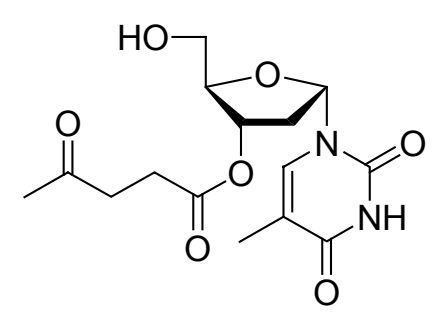

$6 a$

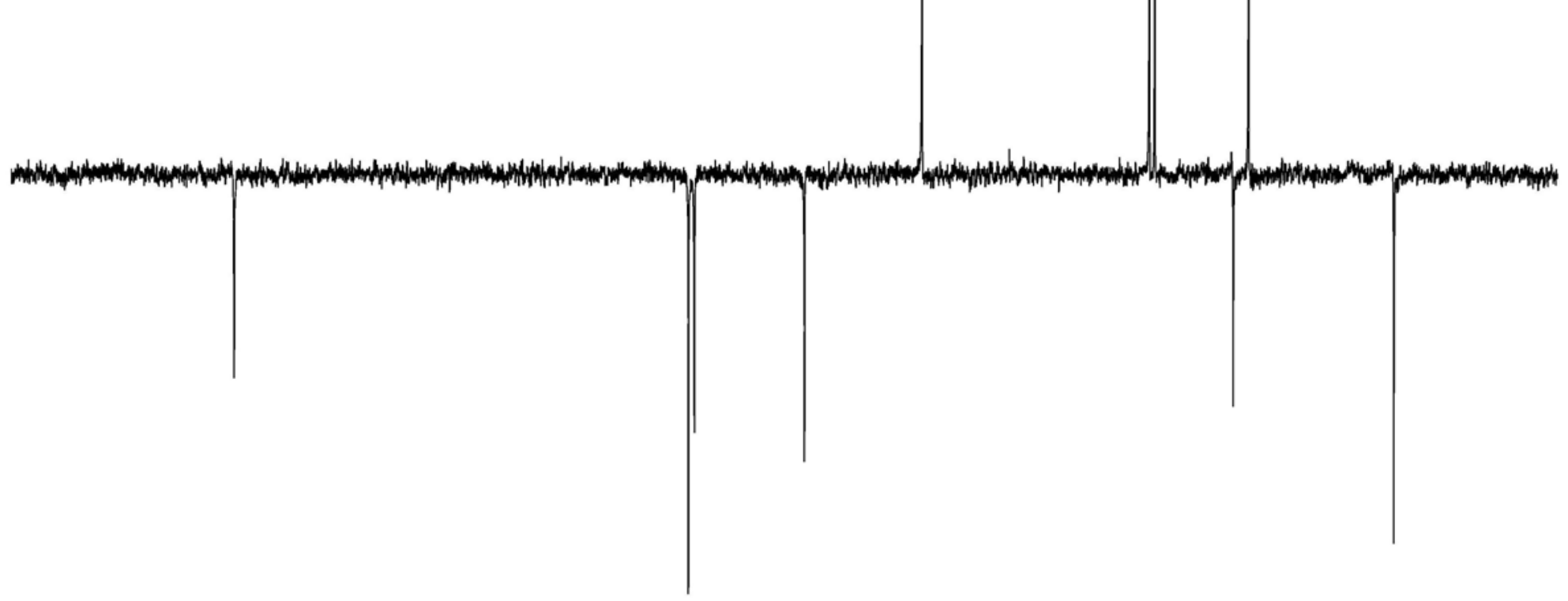




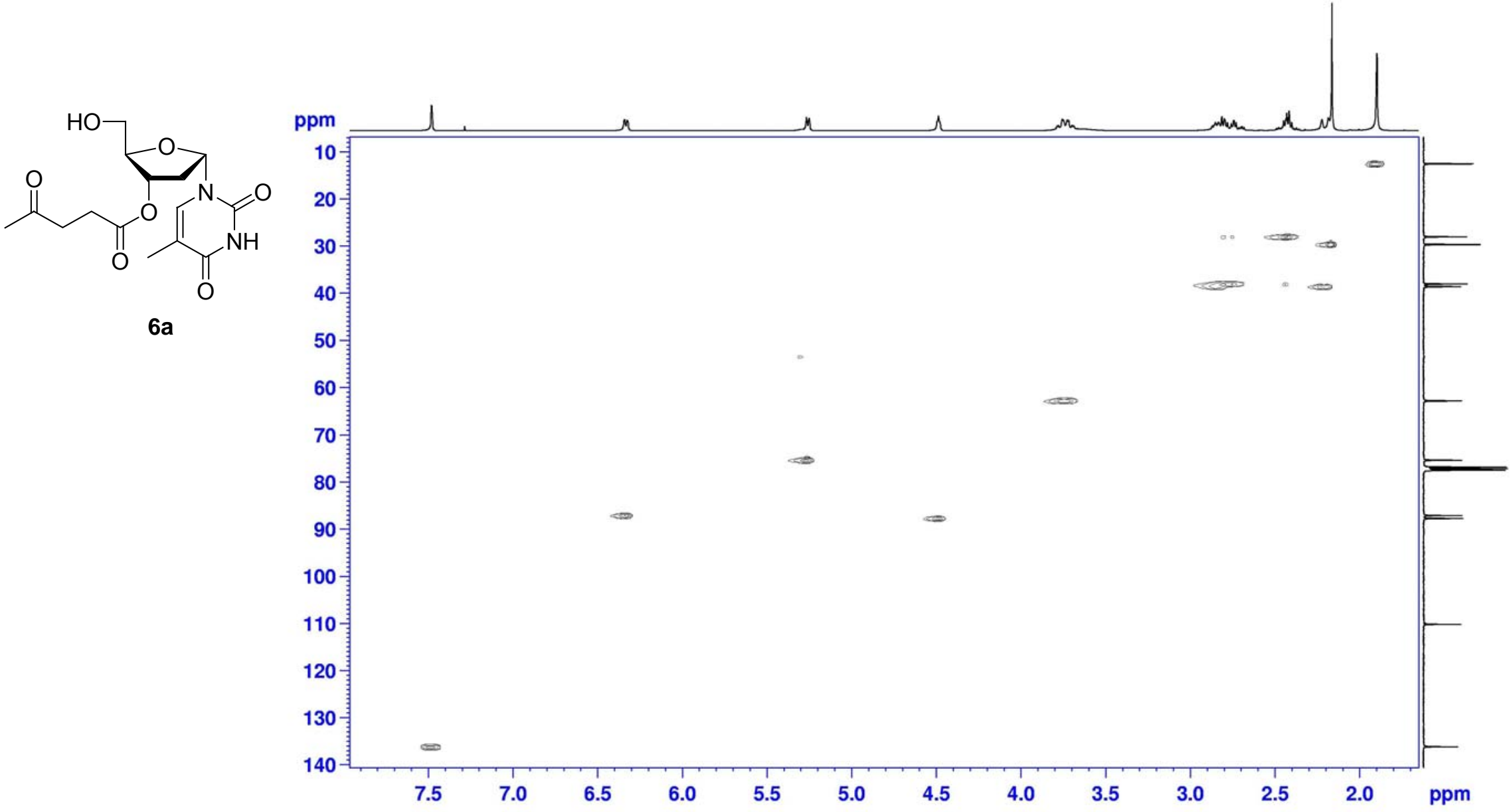




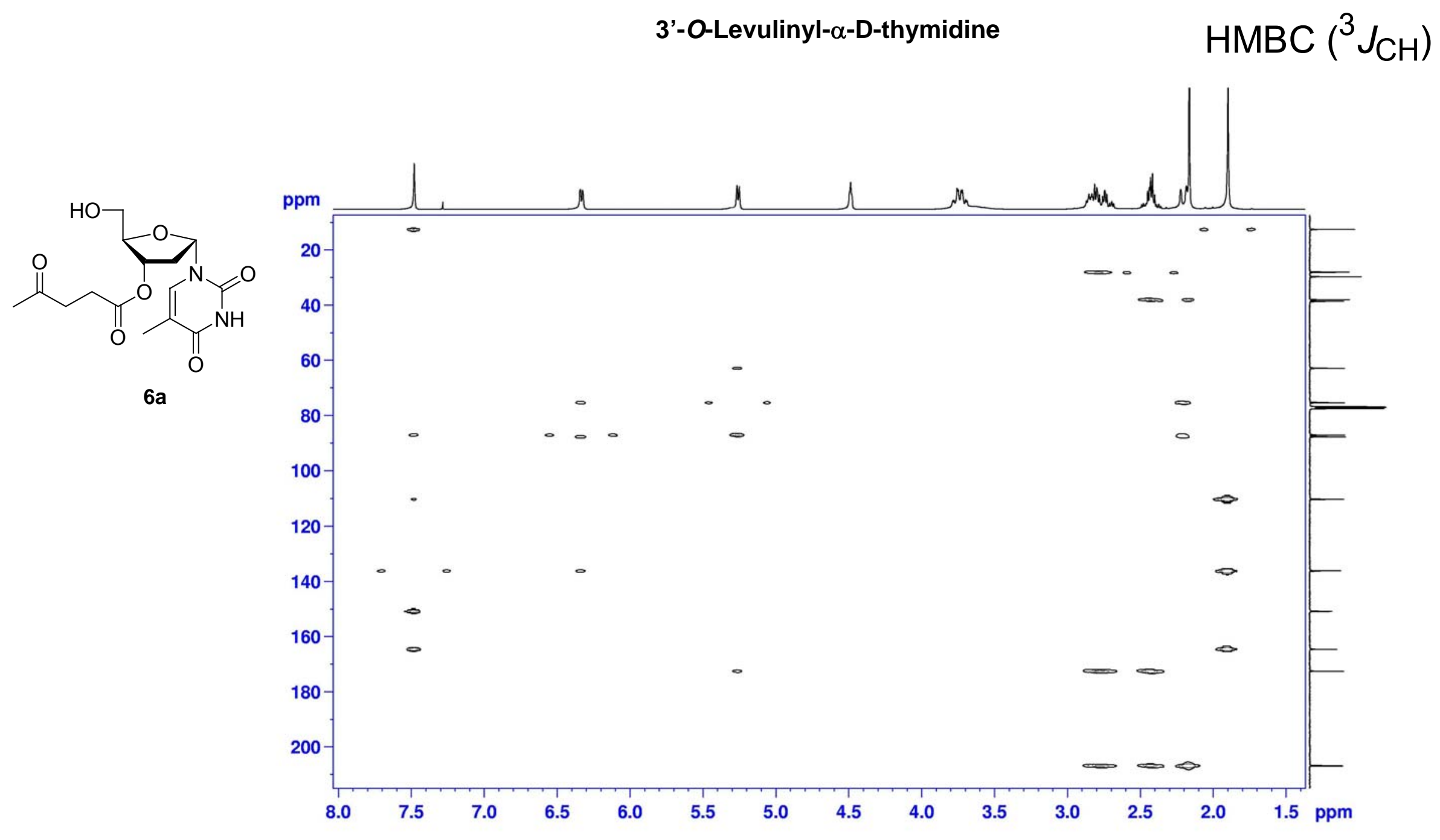




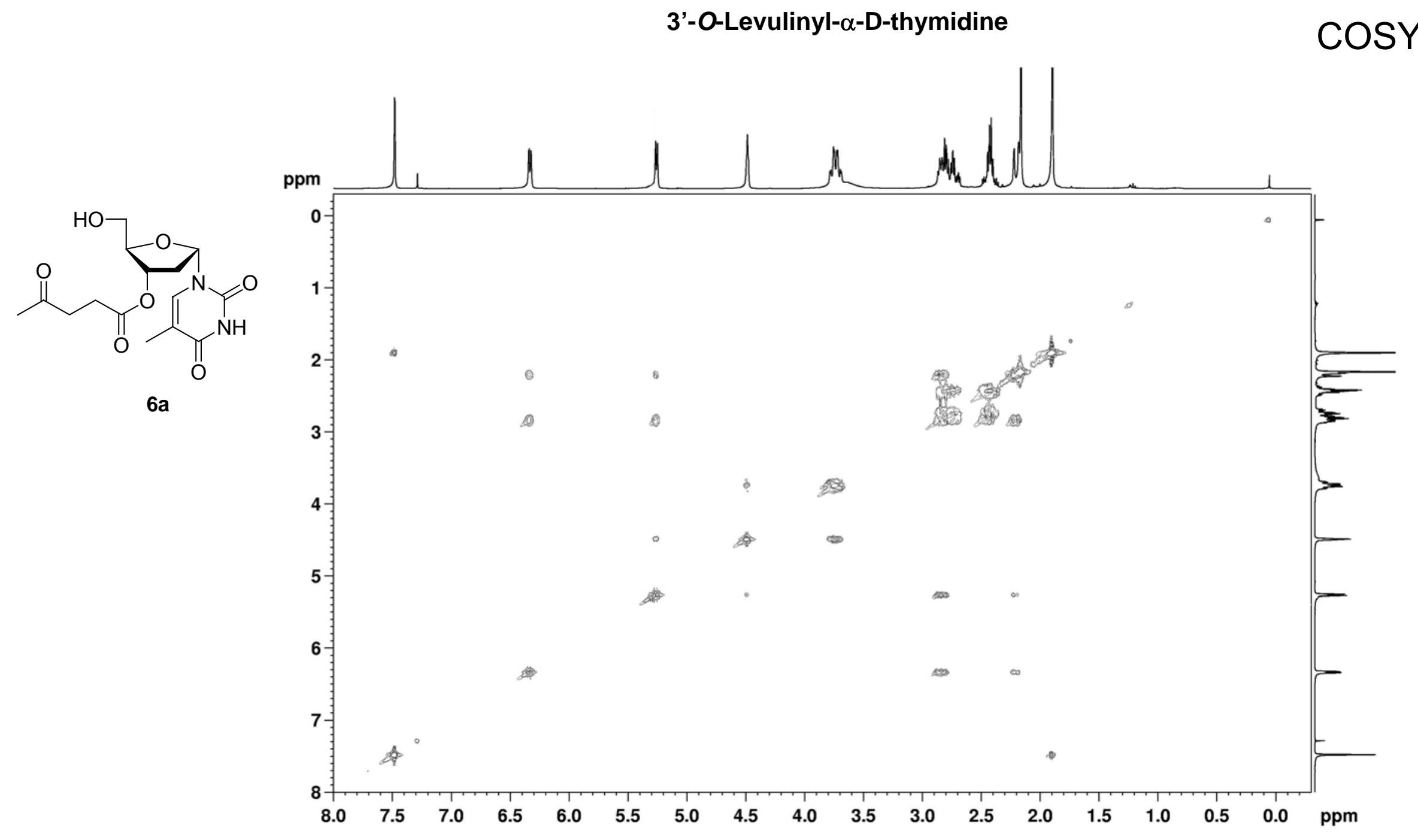




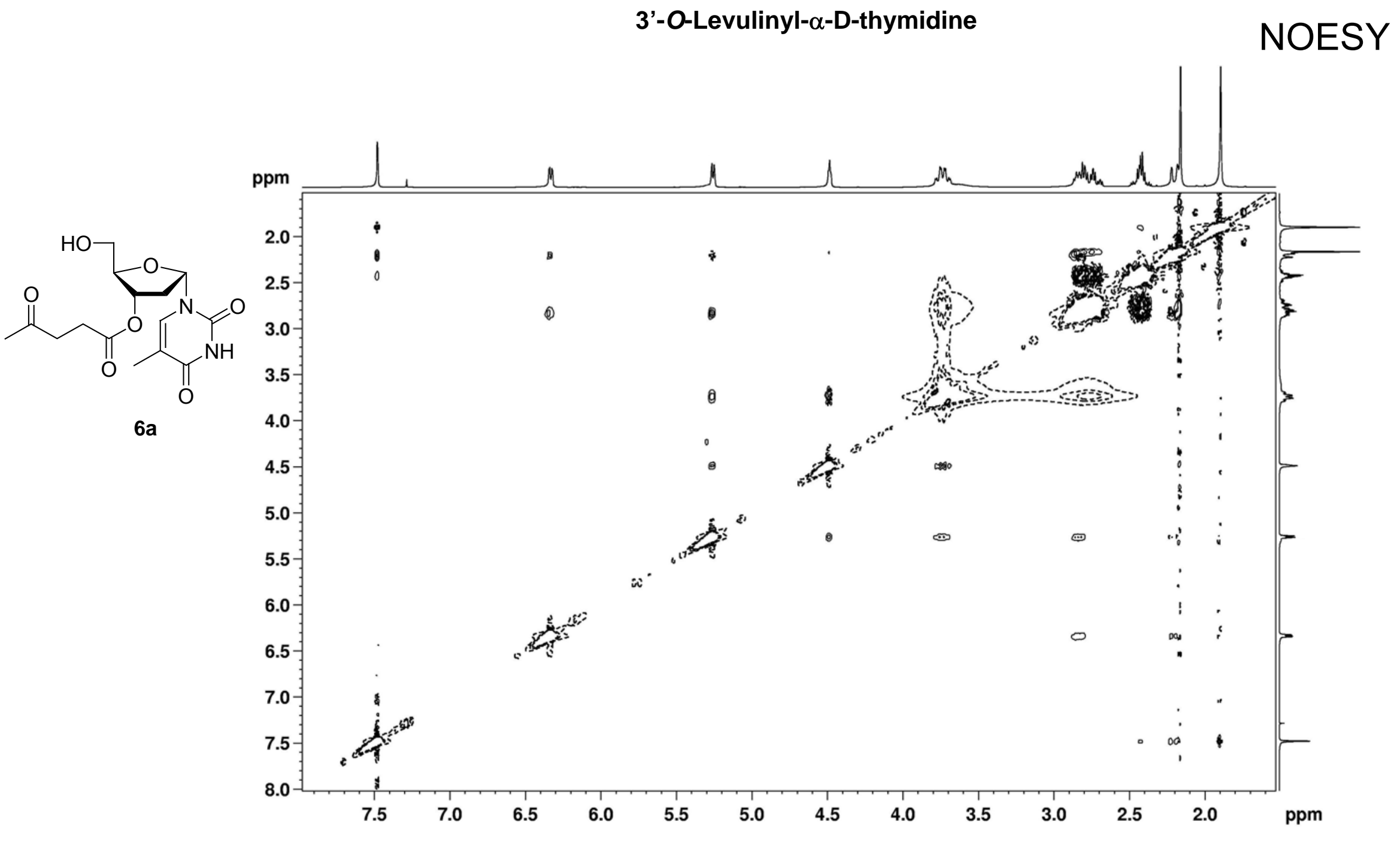


N-Benzoyl-3'-O-levulinyl- $\alpha$-D-2'-deoxycytidine

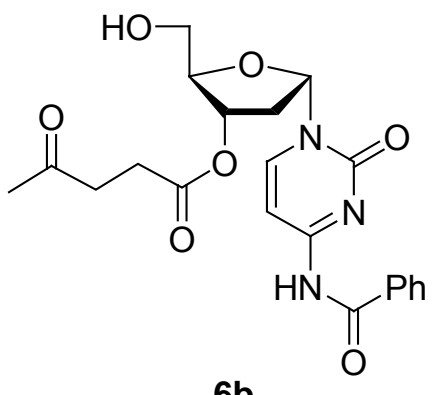

6b 
N-Benzoyl-3'-O-levulinyl- $\alpha$-D-2'-deoxycytidine

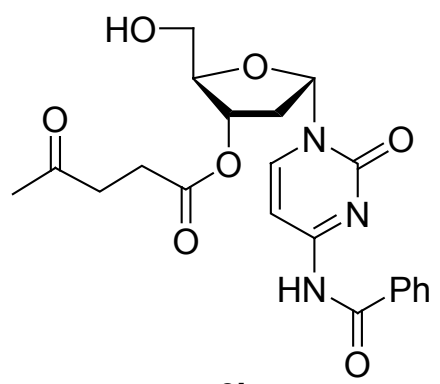

$6 b$ 


\section{N-Benzoyl-3'-O-levulinyl- $\alpha$-D-2'-deoxycytidine}

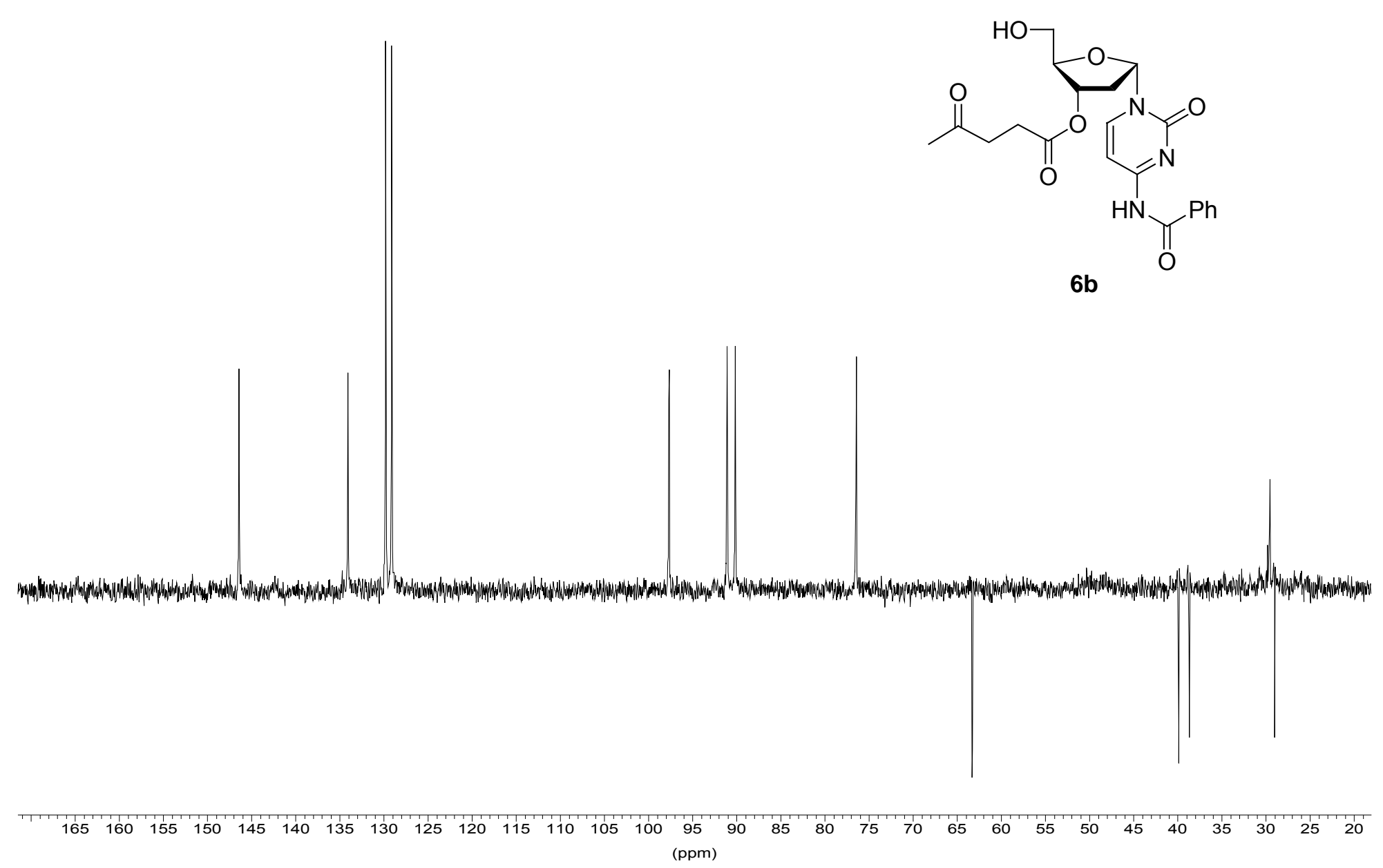


3',5'-Di-O-(p-chlorobenzoyl)- $\beta$-D-thymidine

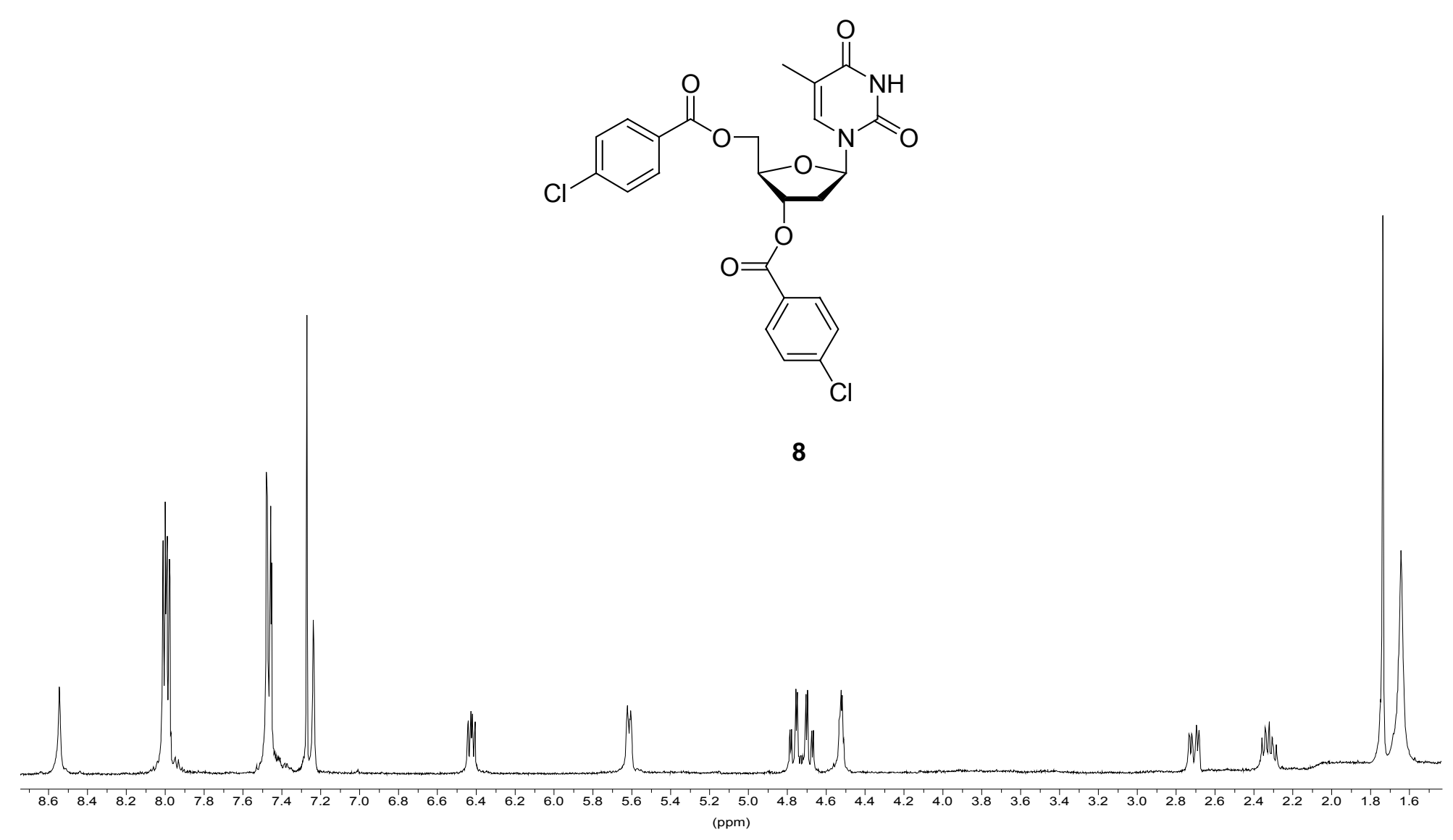


3',5'-Di-O-(p-chlorobenzoyl)- $\beta$-D-thymidine

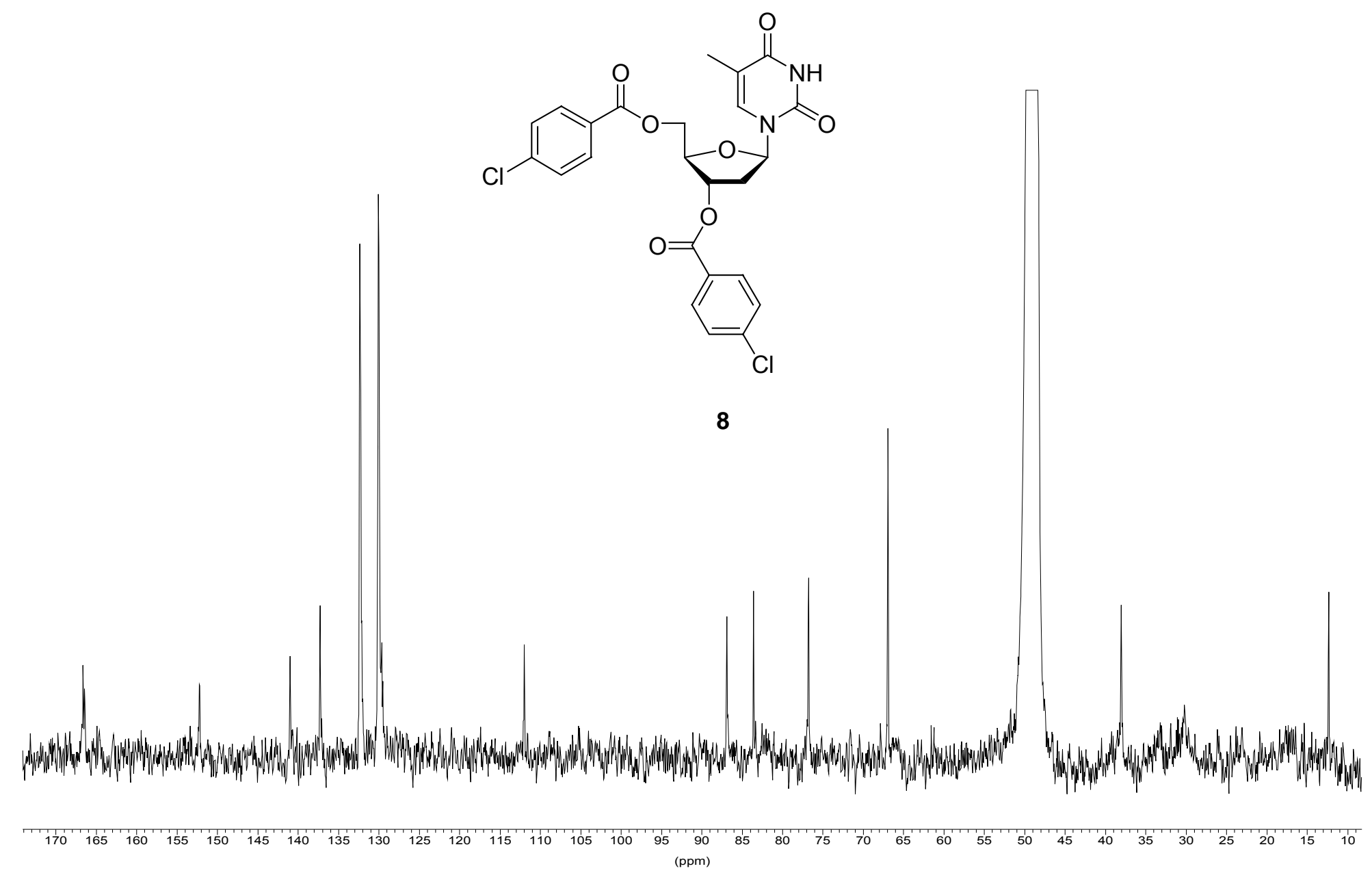


3',5'-Di-O-(p-chlorobenzoyl)- $\beta$-D-thymidine

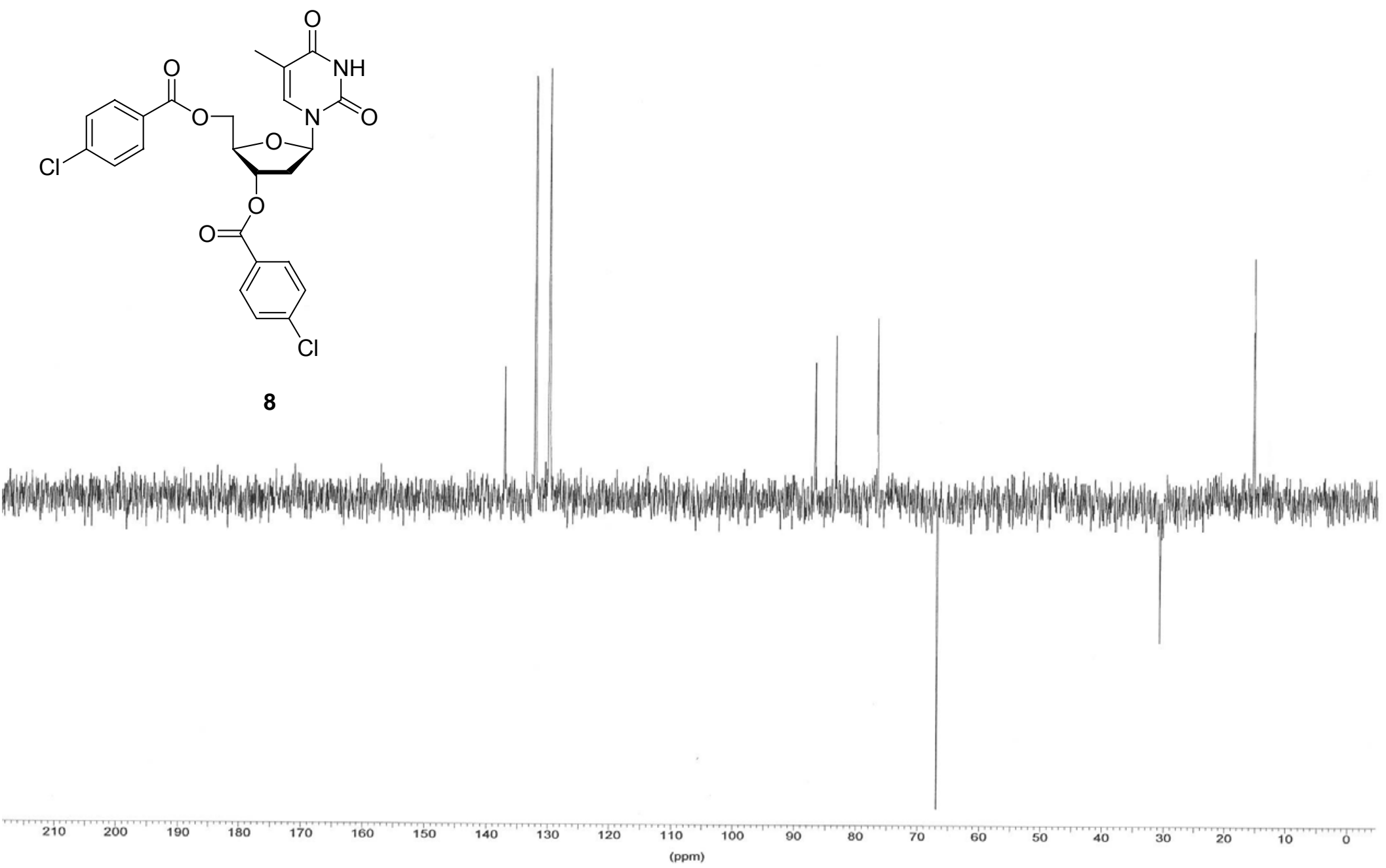


3'-O-(p-chlorobenzoyl)- $\alpha$-D-thymidine

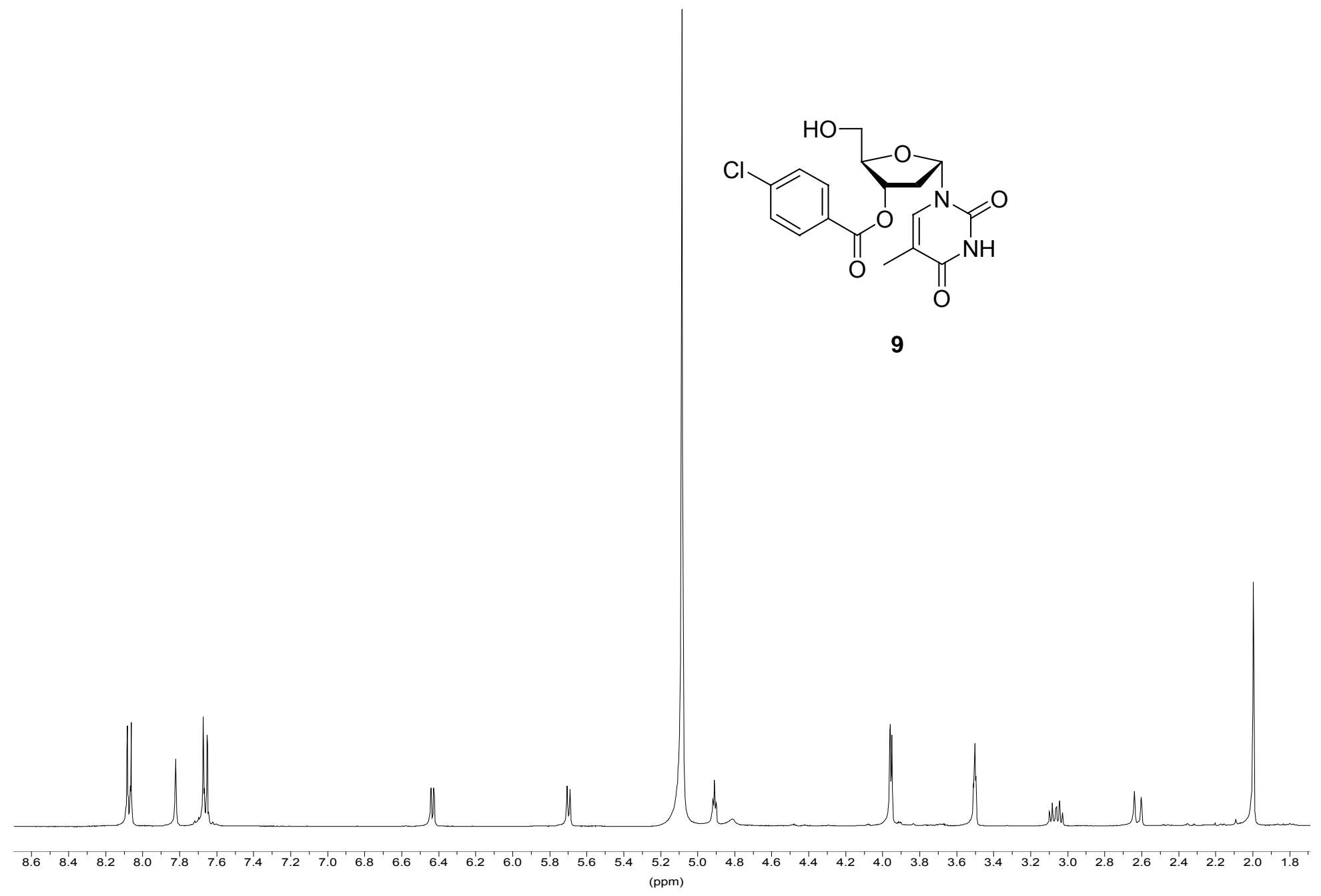


3'-O-(p-chlorobenzoyl)- $\alpha$-D-thymidine

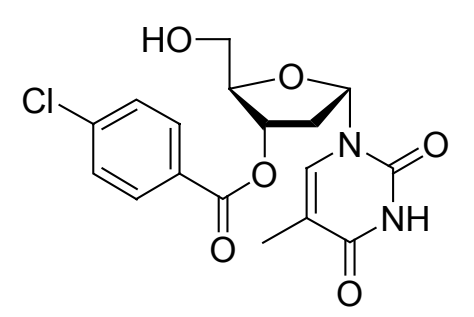

9

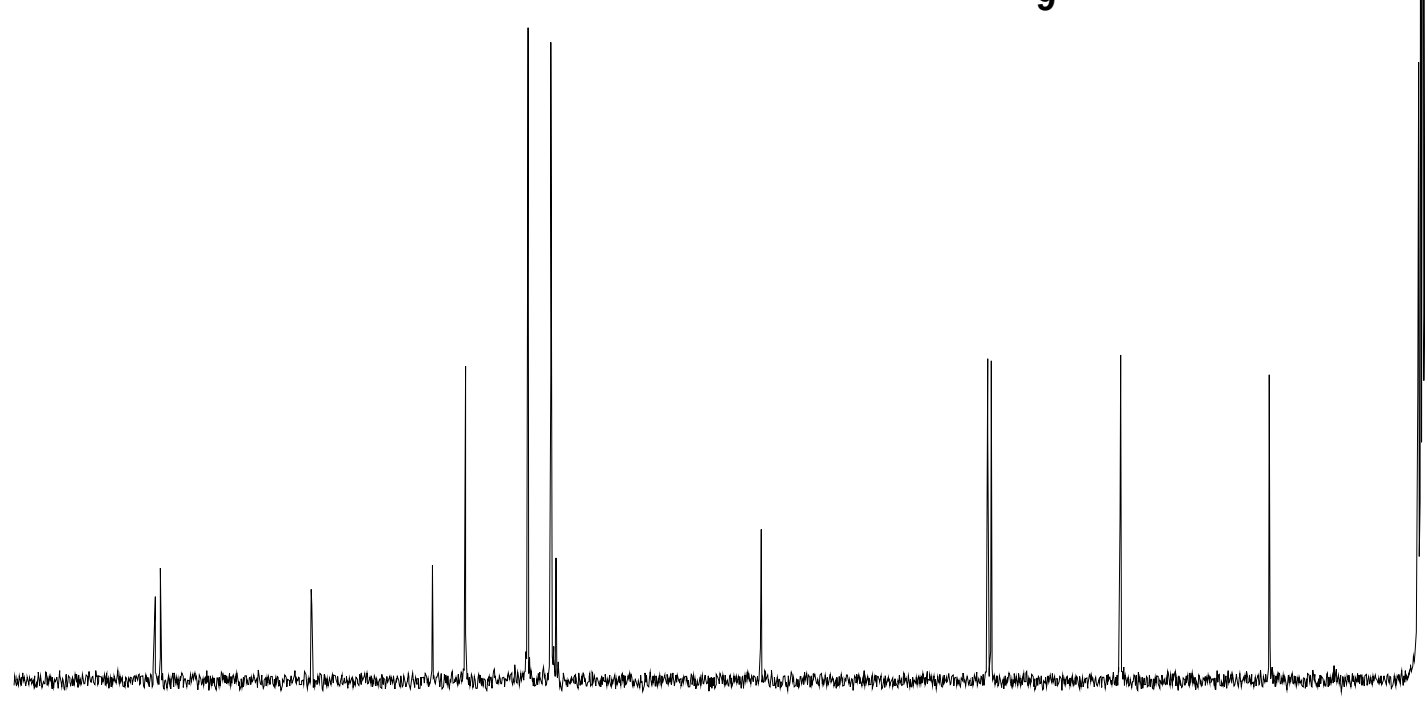

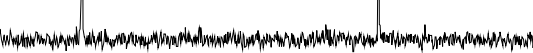

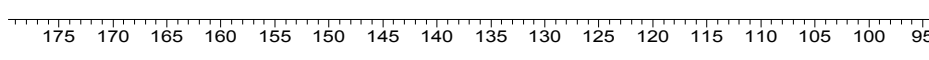
(ppm) 


\section{3'-O-(p-chlorobenzoyl)- $\alpha-D-$ thymidine}

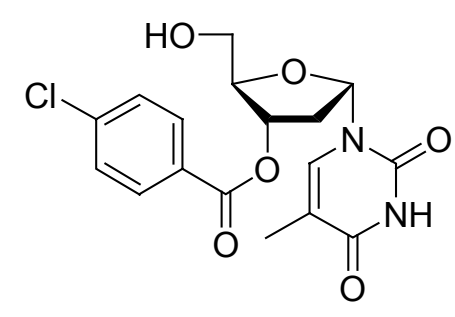

9

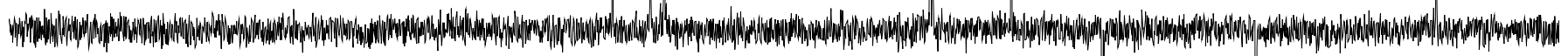


1-(3',5'-Di-O-Benzoyl-2'-Fluoro-2'-deoxy- $\alpha / \beta$-D-Arabinofuranosyl)Thymine

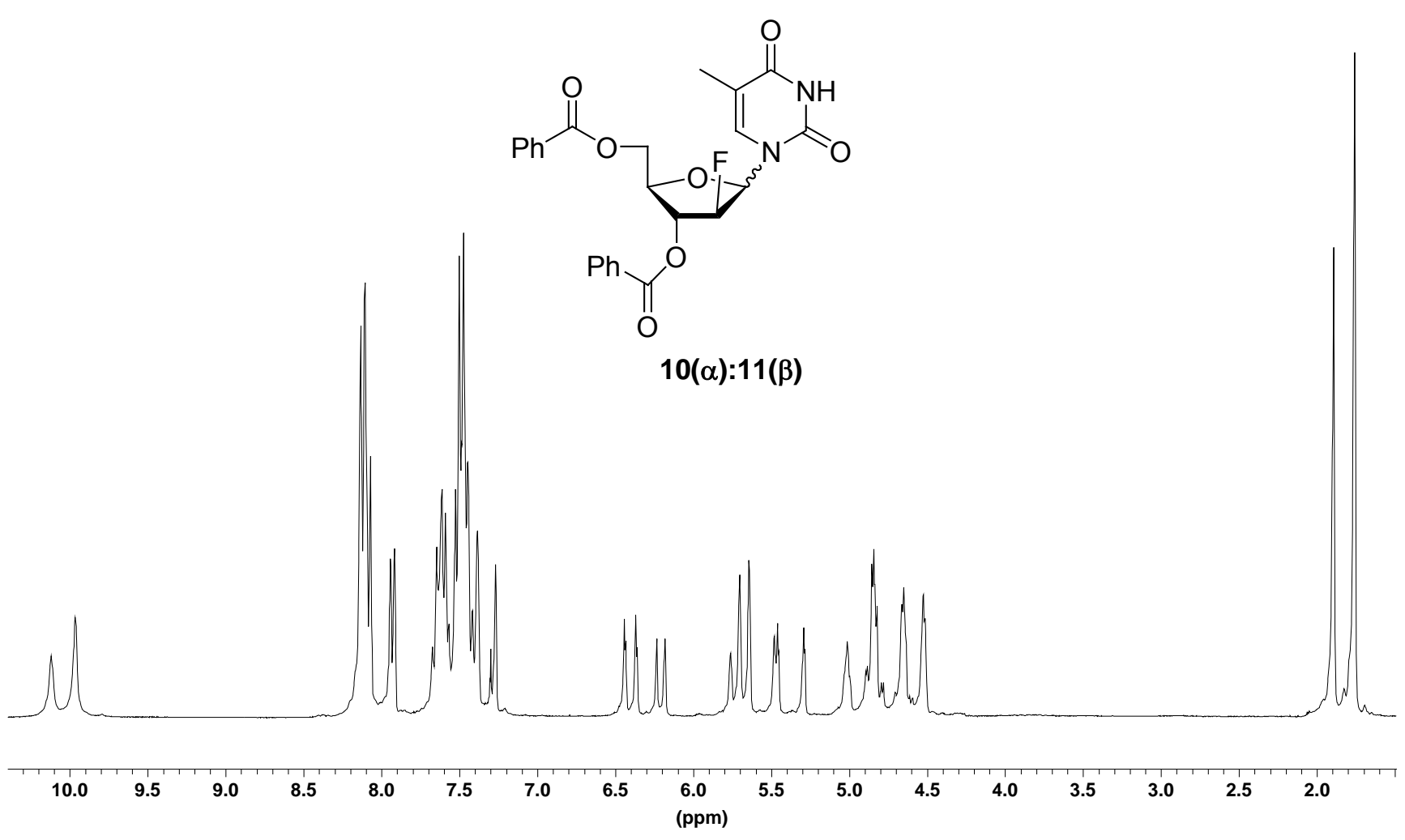


1-(3',5'-Di-O-Benzoyl-2'-Fluoro-2'-deoxy- $\alpha / \beta$-D-Arabinofuranosyl)Thymine

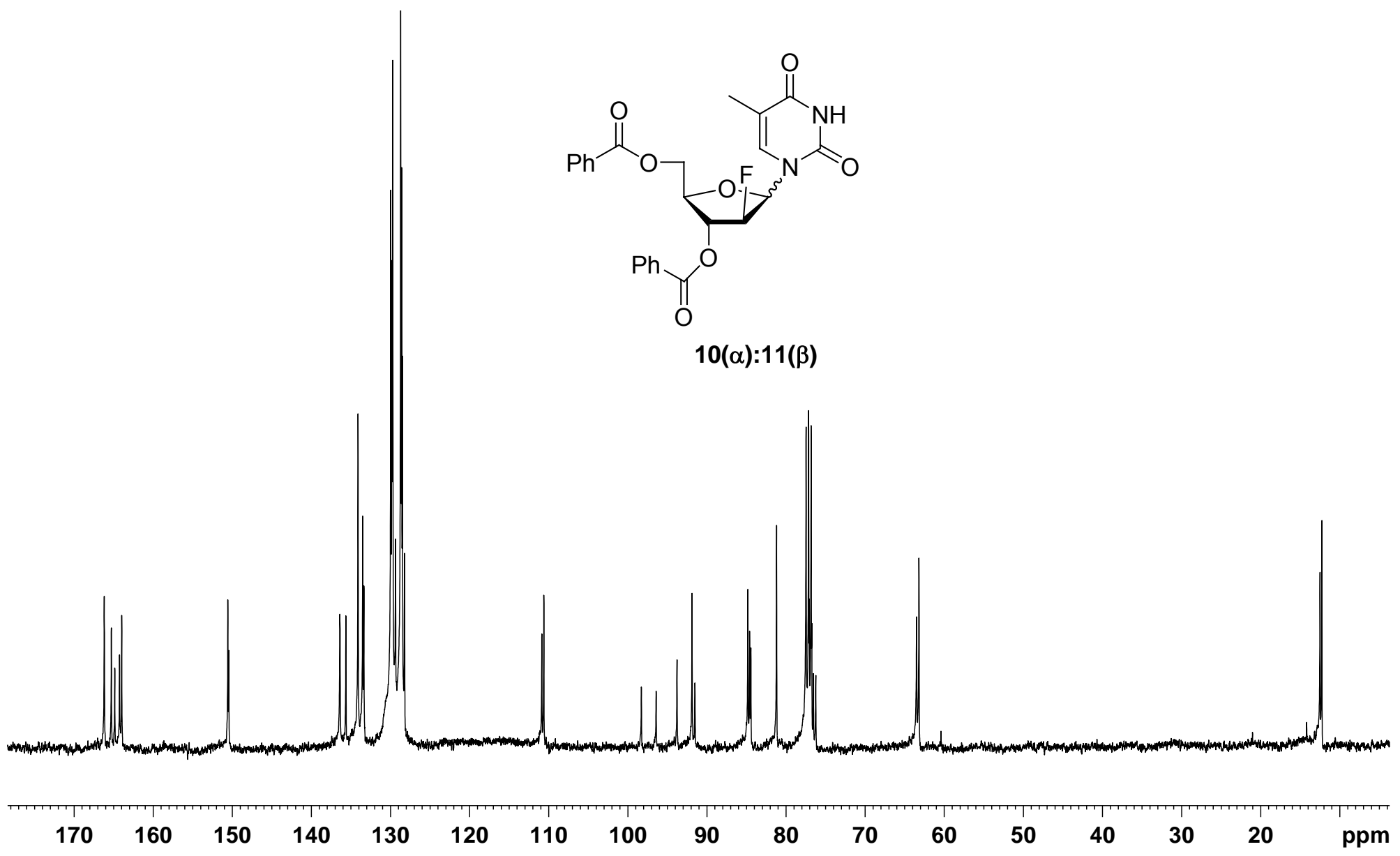




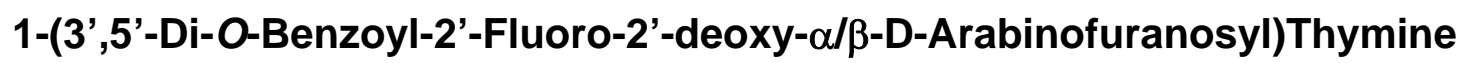

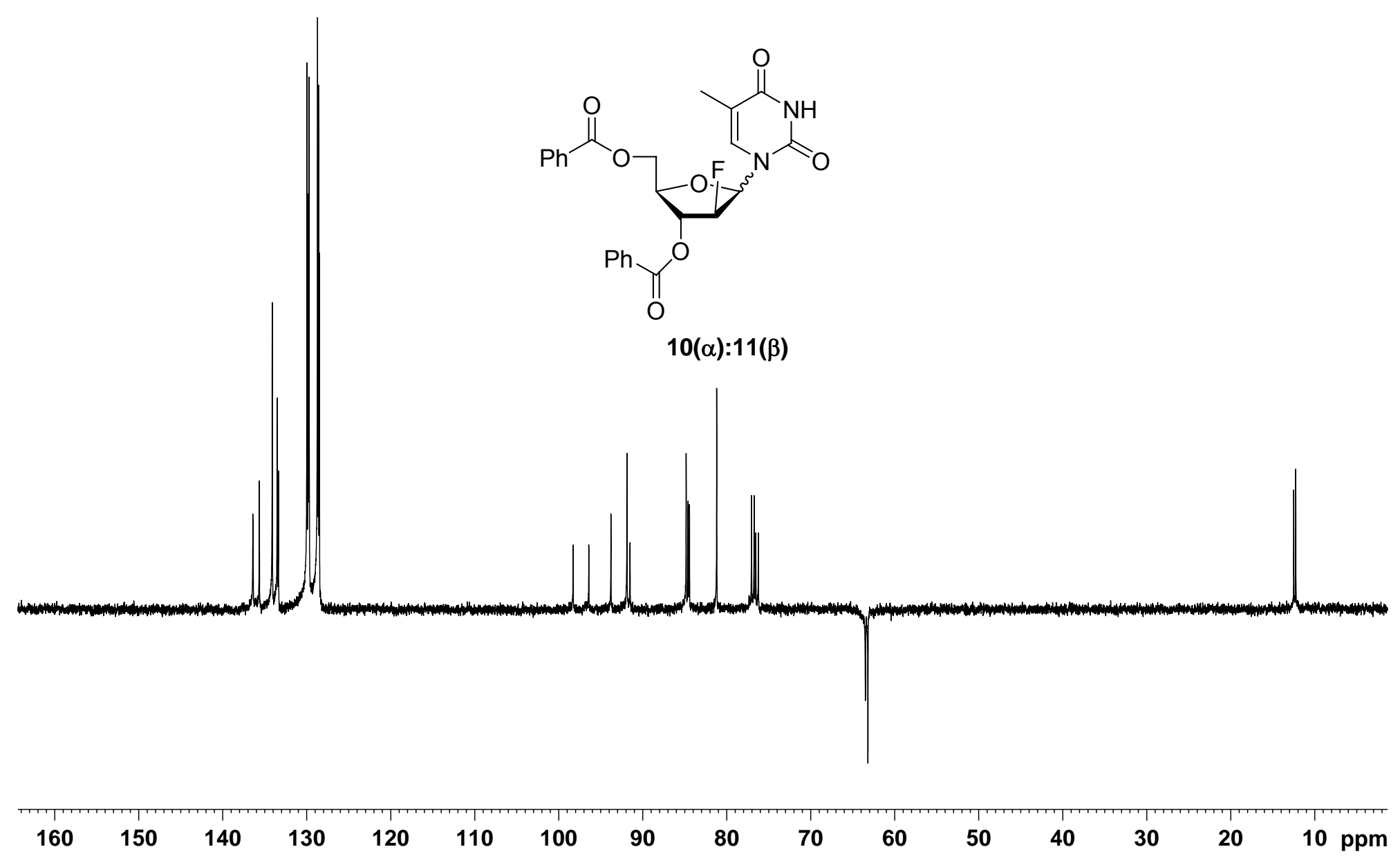




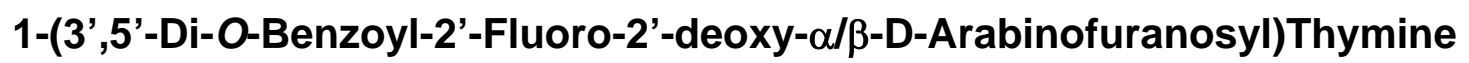

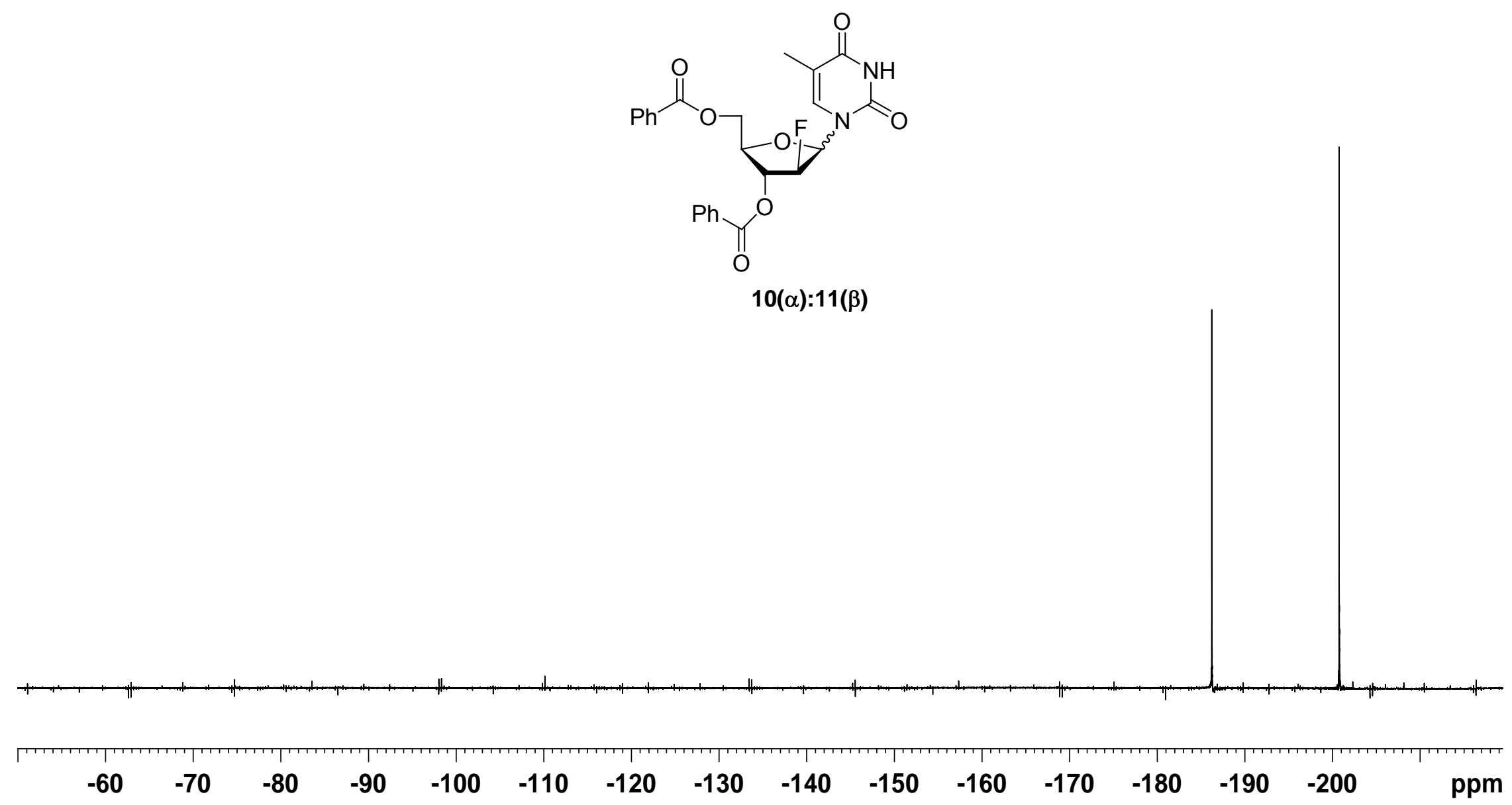




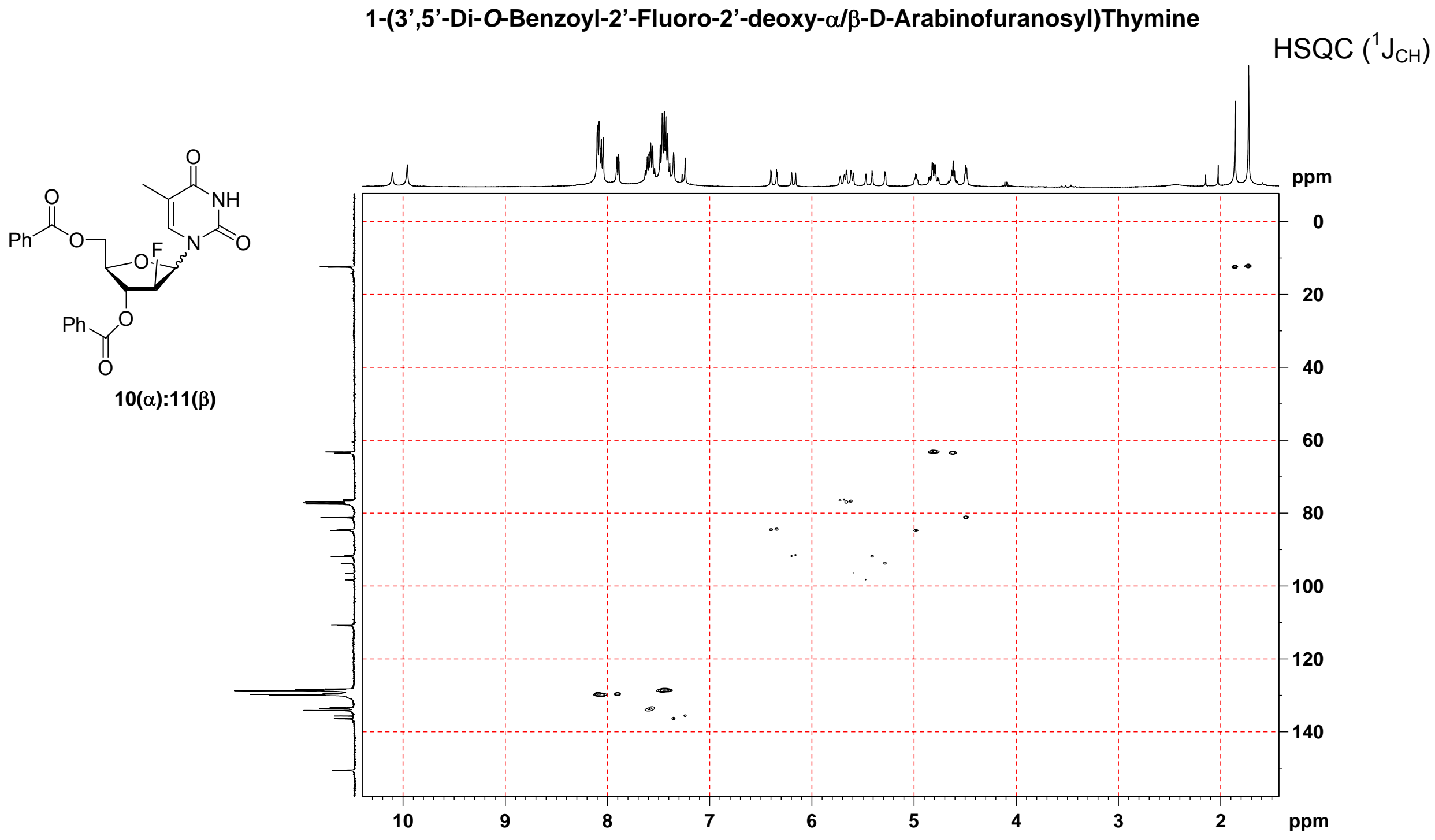




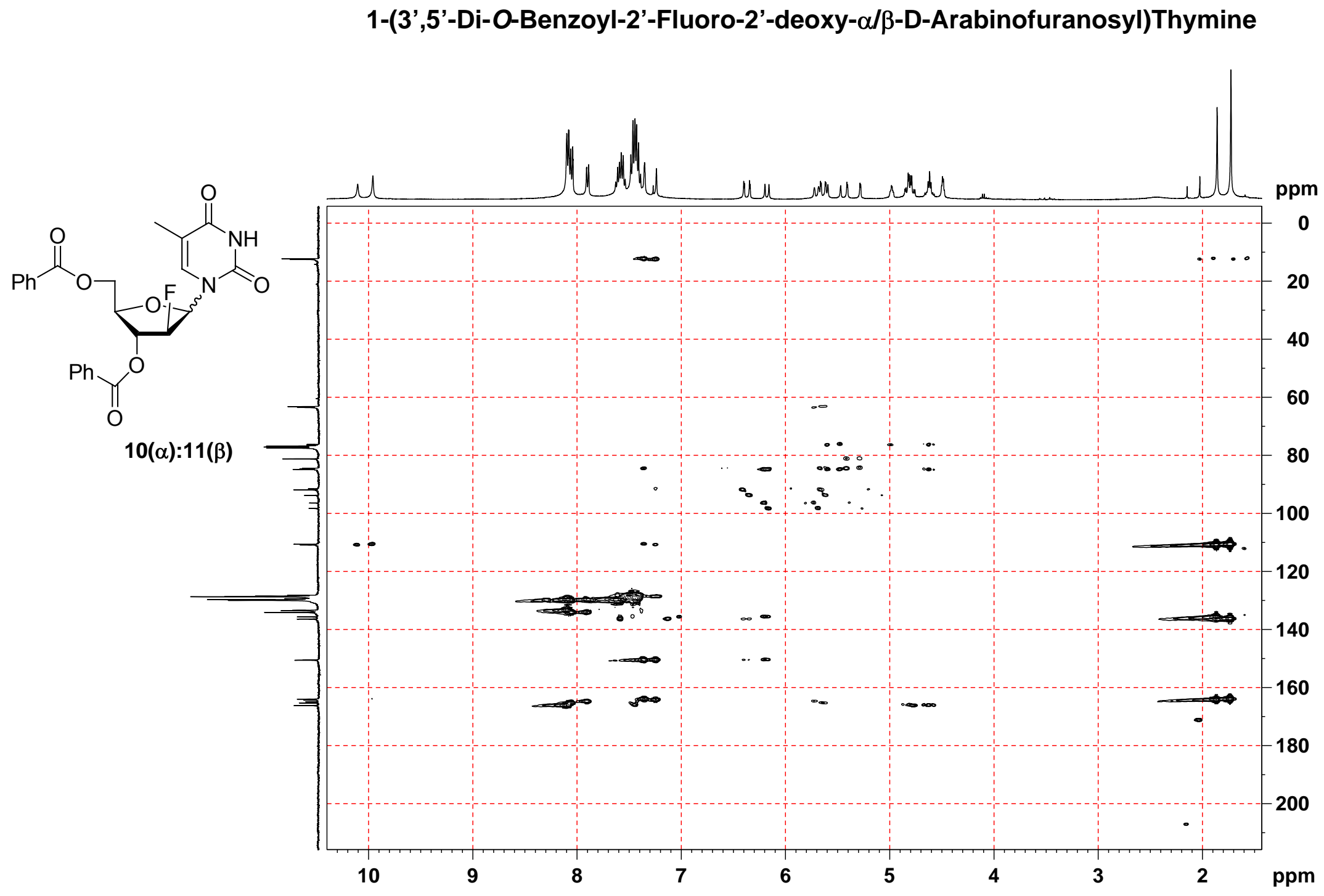




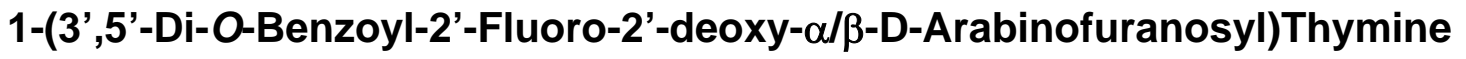
COSY

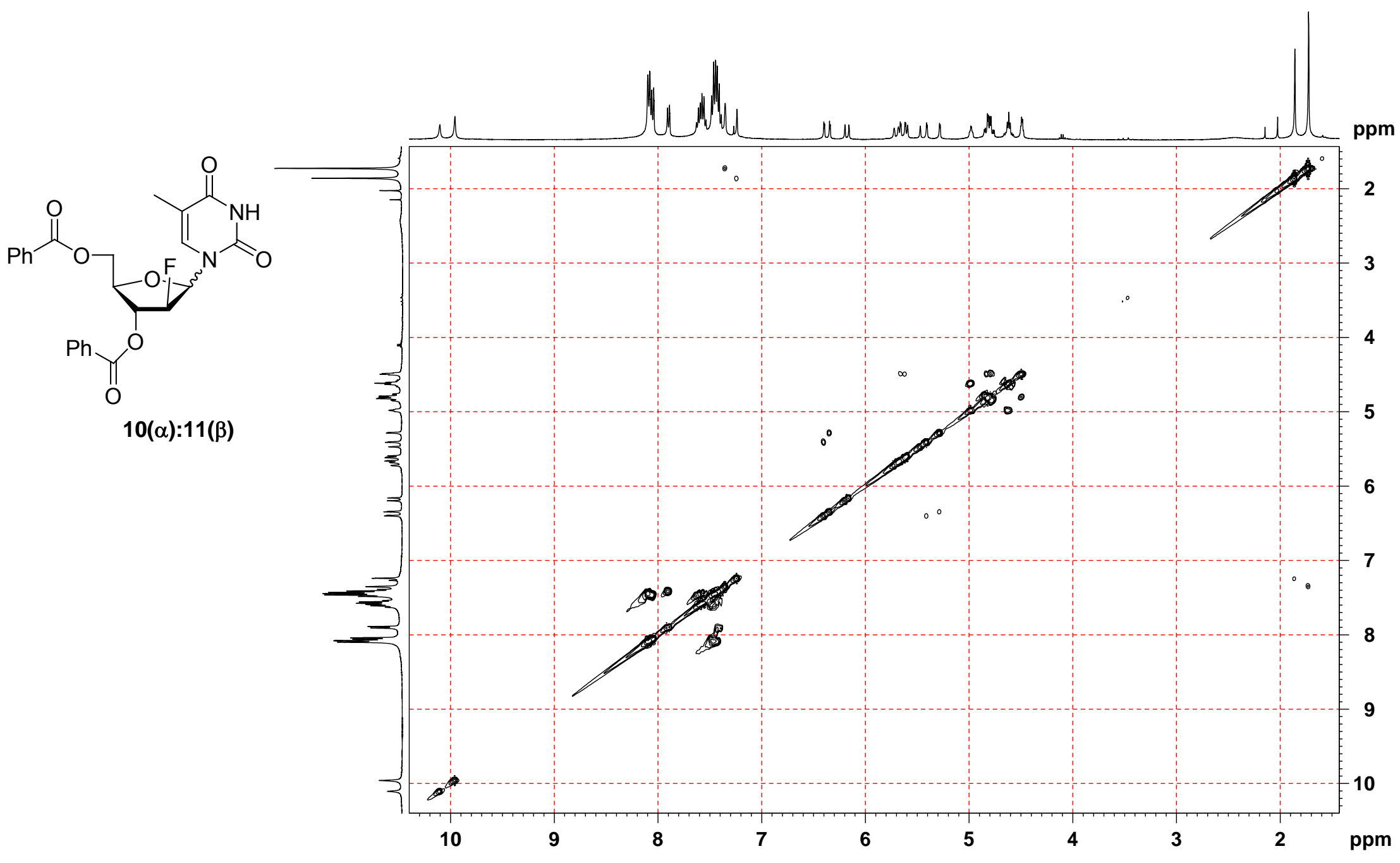




\section{1-(3'-O-Benzoyl-2'-Fluoro-2'-deoxy- $\beta$-D-Arabinofuranosyl)Thymine}

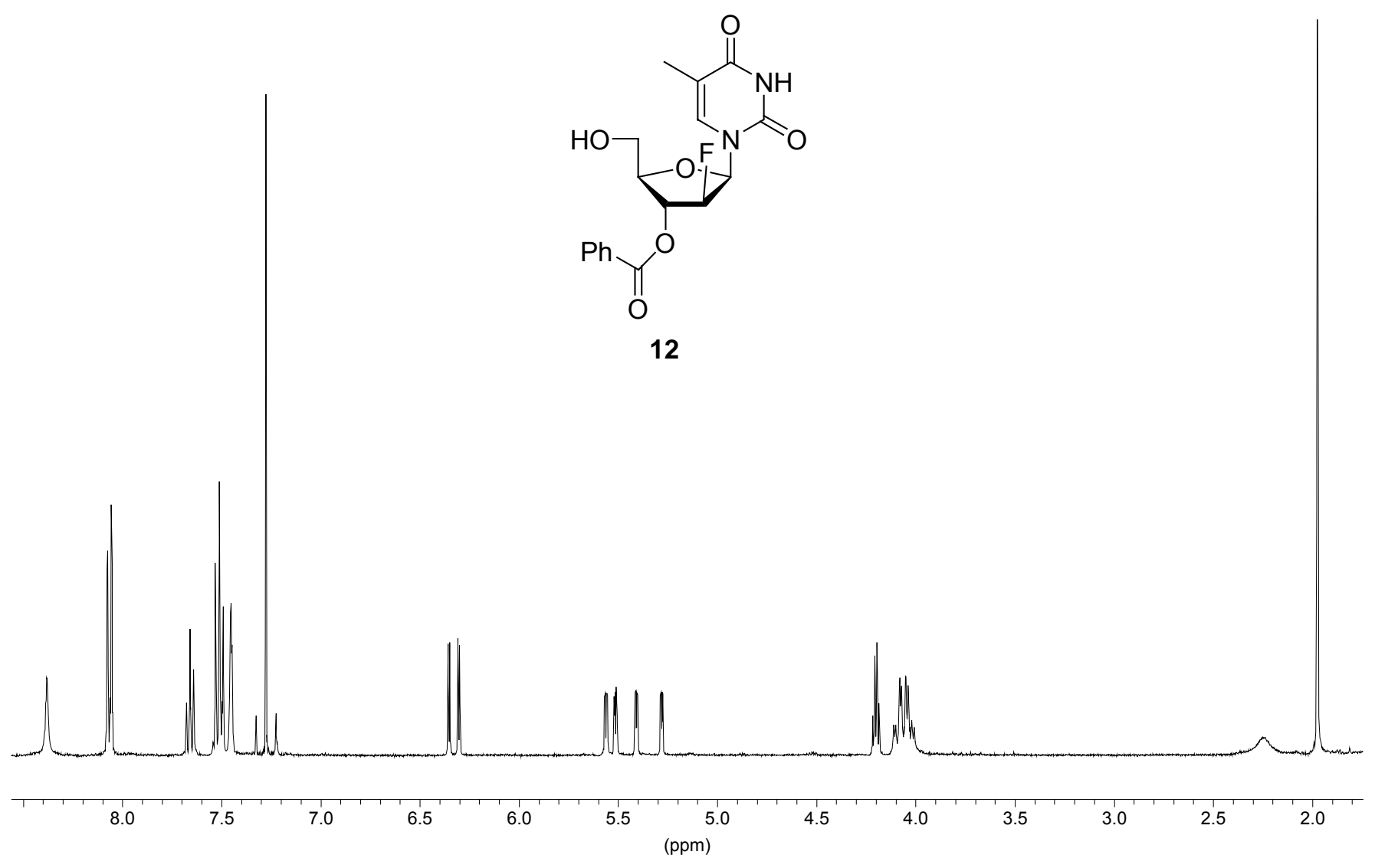




\section{1-(3'-O-Benzoyl-2'-Fluoro-2'-deoxy- $\beta$-D-Arabinofuranosyl)Thymine}

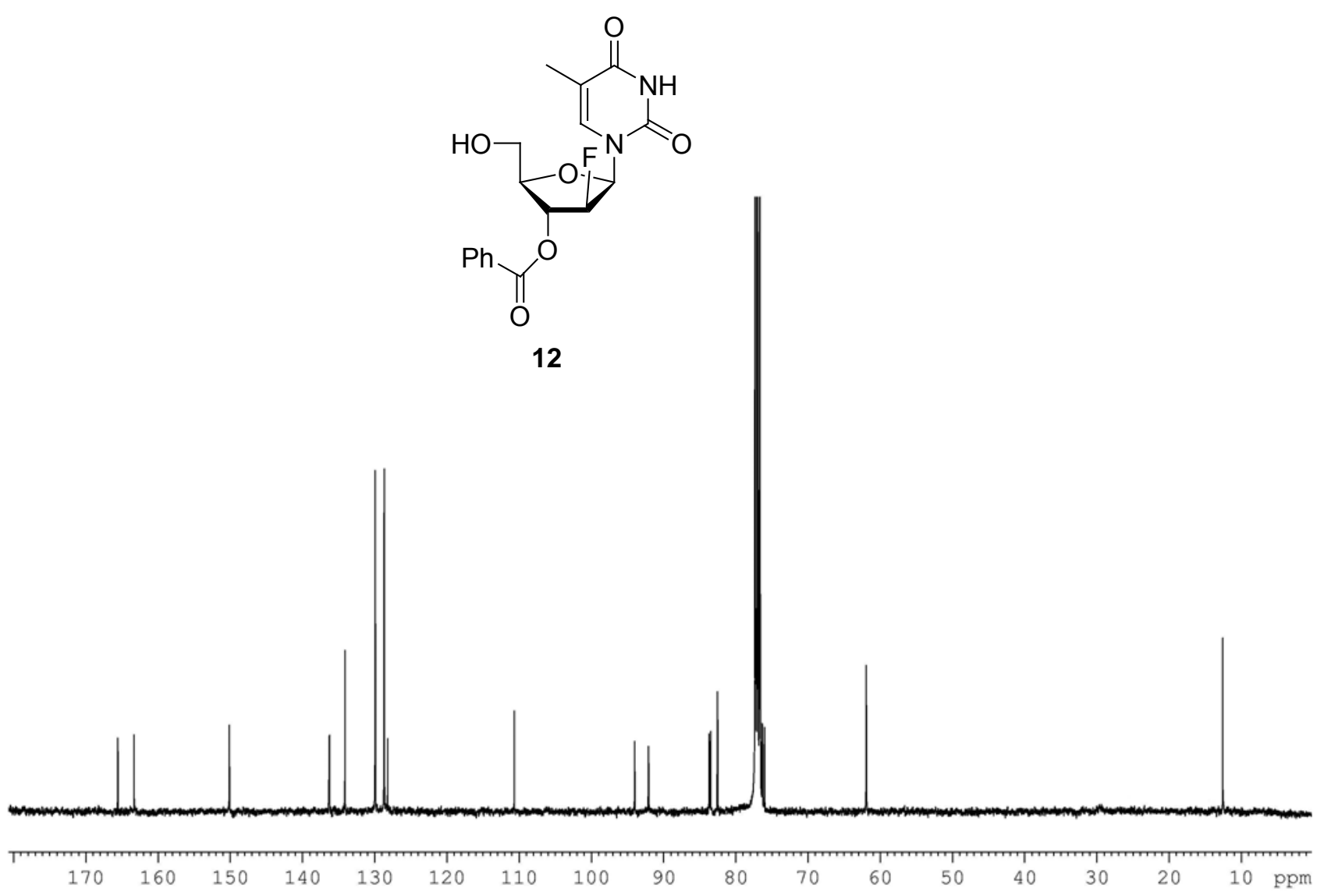




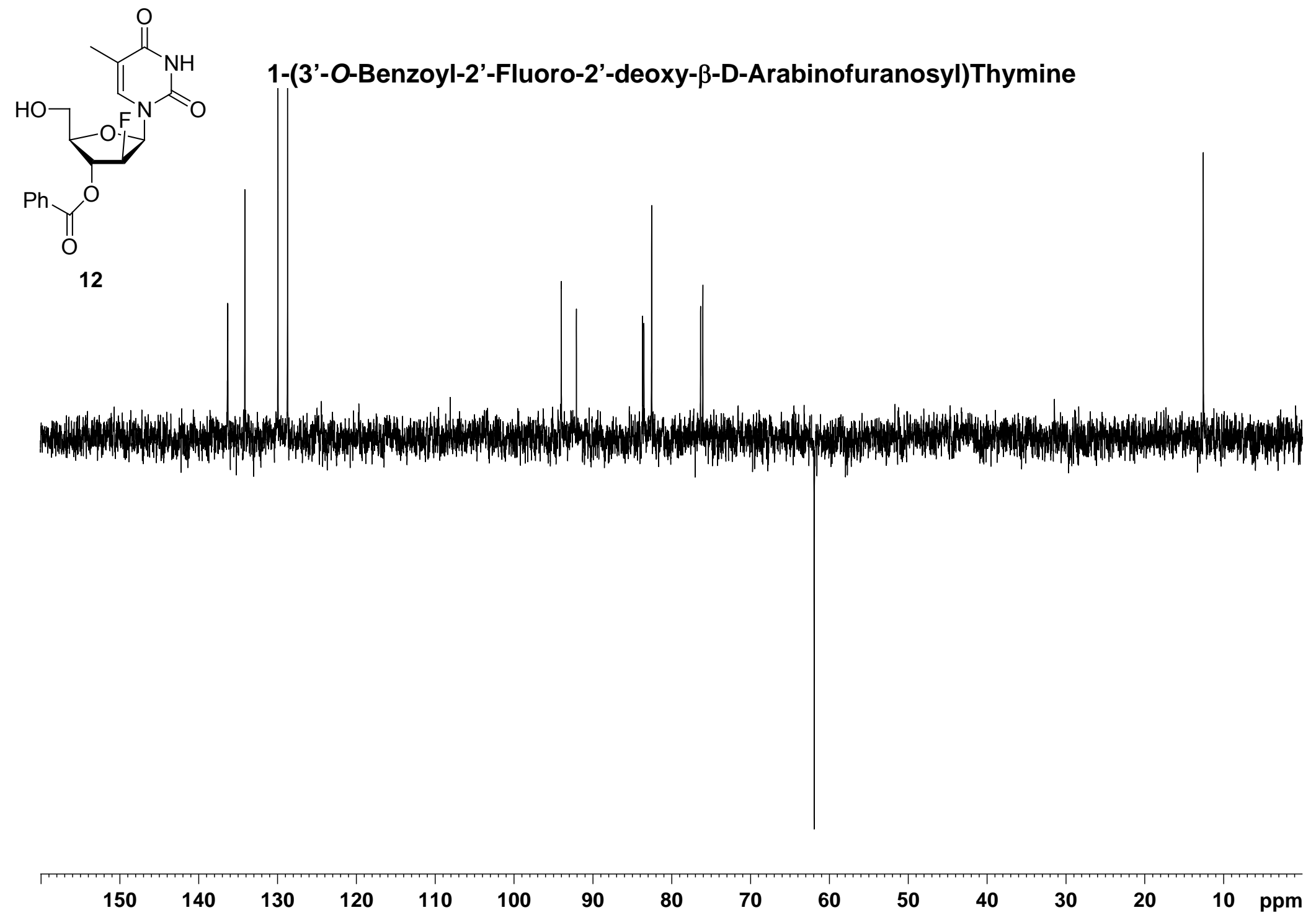




\section{1-(3'-O-Benzoyl-2'-Fluoro-2'-deoxy- $\beta$-D-Arabinofuranosyl)Thymine}
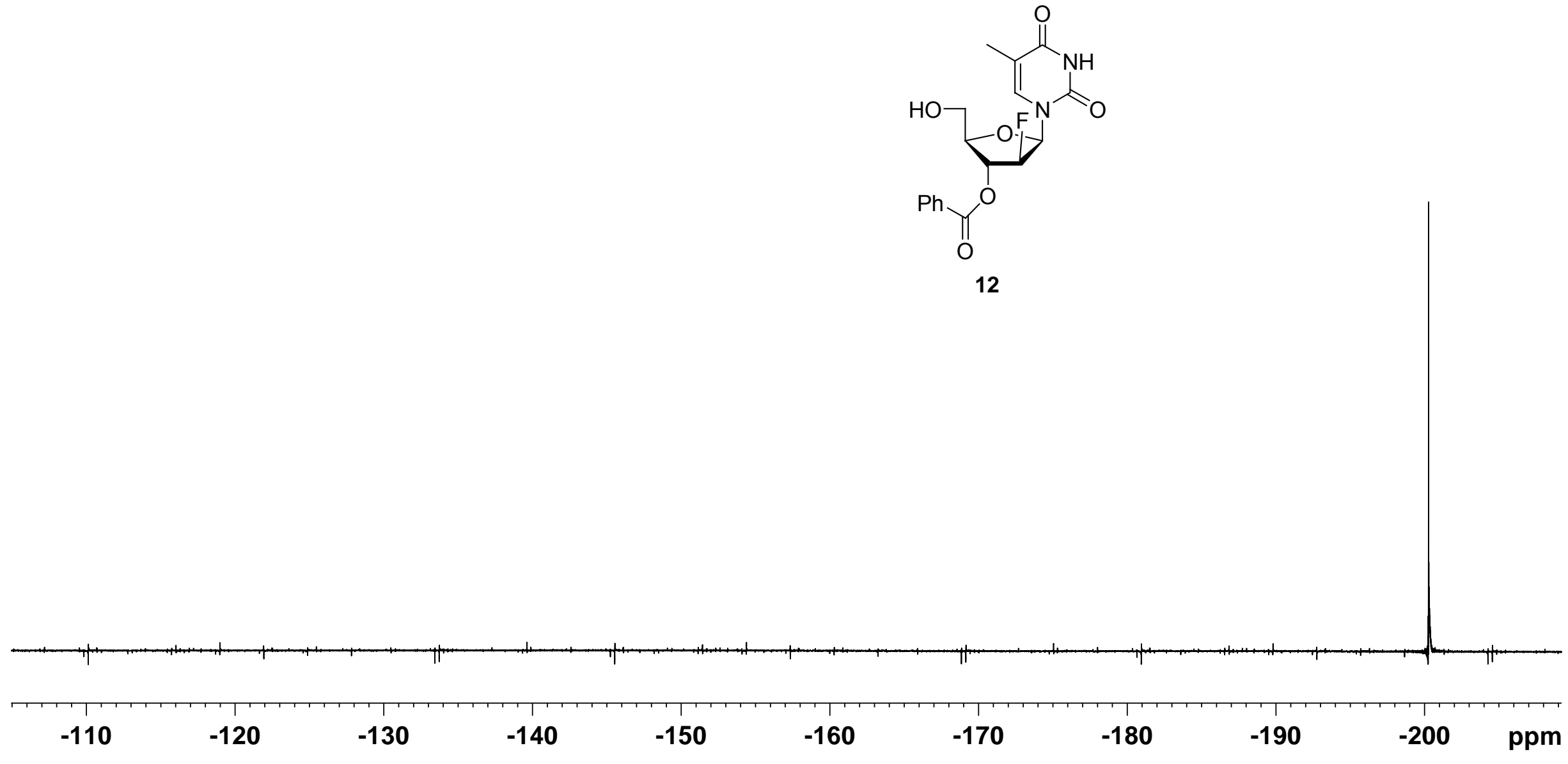


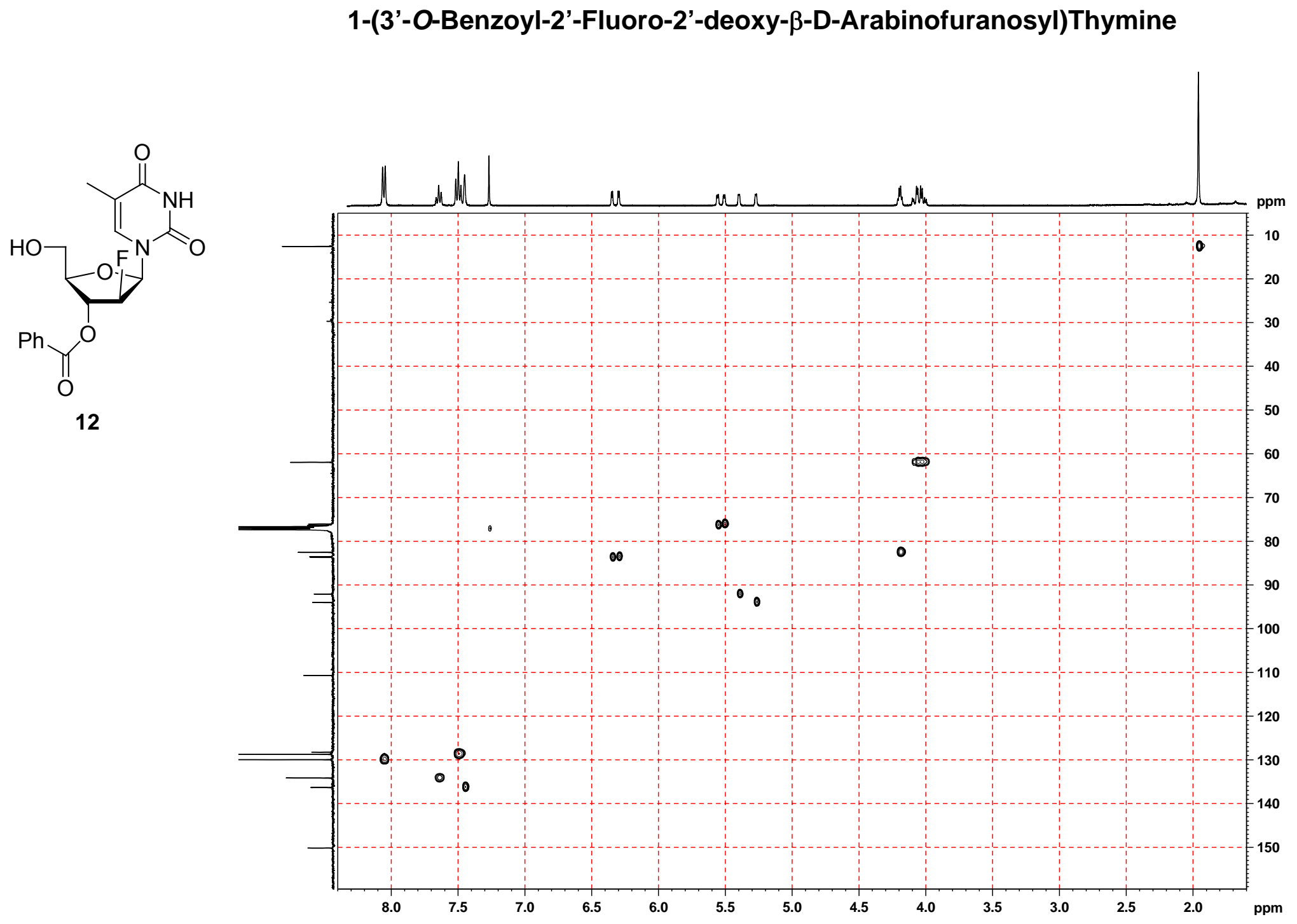

HSQC $\left({ }^{1} \mathrm{~J}_{\mathrm{CH}}\right)$ 


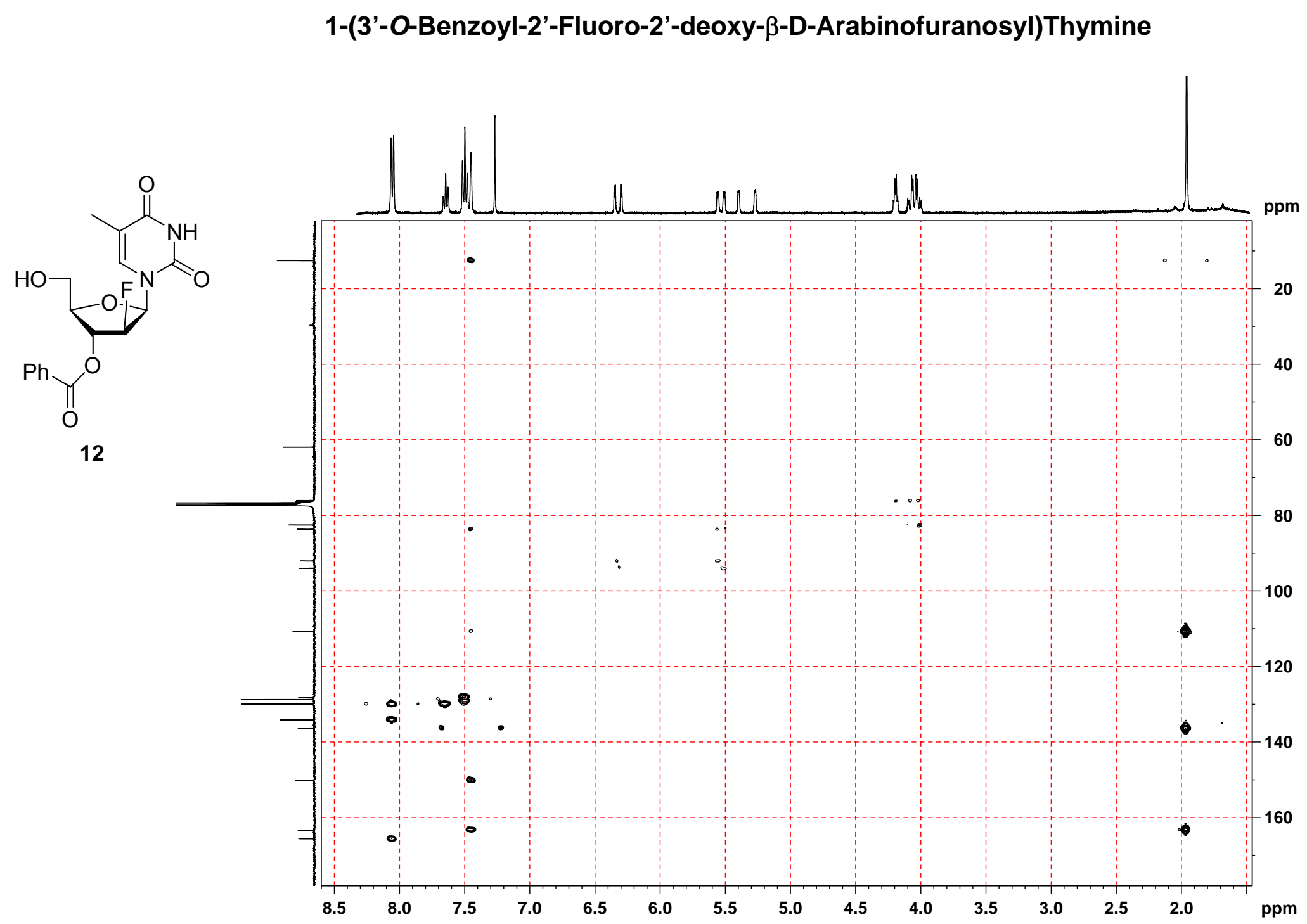

HMBC $\left({ }^{3} \mathrm{~J}_{\mathrm{CH}}\right)$ 


\section{1-(3'-O-Benzoyl-2'-Fluoro-2'-deoxy- $\beta$-D-Arabinofuranosyl)Thymine}

\section{COSY}
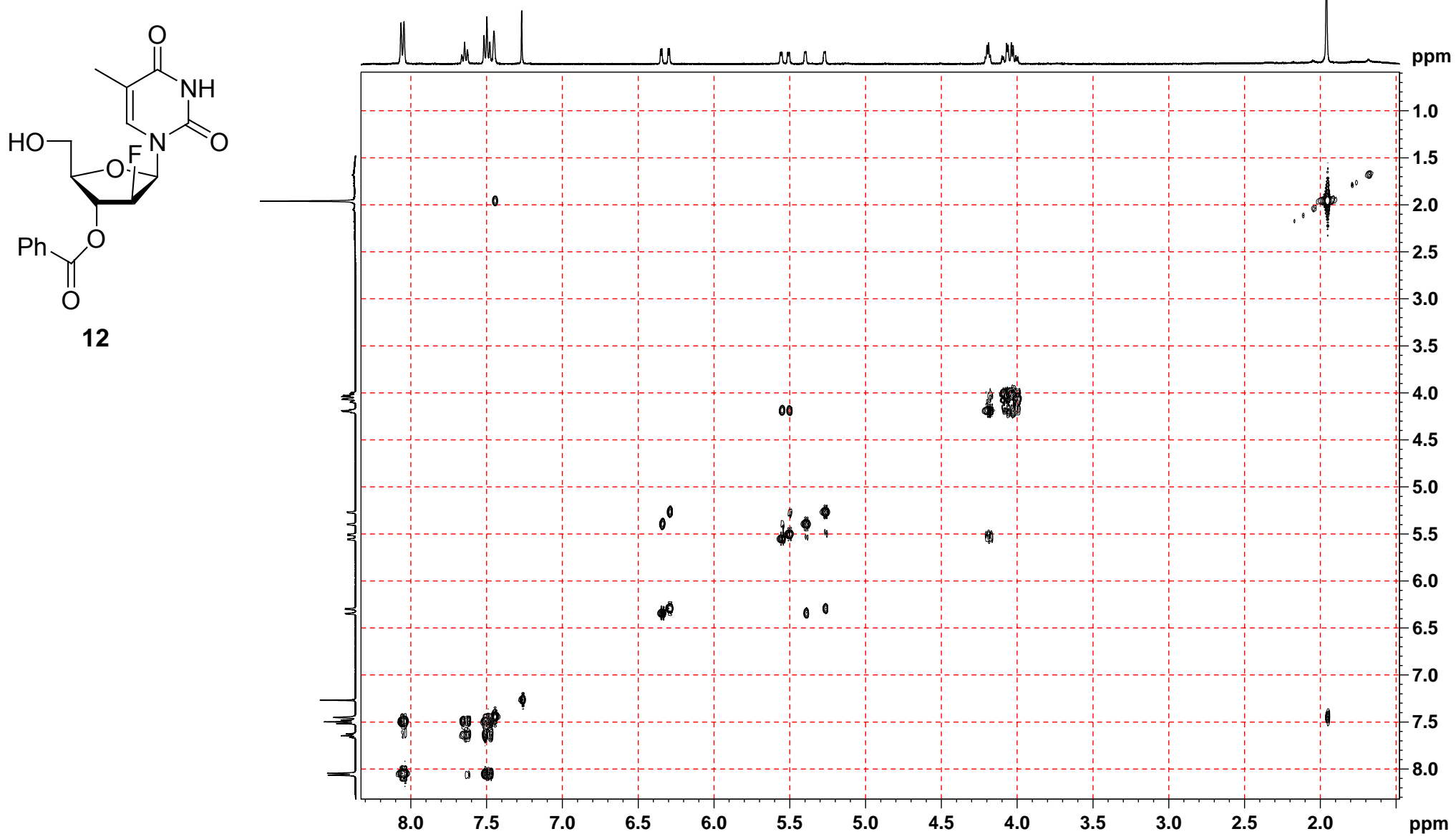


\section{1-(3'-O-Benzoyl-2'-Fluoro-2'-deoxy- $\beta$-D-Arabinofuranosyl)Thymine}

\section{NOESY}
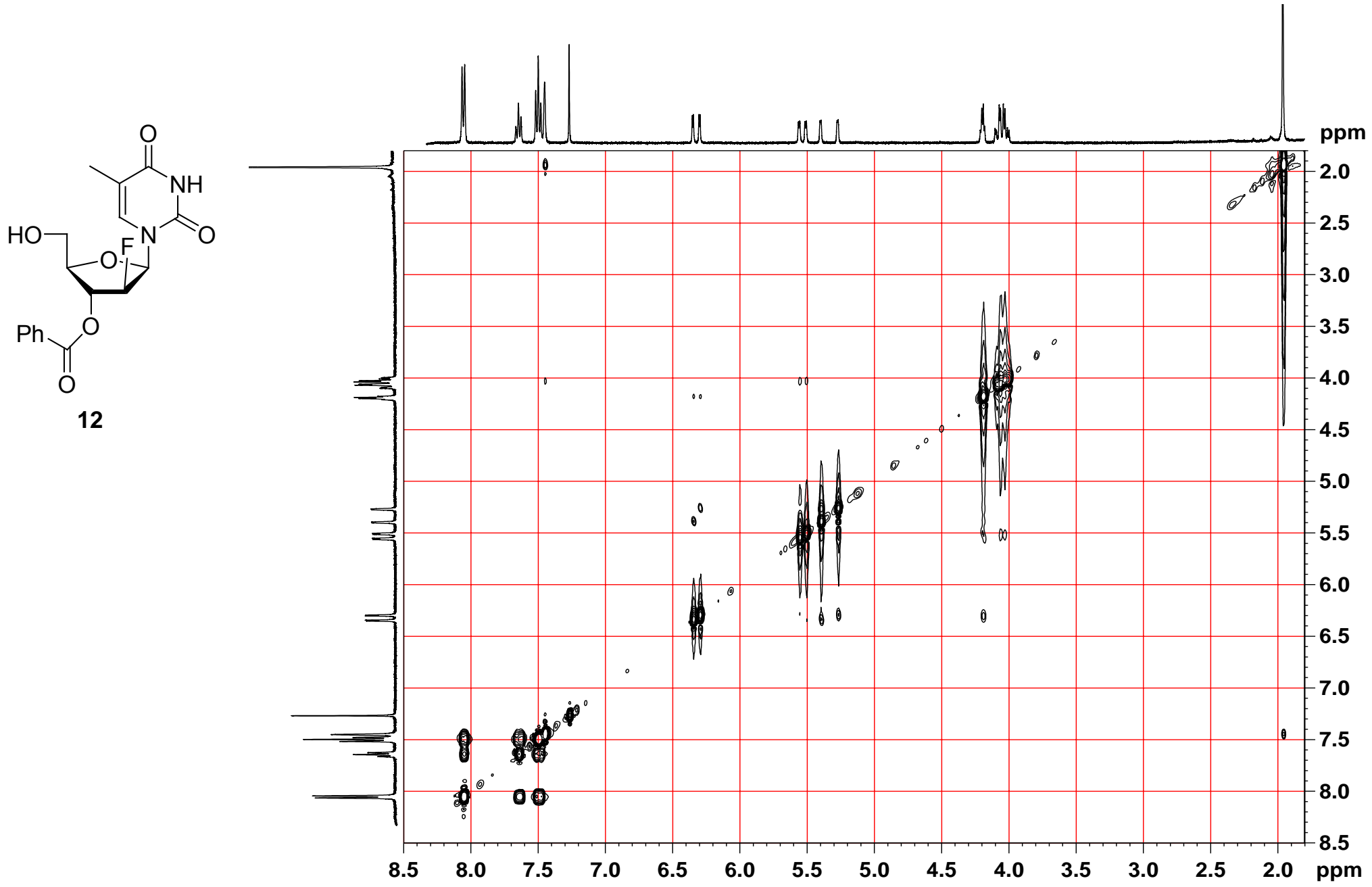


\section{1-(3'-O-Benzoyl-2'-Fluoro-2'-deoxy- $\alpha$-D-Arabinofuranosyl)Thymine}

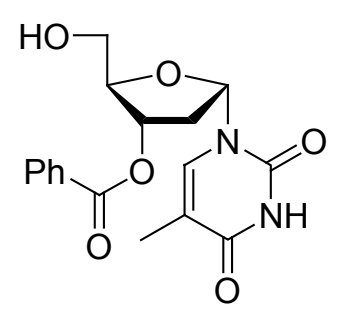

13

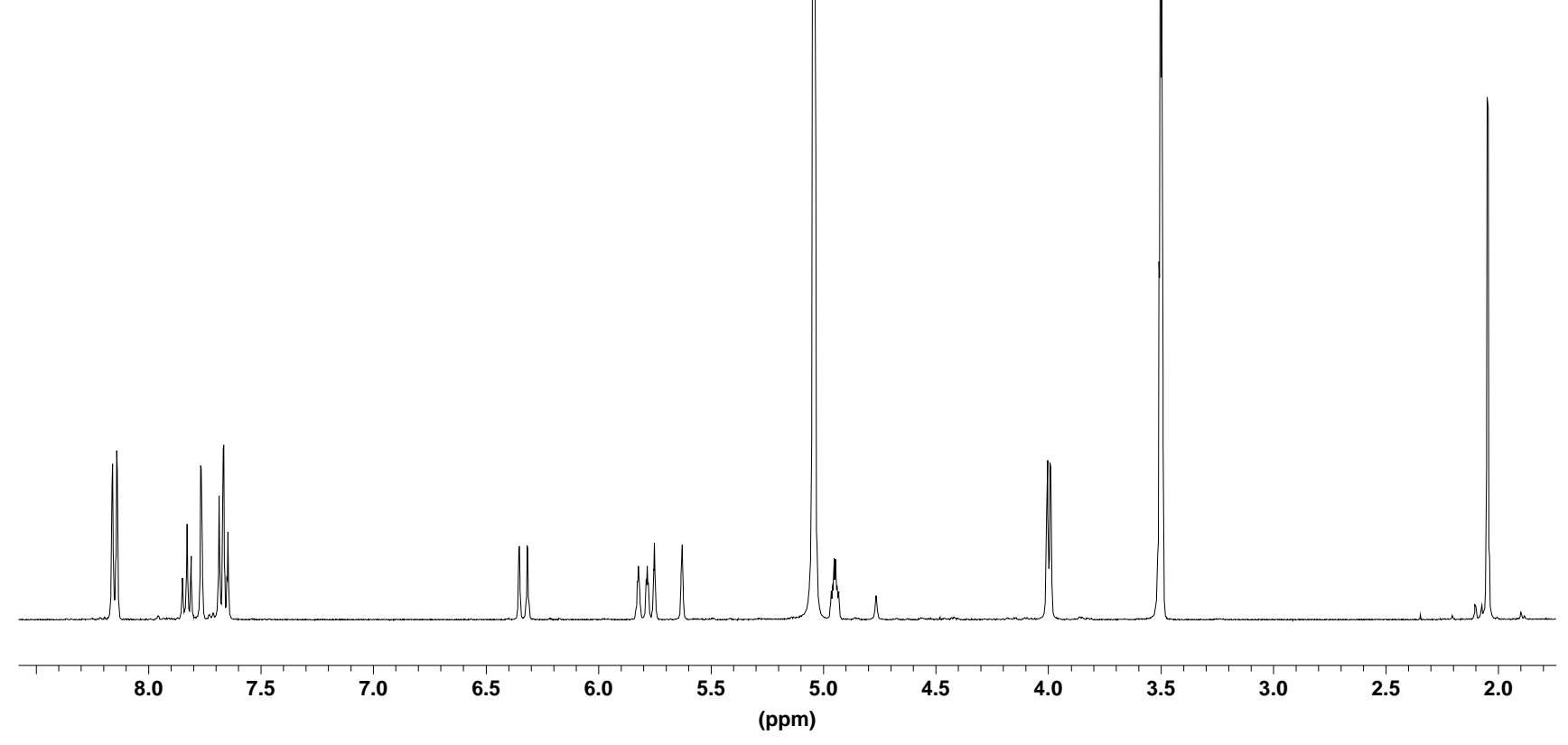




\section{1-(3'-O-Benzoyl-2'-Fluoro-2'-deoxy- $\alpha$-D-Arabinofuranosyl)Thymine}

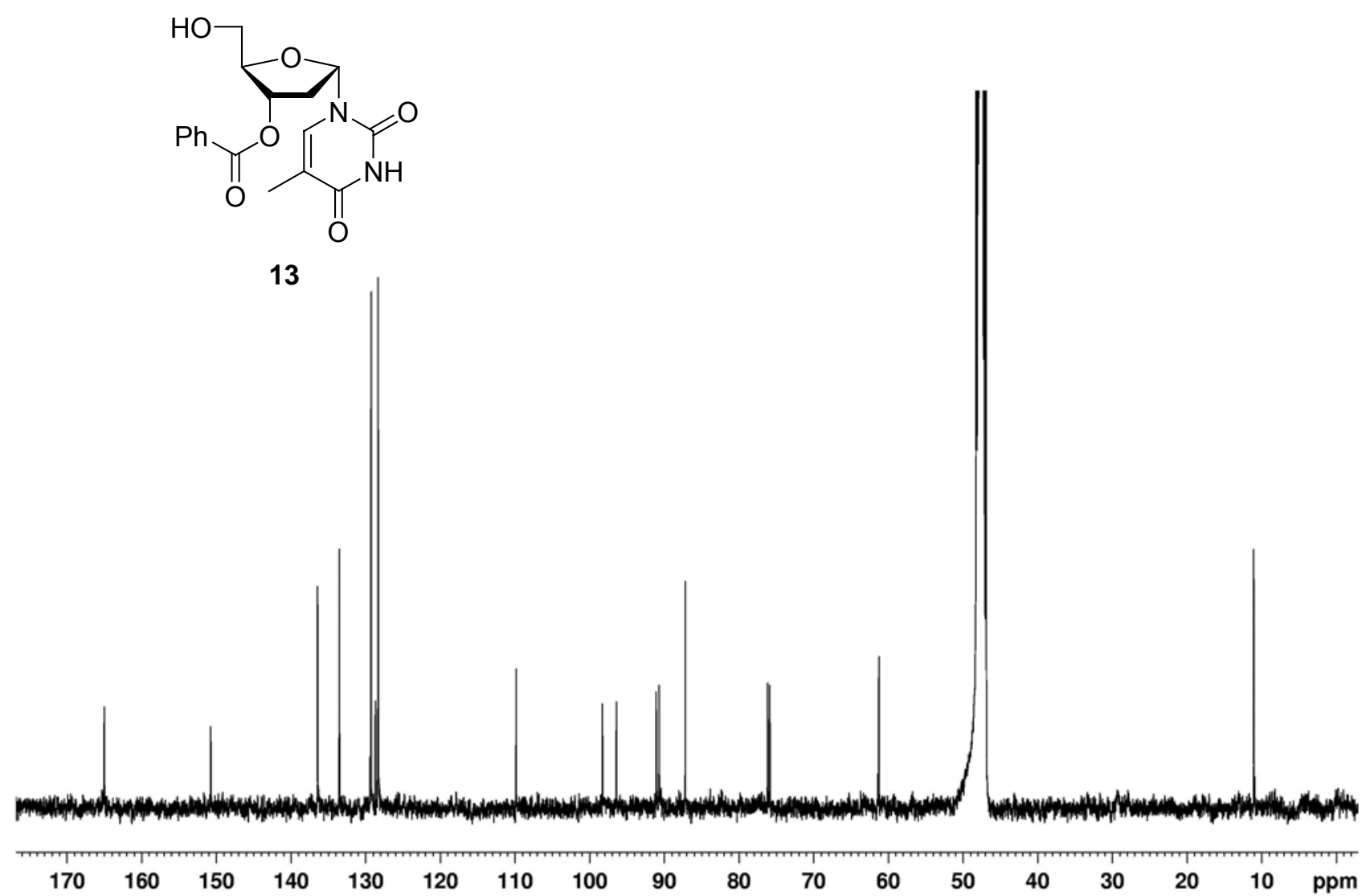


1-(3'-O-Benzoyl-2'-Fluoro-2'-deoxy- $\alpha$-D-Arabinofuranosyl)Thymine

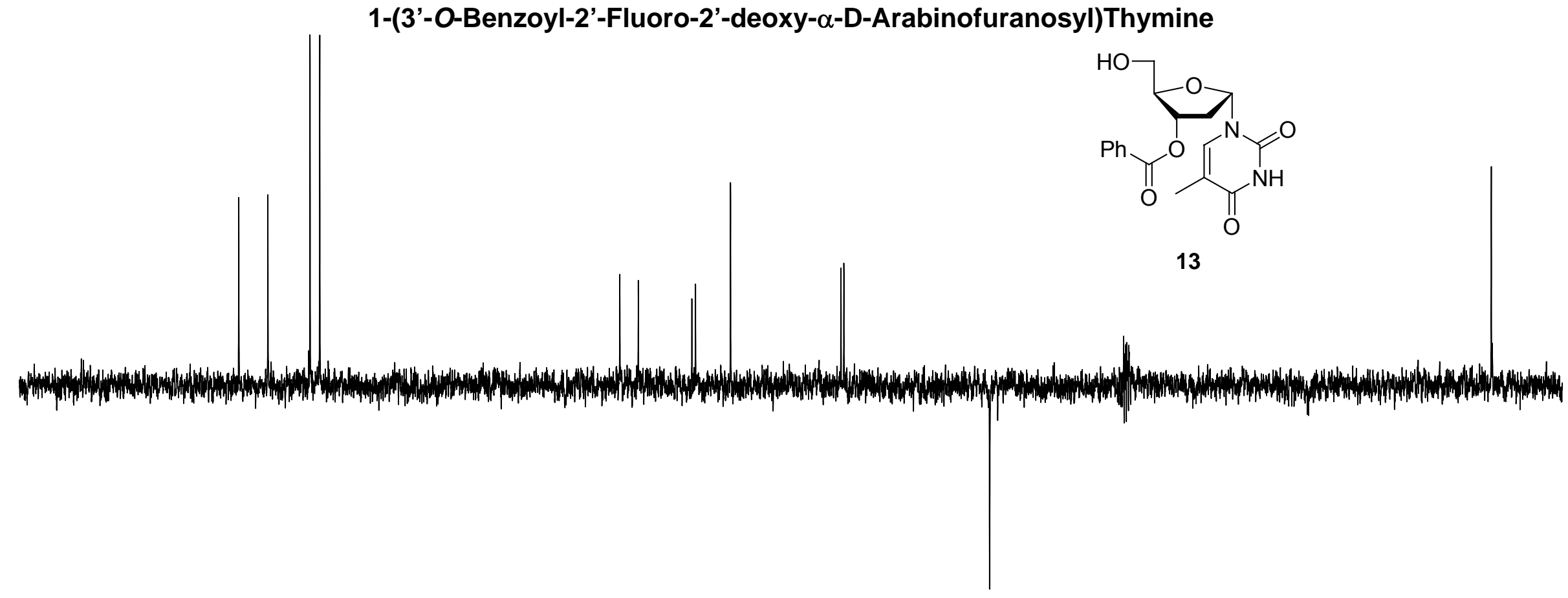

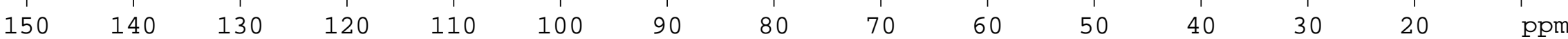


1-(3'-O-Benzoyl-2'-Fluoro-2'-deoxy- $\alpha$-D-Arabinofuranosyl)Thymine

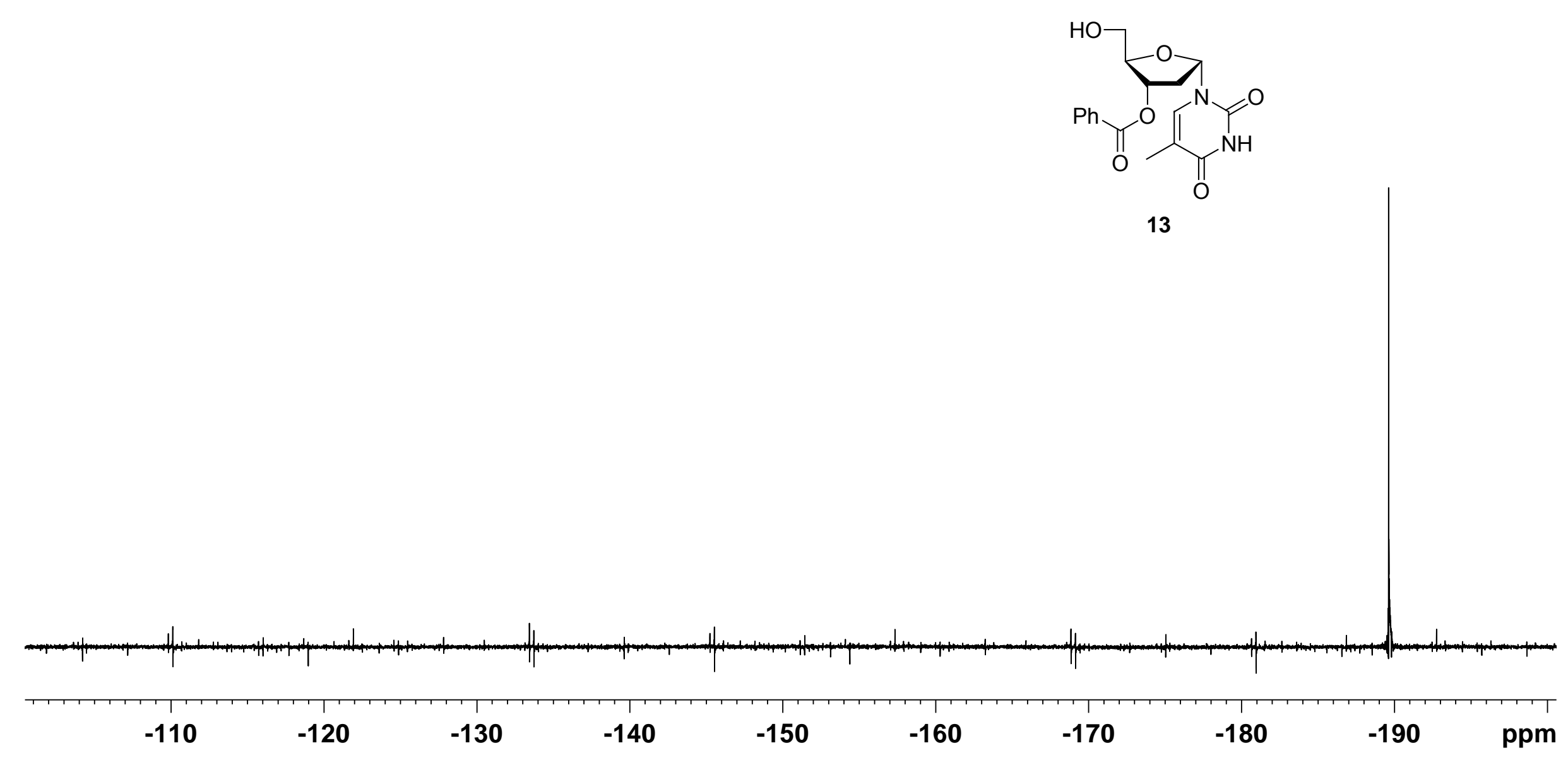




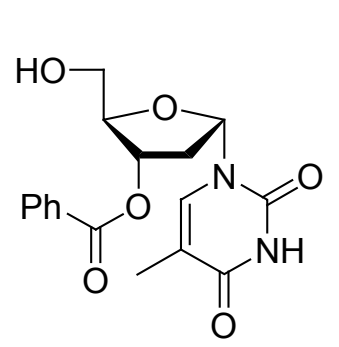

1-(3'-O-Benzoyl-2'-Fluoro-2'-deoxy- $\alpha$-D-Arabinofuranosyl)Thymine

13



$\operatorname{HSQC}\left({ }^{1} \mathrm{~J}_{\mathrm{CH}}\right)$ 


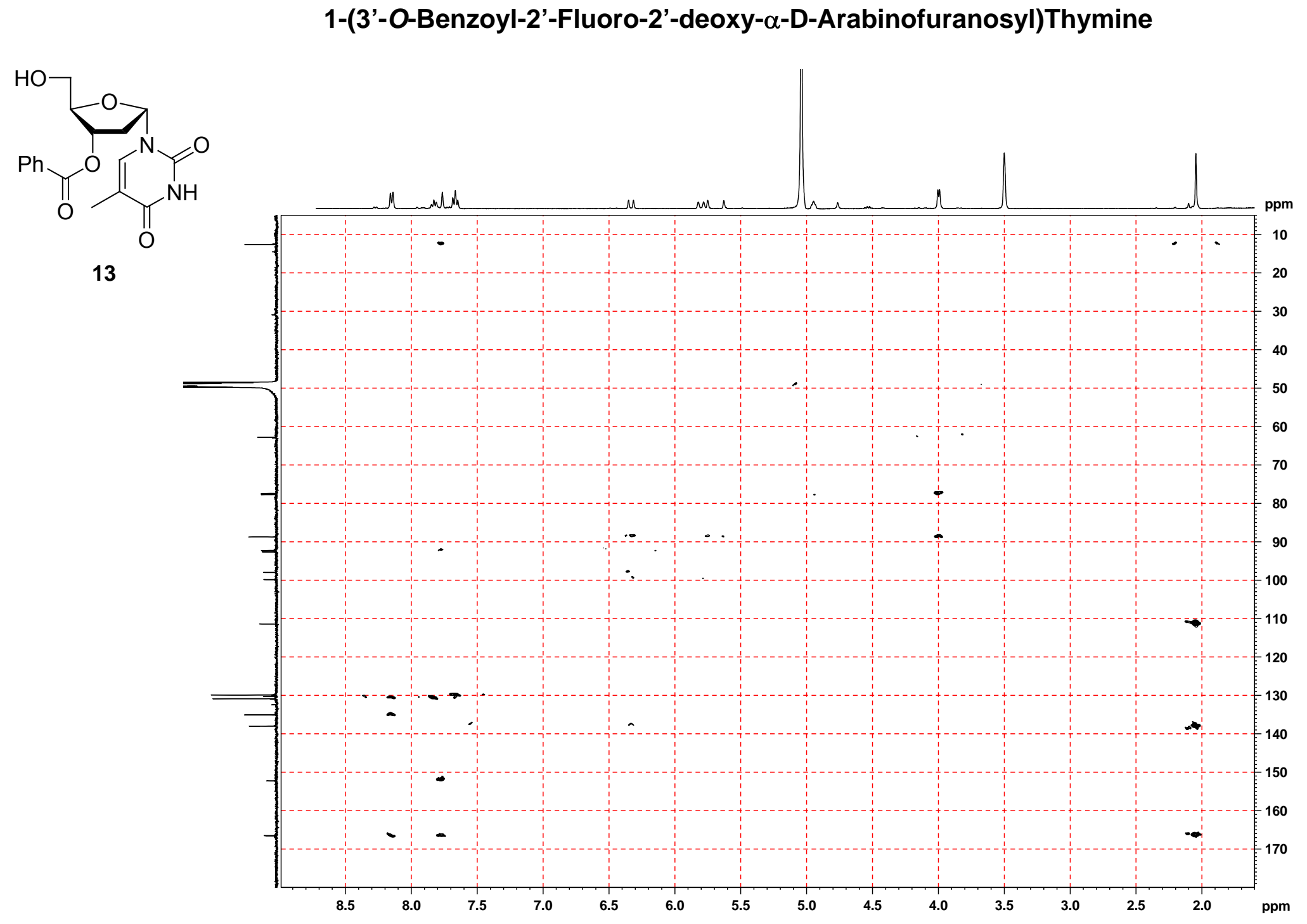

HMBC $\left({ }^{3} \mathrm{~J}_{\mathrm{CH}}\right)$ 
1-(3'-O-Benzoyl-2'-Fluoro-2'-deoxy- $\alpha$-D-Arabinofuranosyl)Thymine

cosy

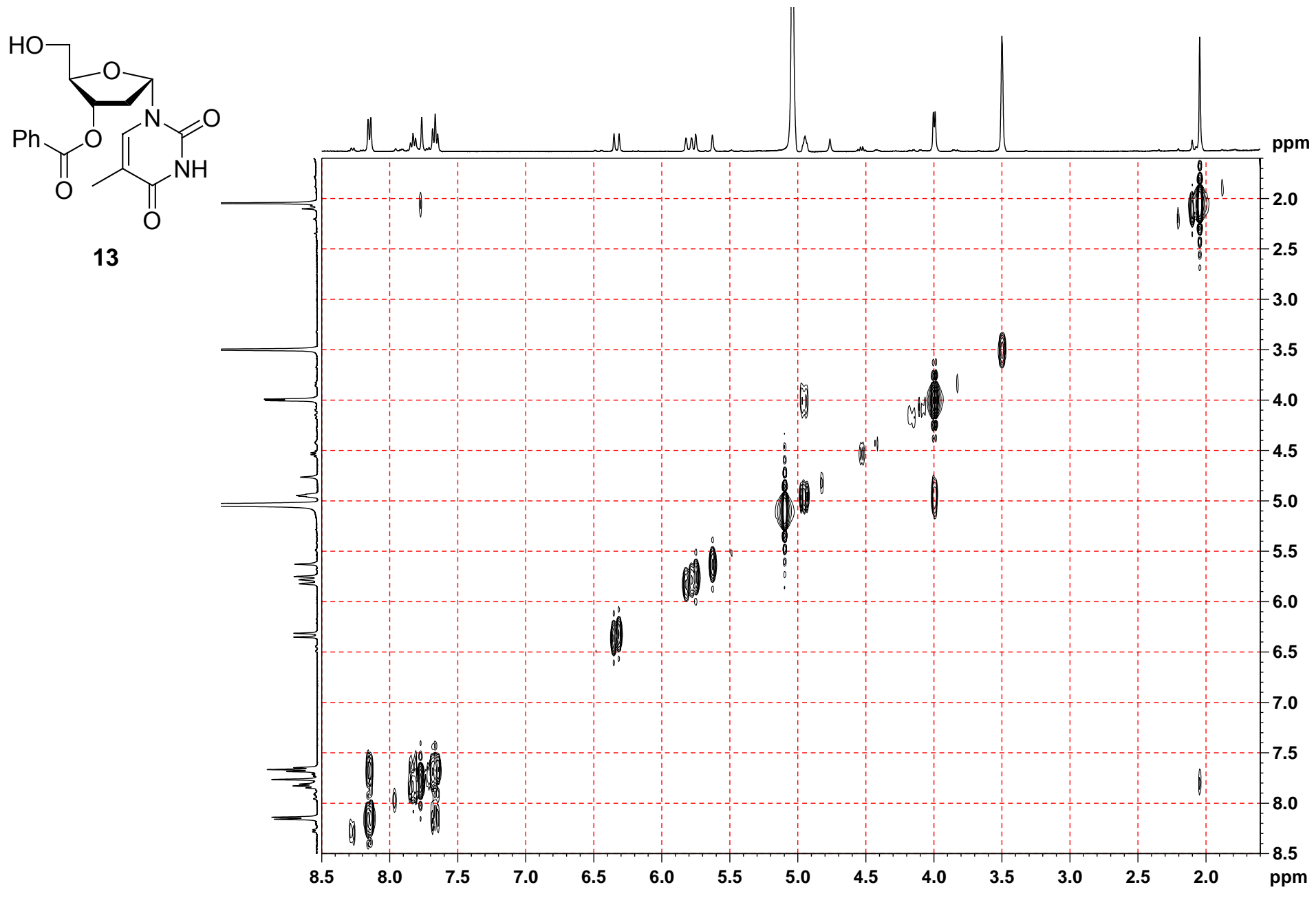


1-(3'-O-Benzoyl-2'-Fluoro-2'-deoxy- $\alpha$-D-Arabinofuranosyl)Thymine

\section{NOESY}

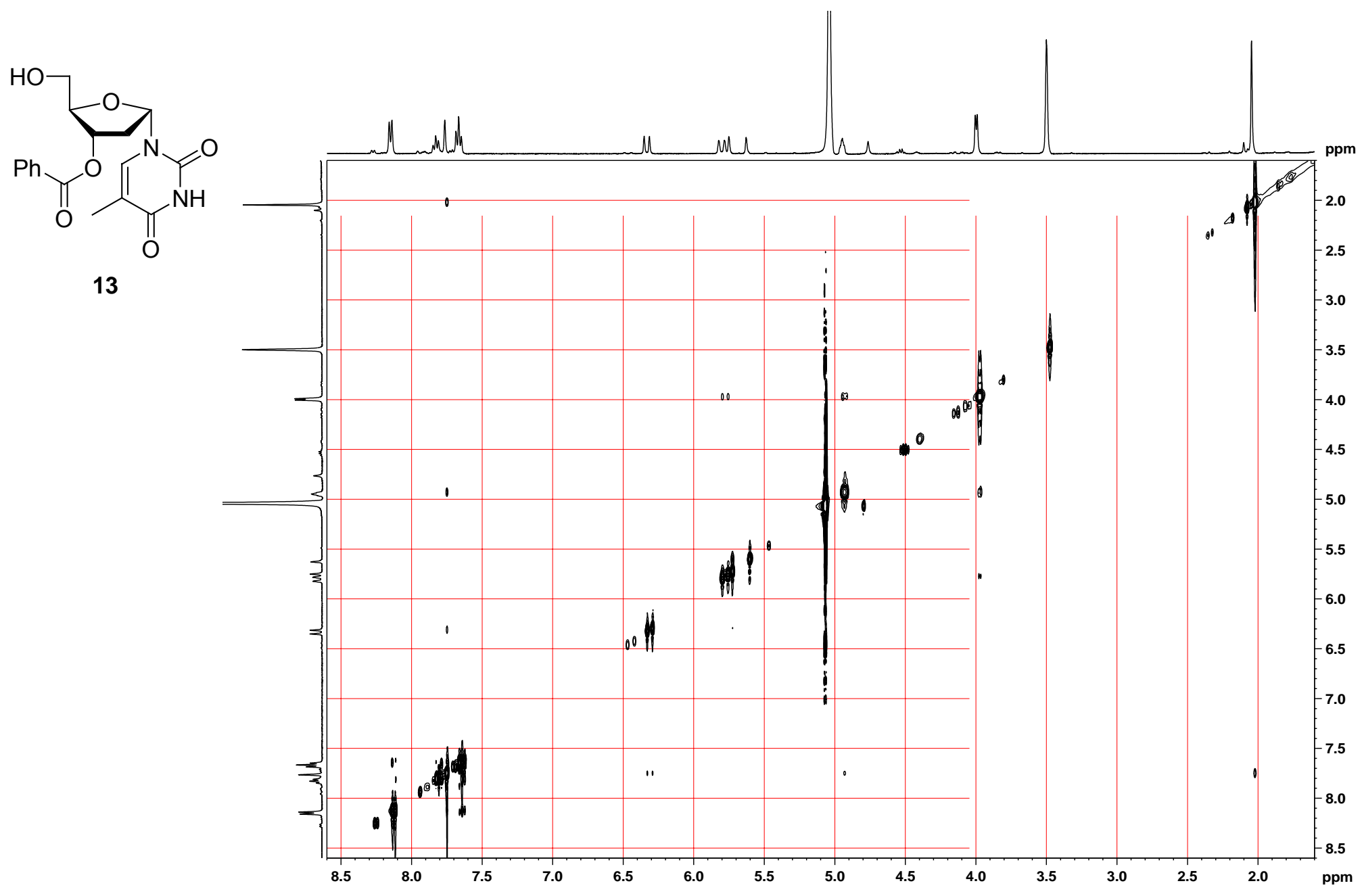




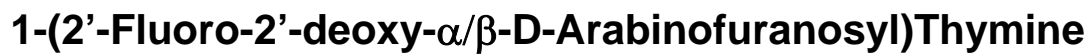

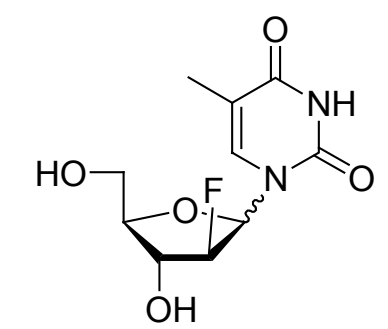

14( $\alpha): 15(\beta)$

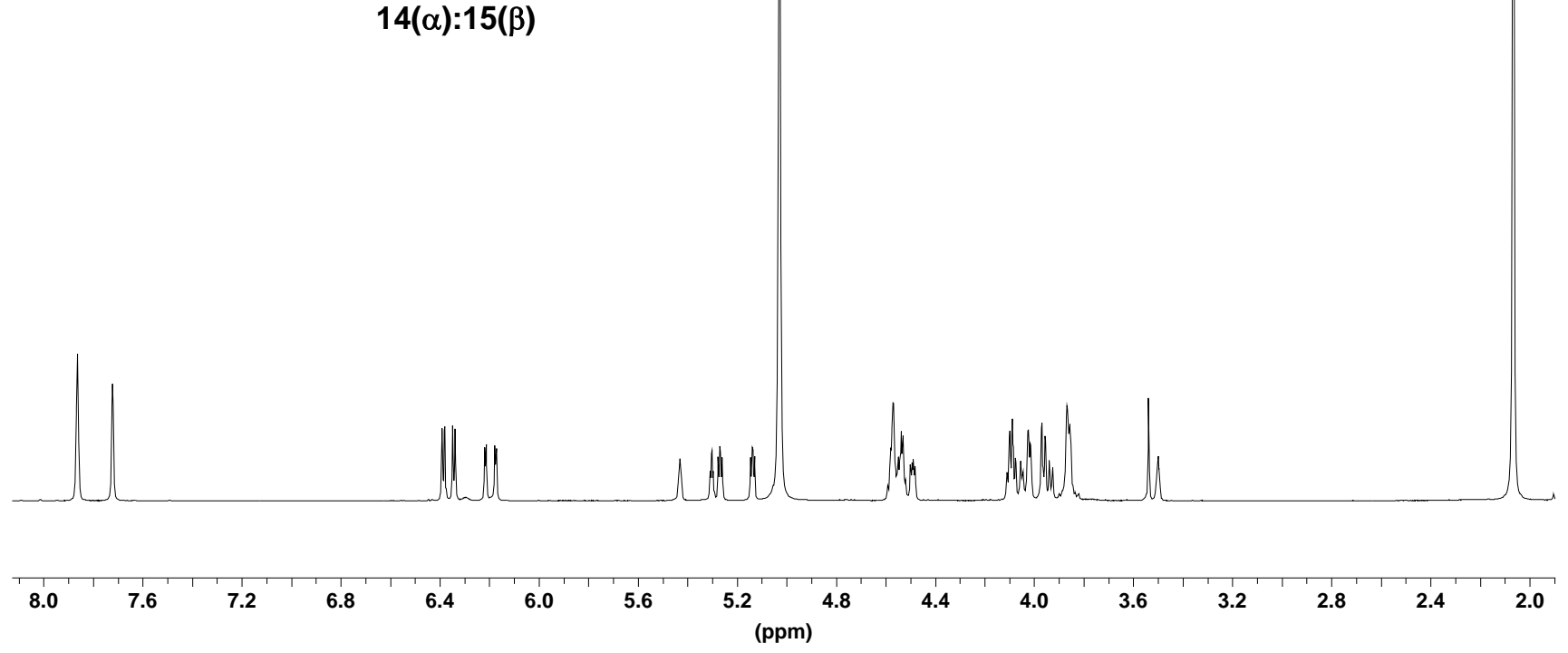




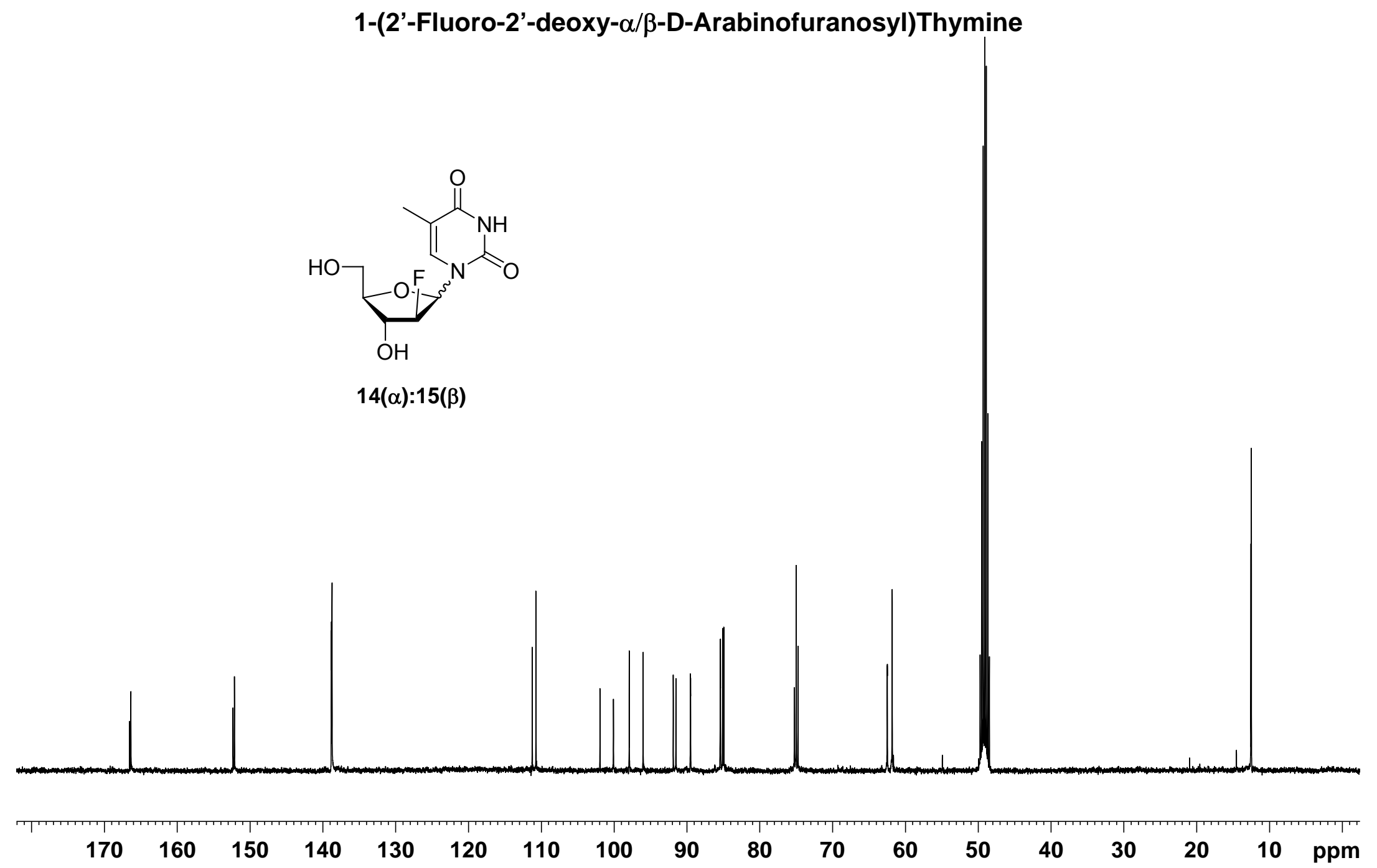




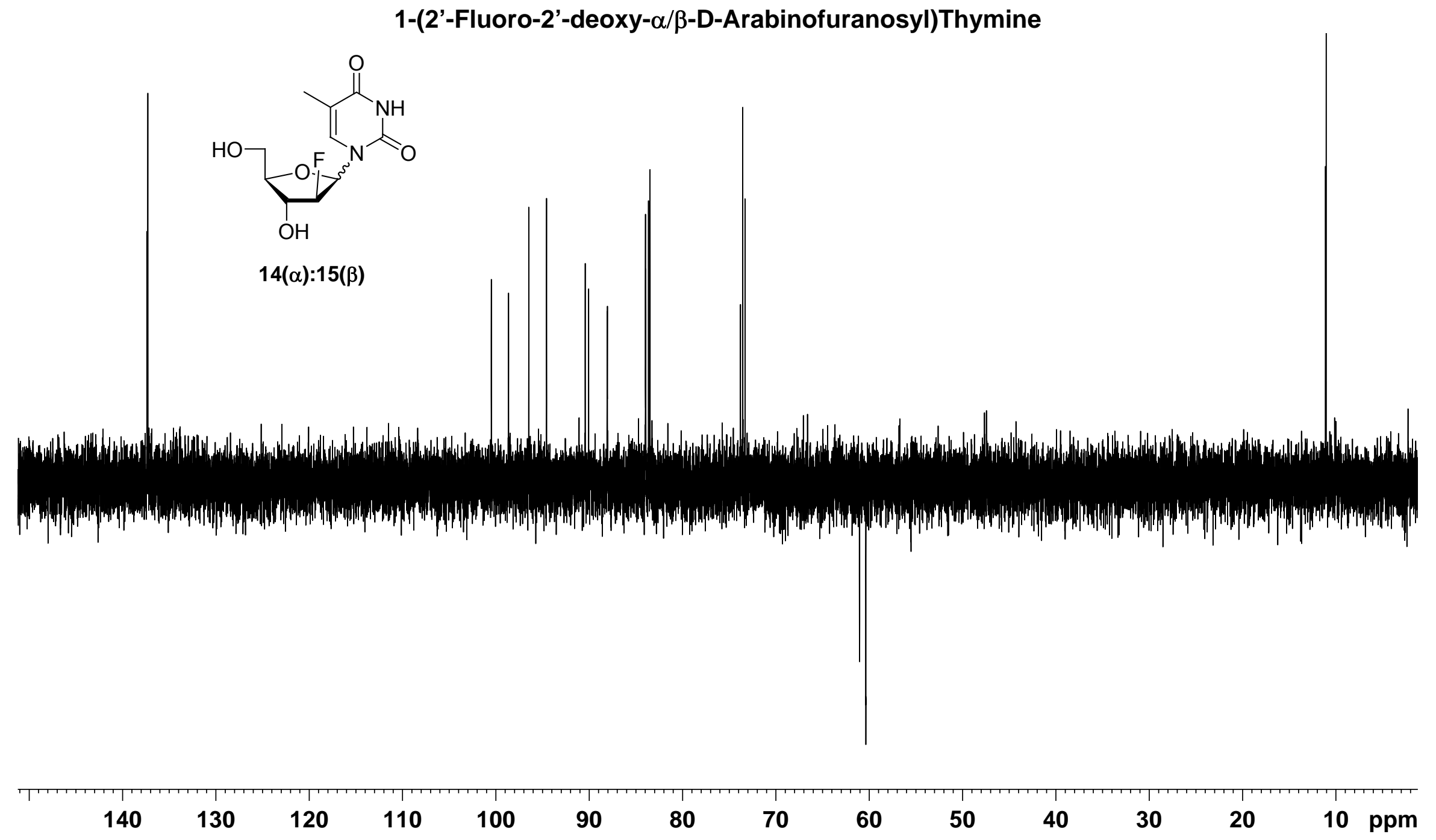




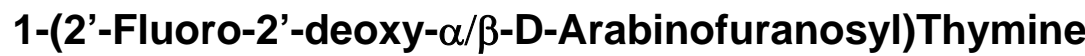

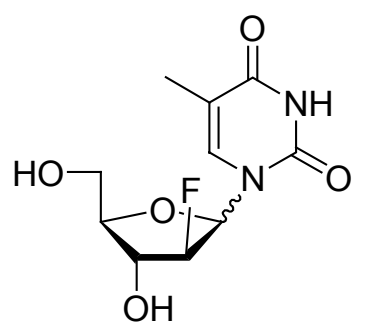

14( $\alpha): 15(\beta)$

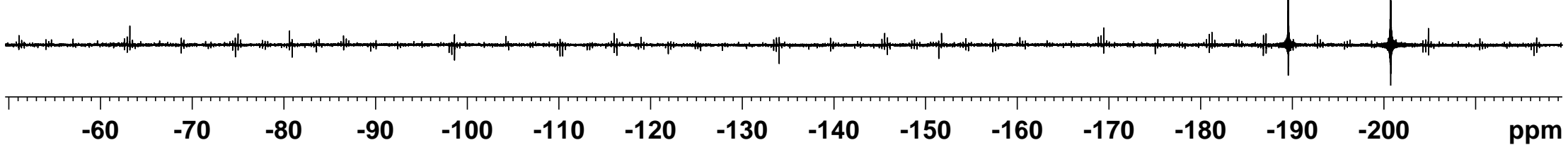


1-(5'-O-Benzoyl-2'-Fluoro-2'-deoxy- $\alpha$-D-Arabinofuranosyl)Thymine

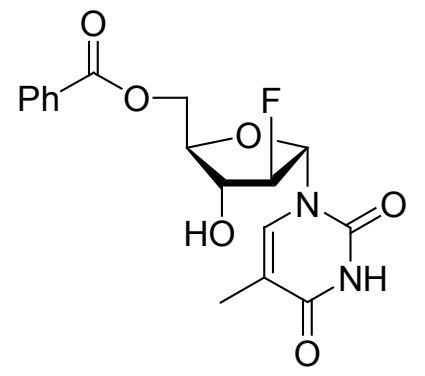

18

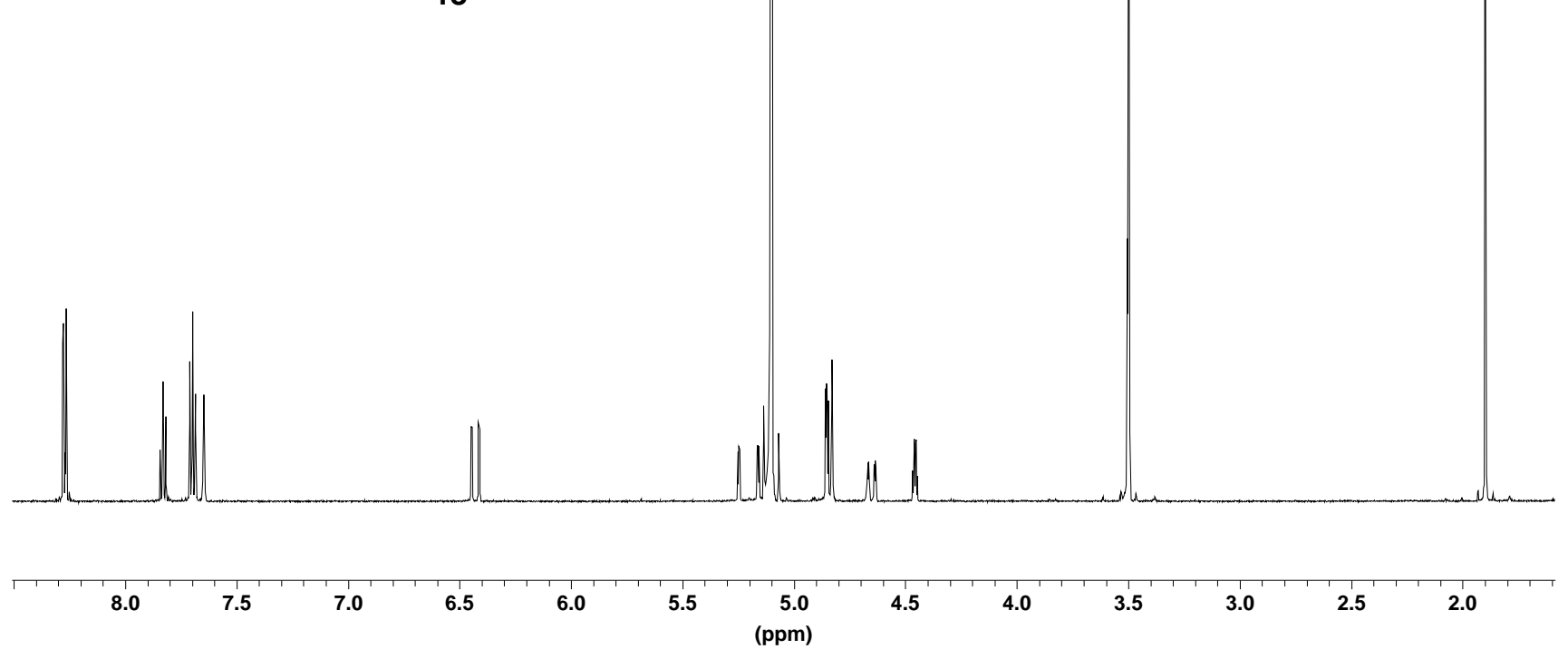




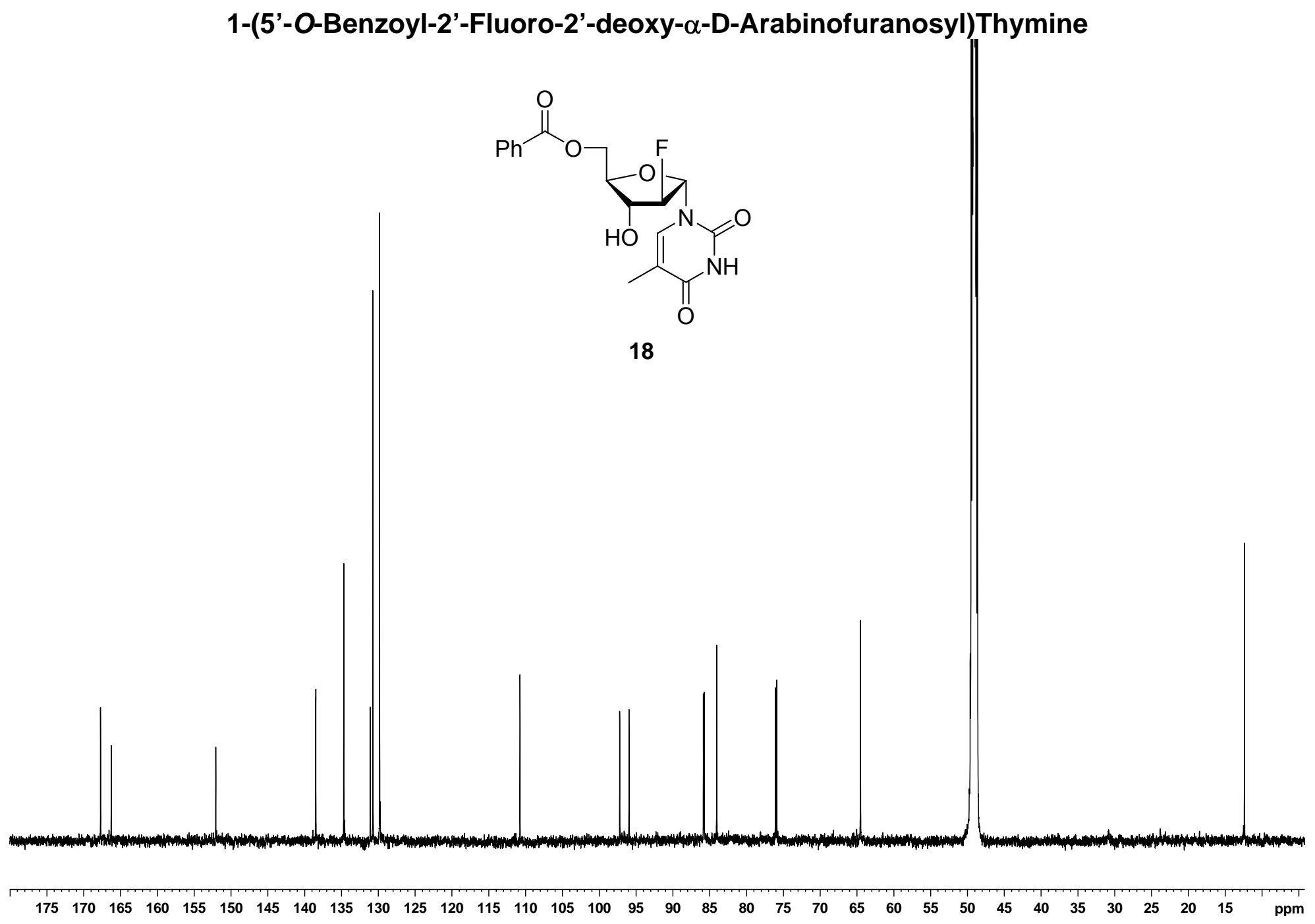




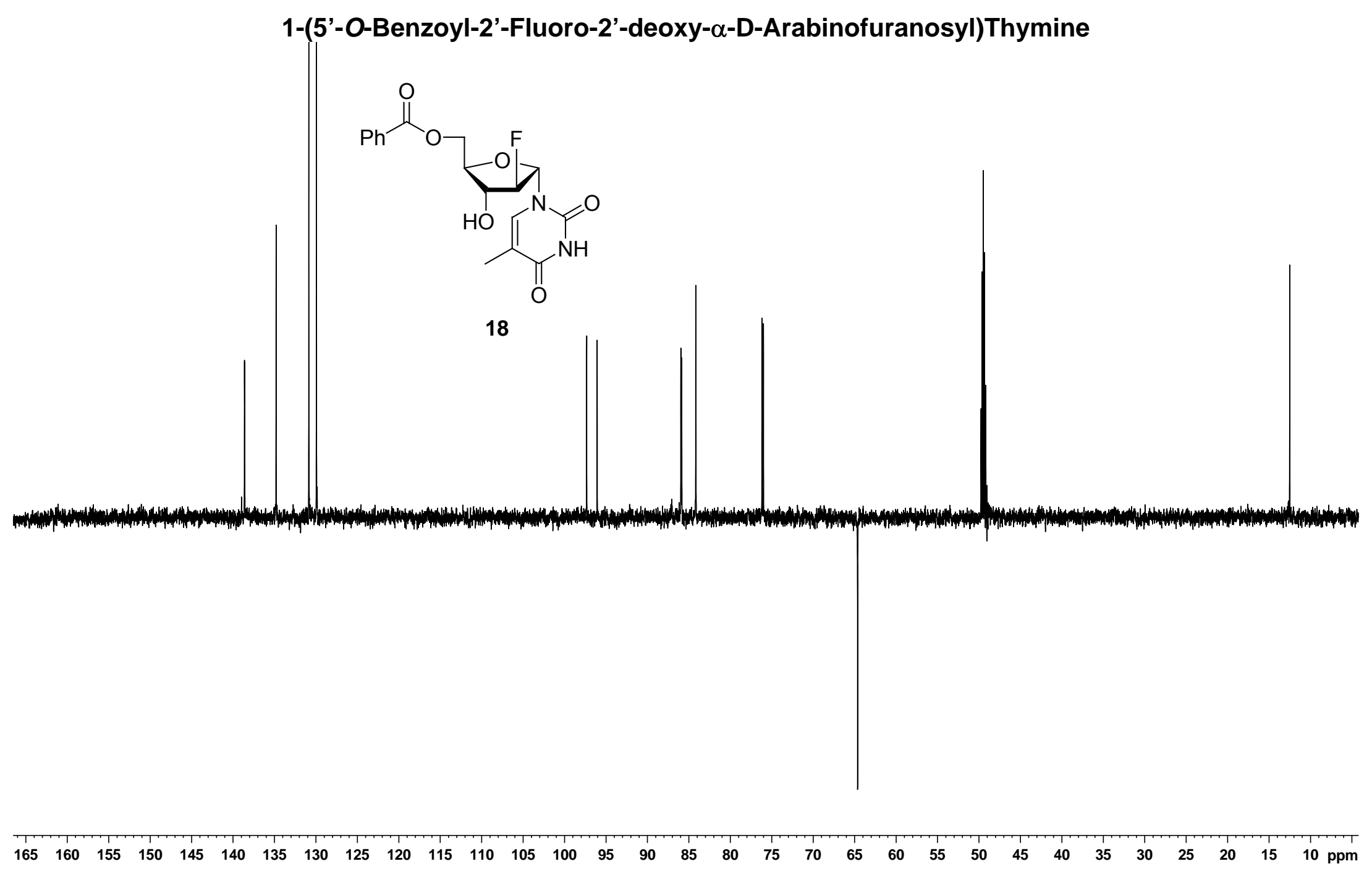


1-(5'-O-Benzoyl-2'-Fluoro-2'-deoxy- $\alpha$-D-Arabinofuranosyl)Thymine

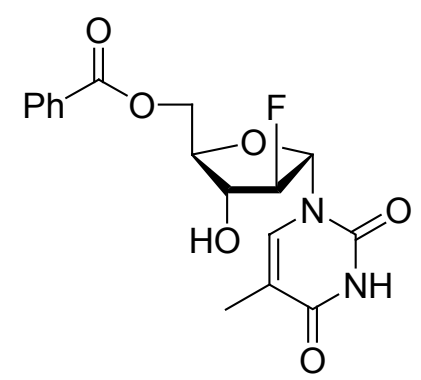

18

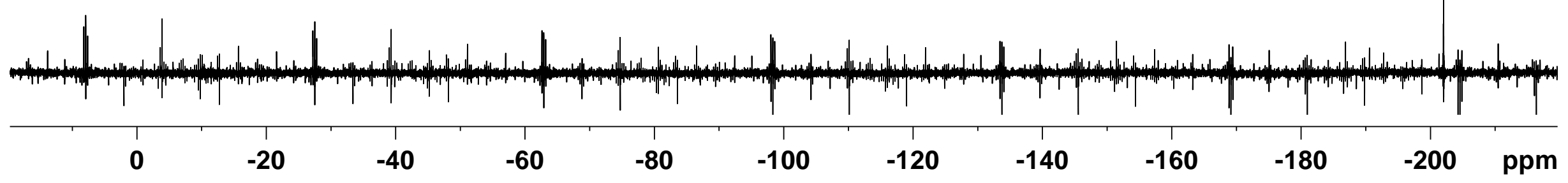




\section{1-(5'-O-Benzoyl-2'-Fluoro-2'-deoxy- $\beta$-D-Arabinofuranosyl)Thymine}

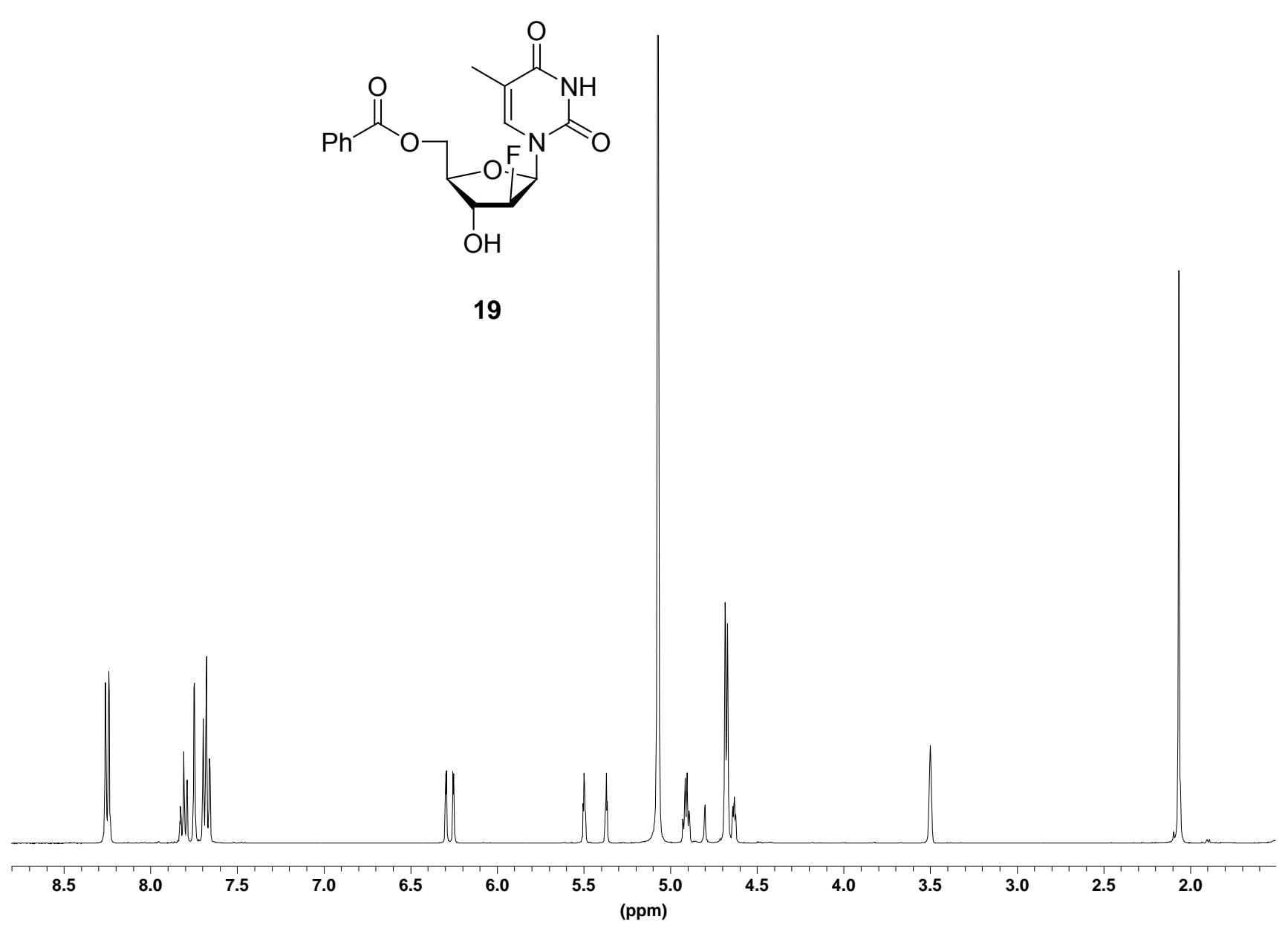




\section{1-(5'-O-Benzoyl-2'-Fluoro-2'-deoxy- $\beta$-D-Arabinofuranosyl)Thymine}

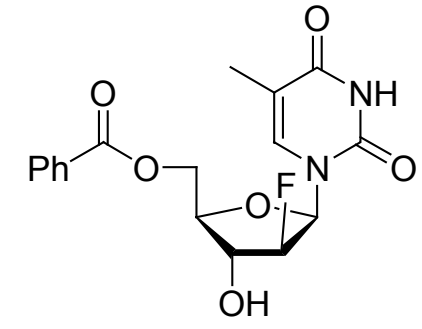

19

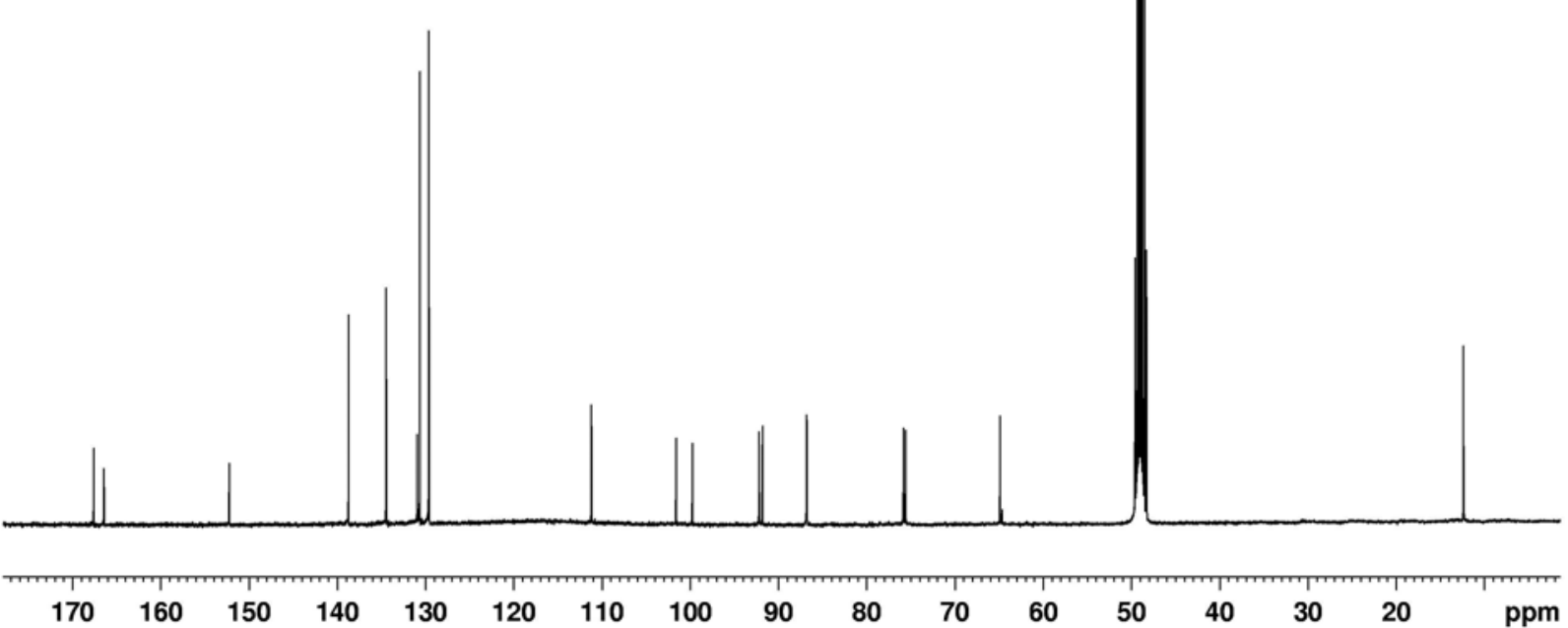




\section{1-(5'-O-Benzoyl-2'-Fluoro-2'-deoxy- $\beta$-D-Arabinofuranosyl)Thymine}

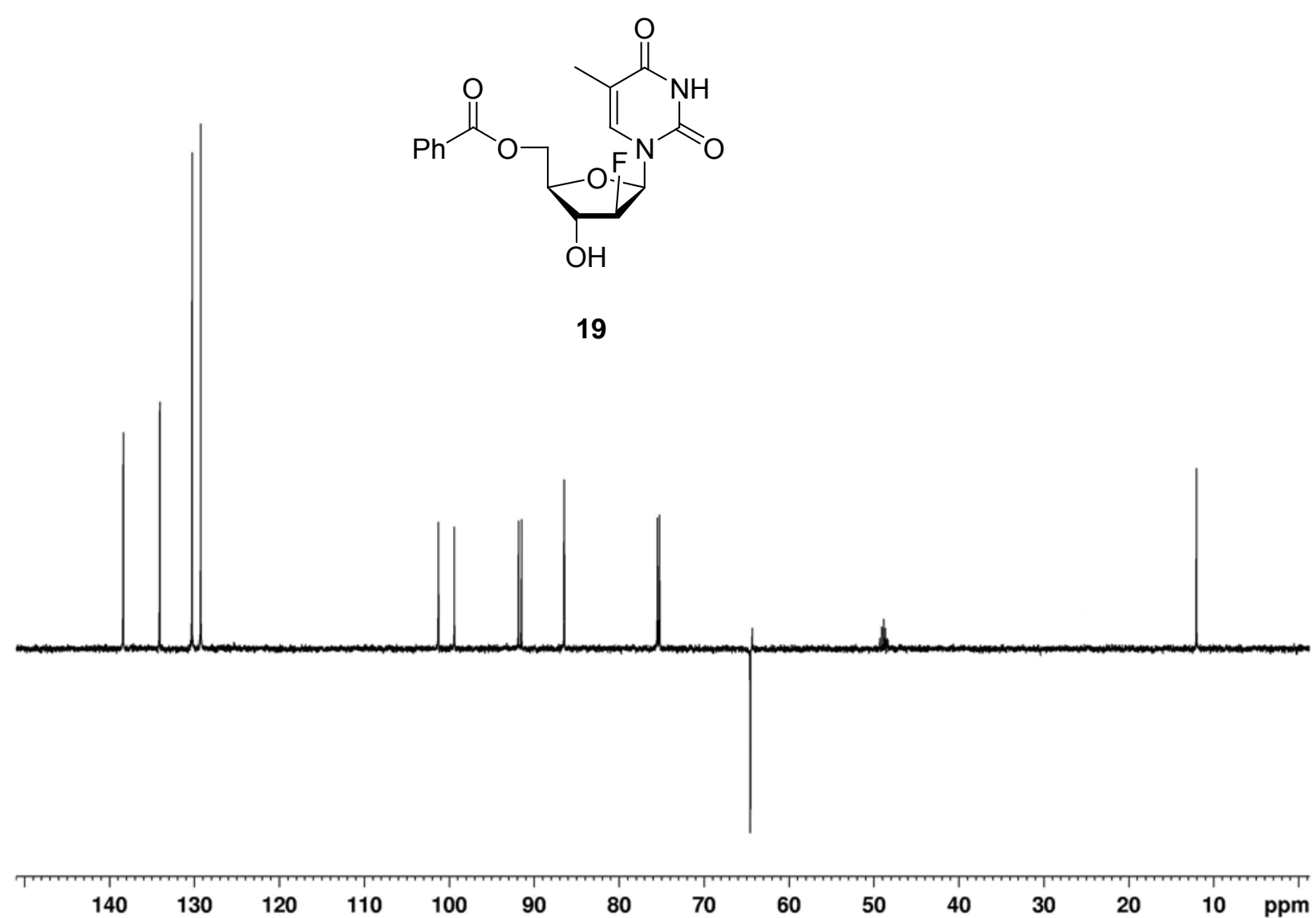




\section{1-(5'-O-Benzoyl-2'-Fluoro-2'-deoxy- $\beta$-D-Arabinofuranosyl)Thymine}

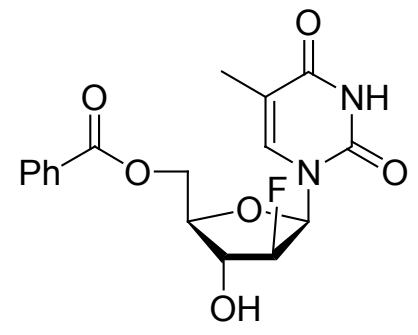

19

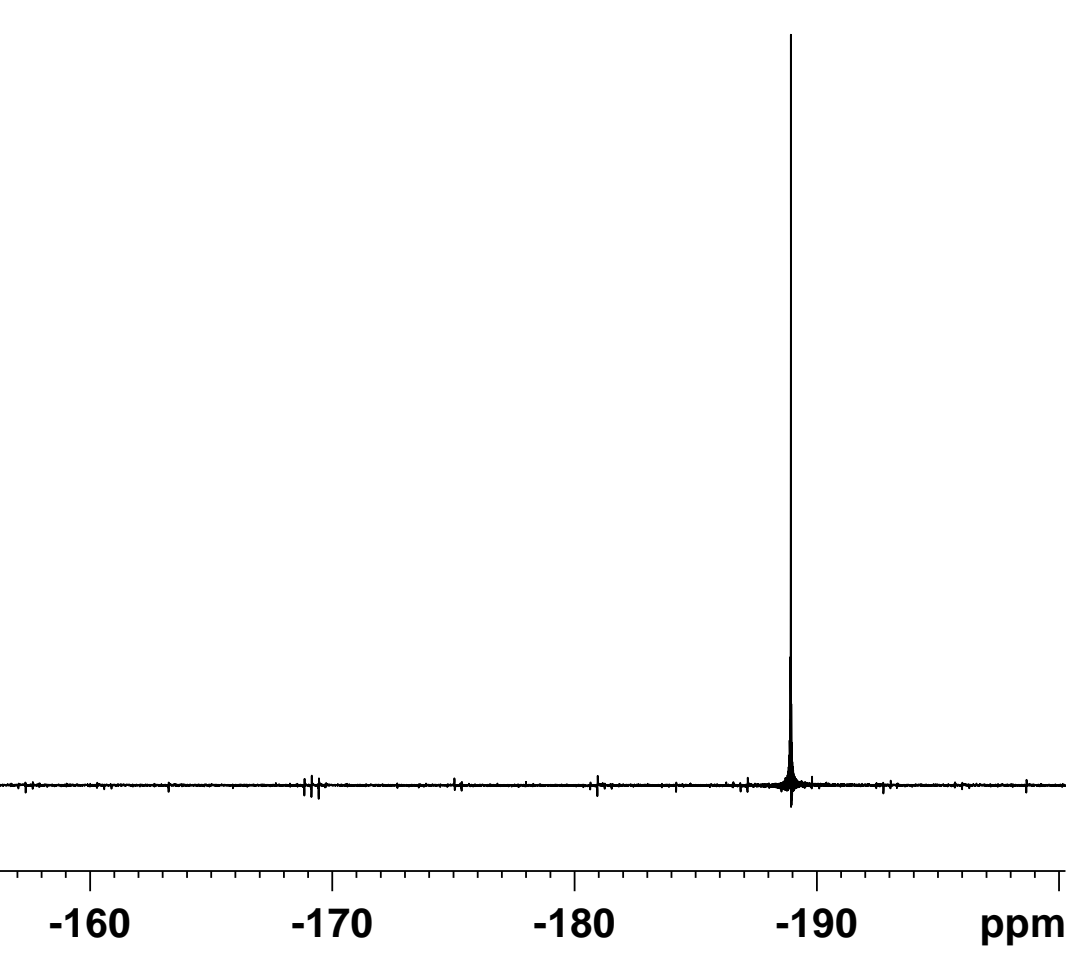

